\title{
L'Omelia di Giacomo di Sarûg sul Battesimo di Costantino \\ Imperatore
}

Edited and Translated by Arthur L. Frothingham 

2 Orientalia Variá

Index.

"4421 Pienret (P)-Le décret trilinque de banope.

14422 La garde (Paul de) (hiv Guiechisches aus ttein Osien

$\checkmark 4423$ Descencet (Ch.) Bas-reliefs assipriens dela Bibl. Vaticang.

$\checkmark 4424$ Ginélinear (C).)-Voyage diu movire égyptien dans le 4425 - maspero (g.) - Htymme aw hil.

" 426 e chrader (Cl.). Oie hamen der hreere in den assy442\% Lagarde (Paulde) Purin Eischen hrschiften.

"4428 Sharrabla (P.) Gugliehro Paimondo honcada.

- 4429 Irochinghain (a.2.)- L'Omelia di Giacomodi Saring sul battesino di Constantino imperatore.

" 400 o stenschneider (M.) hotice suv un ouvrage astronomigue inédit d' Sln Haitham.

- 443 , Stern (D.) Die Oppocalypse des Sophonias aus den trapt. [ischen ribersetzt.

" 432 demm (O. wn)-18 ruchstricke der sahidischen bibelibher-

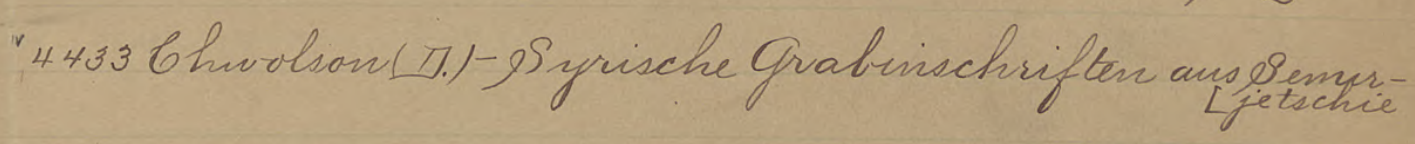

V4404 Lemm (O.ron)-Toptische fragmente gur Patriarchen-

[geschichte alexandrins. 4435 Gullensot (S. B.) Ommaiis- Eicopolis. 
Estratta dall'Arch. Stor. Sic. Nuova serie. Anro III, fase. I, 1878 
REALE ACCADEMIA DEI LINCEI

$$
\text { (ANNO CCLXXIX 1881-82) }
$$

\section{L'OMELIA DI GIACOMO DI SARÛG}

SUL

\section{BATTESIMO DI COSTANTINO IMPERATORE}

PUBBLICATA, TRADOTTA ED ANNOTATA

DA

ARTHUR L. FROTHINGHAM JUs.

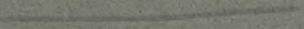

ROM A

COI TIPI DEL SALVIUCCI

1882 


REALE ACCADEMIA DEI LINCEI
(ANNo CCLXXIX 1881-82)

\title{
L'0MELIA DI GIACOMO DI SARÛG
}

SUL

\section{BATTESIMO DI COSTANTINO IMPERATORE}

\author{
PUBBLICATA, TRADOTTA ED ANNOTATA

ARTHUR L. FROTHINGHAM jr.

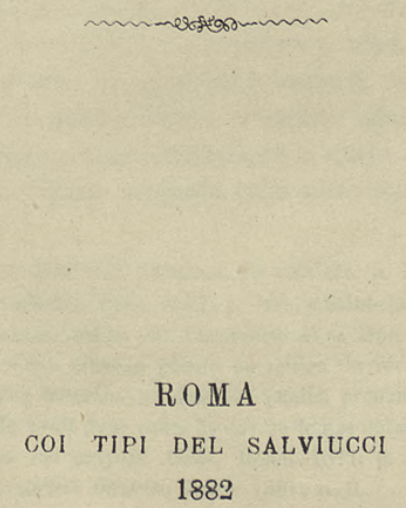




\section{Dी}

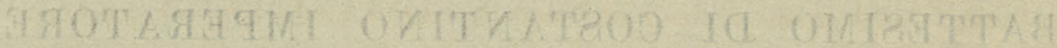

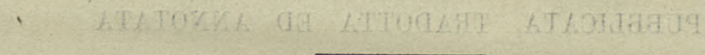

SERIE 3." - Memorie della C'lasse di scienze morali, storiche e filologiche. VoL. VIII. - Seduta del 22 gennaio 1882.

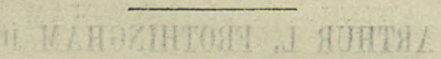


La verità sulla conversione ed il battesimo dell'imperatore Costantino è stata. da molto tempo riconosciuta; avendo la critica moderna spogliato la leggenda del battesimo d'ogni traccia di verosimiglianza, ed essendo tornata alla realtà storica, nel Medio Evo disconosciuta, la quale ci è narrata da Eusebio, Ambrogio, Girolamo ecc. Nondimeno, come studio storico, è assai rilevante il ricercare le origini e lo sviluppo di una leggenda, che ha avuto un' importanza quasi uguale a quella della storia, e anche maggiore se si considera l'abbondanza delle fonti letterarie. Quindi, un nuovo documento di questa leggenda che rimonta quasi al tempo della sua apparizione, dovrebbe certamente considerarsi utile tema di studio: tal è l'Omelia di Giacomo di Sarûg sul battesimo di Costantino imperatore e la sua guarigione dalla lebbra, Omelia che ora presento, per la prima volta, al pubblico.

Giacomo di Sarûg, uno degli autori Siri più fecondi, esercitò una considerevole influenza sopra il suo tempo, ed è uno dei primarî rappresentanti della coltura Siriaca. Egli nacque a Batnê, cittì principale del distretto di Sarûg nella Mesopotamia, nel 452 e morì nel 521 ('), dopo aver tenuto l'episcopato per incirca due anni. Rimandiamo al libro dell'Abbeloos De vita et scriptis Sancti Jacobi Batn. Sar. in Mesop. Episc, etc., ed alle opere ivi citate, coloro che desiderassero conoscere maggiori particolari della sua vita e dei suoi scritti. Dal testo dell'Omelia che ora pubblico apparirà chiaro ch'egli non era, allorchè la scrisse, nei primi tempi della sua attività letteraria; ma di cio ragioneremo a suo luogo. Nè son io il primo a dare importanza a quest'Omelia; recentemente, a varî dotti, come l'ab. Duchesne (') ed il prof. I. Guidi ('), i quali si sono occupati della materia, non è sfuggita la rilevanza di questo scritto del celebre autore Siriaco. Pubblico adunque il testo dell' Omelia di Giacomo di Sarûg, insieme ad una traduzione la quale illustrerò con note dichiarative dei punti ove essa Omelia concorda colle altre fonti della leggenda ed ove

(1) Secondo altri il 29 nov. 522: redi Abbeloos, De vila etc. p. 101.

$\left({ }^{2}\right)$ Écude sur le Liber Pontificalis, Paris 187\%, p. 168. «Saint-Jacques de Sarug, évêque Syrien (452-521) a laissé un discours encore inédit sur Constantin et sa lèpre. Son témoignage est ici d'une grande valeur; il ne s'agit pas d'une allusion glissée au milieu du récit d'autres événements, comme dans Grégoire de Tours: c'est une homélie, un discours public, prononcé officiellement par un personnage considerable. La légende avait donc cours de uis un temps notable dans le pays d'Èdesse», ete.

(') La descrizione di Roma nei geografi Arabi, Roma 1877, p. 44: e La Lettera di Simeone Vescovo di Bêth-Arsâm sopra i marliri Omeriti, Roma 1881, p. 9. 
ne differisce. Credo bene però preporre un esame particolareggiato delle varie fonti e degli scrittori che hanno seguito la leggenda, per mostrare quale ne sia stata l'importanza e la diffusione nelle varie letterature: i documenti ci permetteranno di seguire il suo sviluppo dal $\nabla^{\circ}$ secolo fino al tempo ove fu intieramente adottata, cioè al IX-X secolo. Principierò coll'esame della fonte più antica fornitaci dalle letterature occidentali, gli «Acta Silvestri » : passerò quindi ai monumenti della letteratura latina, la quale mostra testi strettamente collegati agli Atti, cioè gli Apocrifi Simmachiani, il «Liber Pontificalis» etc. Nel capitolo seguente si tratterà delle fonti greche e bizantine, le prime delle quali, Zosimo e Sozomeno, sono d'importanza grandissima, e tale da farci credere, appoggiati come sono da altre ragioni, le quali a suo luogo recherò, che gli Atti ebbero un'origine greca. Difatti, quantunque questi scrittori non arrechino gli stessi incidenti degli Atti, essi fanno fede di un lavorìo che in quel tempo cominciava a farsi nelle menti, intorno alle circostanze della conversione dell'Imperatore. Finalmente ci occuperemo delle letterature orientali, le quali se non ci forniscono testi numerosi pei tempi più tardi, ne hanno di antichissimi, per es. Mosè di Khorene, il quale ci costringe a dare agli Atti un'antichità maggiore di quella che altrimente sembrerebbe probabile. I testi orientali hanno altresì questo pregio, che trovansi meno sotto l'influenza della versione occidentale della leggenda, cioè degli «Acta», e mostrano che in origine essa deve aver avuto piu forme, secondo i paesi ove si sparse. Un'appendice mostrerà come gli Atti siano stati illustrati dalle Belle Arti medioevali, e pubblicamente adottati nelle chiese con iscrizioni antichissime.

\section{Gli Atti di s. Silvestro.}

La storia c'insegna che la conversione dell'Imperatore ed il suo battesimo ebbero luogo a due epoche lontane l'una dall'altra: il primo fatto essendo accaduto incirca al tempo della vittoria sopra Massenzio; il secondo alla fine della sua vita a Nicomedia, allorchè fu battezzato da Eusebio, il vescovo Ariano di quella città ( $\left.{ }^{1}\right)$. Coll'andar del tempo, venerandosi vieppiù la memoria del primo Imperatore Cristiano, gli ortodossi furono verosimilmente spinti a coprire d'un velo, quello che nella loro opinione noceva alla perfezione di lui, la tendenza cioè dei suoi ultimi anni verso alcuni fra i principali Ariani; la prova più manifesta di questa tendenza era senza dubbio l'aver egli ricevuto il battesimo dalle mani d'un Ariano. È dunque probabile che fra loro nascessero facilmente e si spargessero voci le quali sostituivano al fatto storico, a loro spiacevole, una versione più favorevole alle proprie opinioni. Questa consisteva nell'asserire che Costantino, per la sua persecuzione contro i Cristiani, fu afflitto da lebbra, e che questa lebbra fu guarita da Silvestro vescovo di Roma col battesimo.

Ma non bisogna credere che tale sia stata la prima forma che la leggenda abbia presa. Mi sembra certo che la lebbra debbasi spiegare come sviluppo posteriore: la tradizione che vediamo riportata da Zosimo e Sozomeno mostra che erano i delitti, una lebbra per cosi dire morale, la cui guarigione ricercavasi per mezzo

(') Eusebio, Vita di Costantino, lib. 1V, cap. 61. 
del battesimo. Questa contaminazione morale era il risultato di delitti, secondo i testi sopracitati, oppure secondo altri veniva dalla falsa religione, nella quale era nato l'Imperatore, ed era una contaminazione originale: quest'ultima idea vediamo già più sviluppata in Giacomo di Sarûg, ove l'Imperatore è lebbroso fin dalla nascita. Dalla contaminazione morale a quella fisica non havvi che un passo, facile a spiegare dall'analogia di altre leggende, nelle quali cio che prima si diceva metaforicamente passa a senso reale. In questo modo possiamo seguire passo a passo la formazione della leggenda, nelle sue transizioni dalla forma storica alla favolosa in ogni particolare sviluppata. La leggenda infatti nelle sue prime forme attribuisce alla conversione cagioni storiche, vale a dire il voler purificarsi, per esempio, dalla morte di Crispo, ma da questa cosa morale essa passa negli Atti ad una cosa materiale, la lebbra. Quest'ultima forma fu sola adottata presso i latini, mentre i Greci ritennero sempre in parte la prima forma. Dippiu bisogna osservare che gli Atti stessi ne ritengono traccie, poichè dicono che col battesimo furono guarite le macchie del corpo e dell'anima; e qui giova ricordare, come tutti sanno, che la forza purificatrice del sacramento del battesimo si riferisce sempre all' anima, e che in origine ha dovuto, anche nella leggenda, intendersi in questo senso.

Ma veniamo a dire più specialmente degli Atti. La leggenda di Costantino forma parte degli Atti di Papa Silvestro e poche volte, eccetto in codici latini, trovasi separata dal resto della narrazione. Il monumento più antico nelle letterature occidentali essendo gli «Acta », mi è parso conveniente darne qui appresso un compendio (). La prefazione dichiara che Eusebio, narrando le vite dei martiri e dei vescovi di Roma, Alessandria ecc., ha omesso di narrare la vita di s. Silvestro, e che l' autore supplirà a questa mancanza. In alcuni mss. $\left({ }^{2}\right)$ queste frasi sono contorte in modo da far loro dire che gli Atti furono scritti da Eusebio stesso. Silvestro, la cui madre si chiamava Giusta, era presso un prete di nome Cirino. Nel tempo che un tal Timoteo $\left({ }^{3}\right)$ era venuto a Roma da Antiochia durante la persecuzione, Silvestro solo ebbe il coraggio di dargli ospitalità, e quando Timoteo fu martirizzato egli lo seppellì presso s. Paolo. Condotto avanti al prefetto Tarquinio, risponde alle sue minaccie, miracolosamente causando la sua morte. Per questo prodigio venne in grande venerazione presso Cristiani e Pagani. È consacrato diacono

(') Il testo greco leggesi in Combefis \& Illustrium Martyrum lecti triumphi 》 Paris 1659, alla fine del volume sotto il titolo «Sancti Silvestri Rom. Antistitis Acta Antiqua probatiora». Una traduzione latina del testo degli Atti compilato dal Metafraste è data al 31 dicembre nel tom. VI. pp. 1173-118\%, del Surius, De Probatis Sanctorum Historiis.

(') Il titolo del cod. greco della Bibl. Reale di Parigi adoperato dal Combefis e quello del

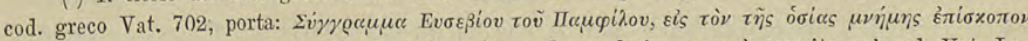

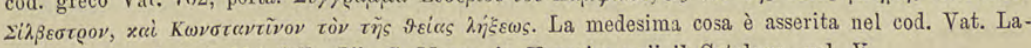
tino 327, e cod. Lat. 116 della Bib. S. Marco, in Venezia: vedi il Catalogo, vol. V.

(") A questo Timoteo si riferisce la notizia di un'antica topografia delle catacombe del VII ${ }^{\circ}$ secolo, come me n' ha avvertito il comm. G. B. de Rossi che l' ha pubblicato (Roma Sott. T. I. p. 141) sotto il nome di opuscolo salisburgense. Essa porta il titolo «De locis scis martyrum que sunt foris civitatis Romae», e vi si legge: «In parte autem australi civitatis juxta viam ostensem paulus apostolus corpore pausat et timotheus episc. et martyr de quo meminit liber Silvestri ibidem dormit ecc. ». Questa menzione degli Atti è di molto pregio ed antichità, 
da papa Milziade ( $\left.{ }^{1}\right)$ alla morte del quale il clero ed il popolo lo giudicarono degno d'essere fatto loro vescovo. Poscia venne a Roma Eufrosino vescovo di Pamfilia, e cagionò varî cambiamenti nella chiesa.

In una caverna del Monte Tarpeo viveva un drago, adorato da maghi ed incantatori, il quale si nutriva di vittime umane. Silvestro cercando distruggerlo, gli viene da s. Pietro comandato di chiudere la porta della caverna con catene. D'allora in poi il mostro non fu più veduto e molti furono convertiti e battezzati a causa di questo miracolo.

In questo tempo comando l'Imperatore di perseguitare i Cristiani e forzarli ad adorare gl'idoli, a ciò consigliato dalla sua moglie Massimiana figlia di Diocleziano. Silvestro cerca rifugio sul monte Syraptim (Soracte). L'Imperatore fu allora percosso di lebbra dalla quale non poterono liberarlo i medici, gl'indovini, e i maghi mandati dalla Persia. I sacerdoti del Tempio Capitolino gli consigliarono allora di lavarsi nel sangue di molti fanciulli, per il qual mezzo guarirebbe. Costantino, uscito per eseguire tale crudele proposta, si commove alle lagrime delle madri ed ai gemiti dei fanciulli, e preferisce la loro salvezza alla propria incerta guarigione. In ricompensa gli apostoli Pietro e Paolo gli appaiono di notte, e gli comandano di mandare per s. Silvestro il quale lo guarirebbe colle acque vivificanti del battesimo. Venuto il Santo gli spiega come coloro che vide in sogno non sono Dei, come egli aveva creduto, ma servi di Cristo, e gli mostra le loro immagini. Impone a Costantino un digiuno e penitenza di sette giorni, durante i quali doveva riparare ai danni da lui inflitti alla Chiesa, e lo fece catecumeno. Terminato il periodo prescritto, lo unse dell'olio santo, e lo guarì di corpo e di mente col battesimo.

Costantino promulga una legge in favore dei Cristiani, e comanda che nel suo palazzo del Laterano sia costruita una chiesa. In quell' anno arrivò il numero dei pagani battezzati a 12,000 senza contare donne e fanciulli. Segue il Concilio radunato nella Basilica Ulpia ove l'Imperatore fa pubblica dichiarazione della sua fede; ed il Concilio promulga varî decreti.

Qui termina la prima parte degli Atti: poscia vi si comincia a narrare la storia dell'imperatrice Elena, la quale a quel tempo favoriva la religione giudaica. Essa stava coi suoi figli Costante e Costanzo in Bitinia, quando sentì che Costantino si era convertito. Istigata dagli ebrei serive al suo figlio che non è Cristo il quale lo ha guarito, ma Dio Padre, colui che adorano gli ebrei. In conseguenza di sio, dopo la risposta di Costantino, vengono gli ebrei a Roma per disputare con s. Silvestro, il quale, dopo aver esposto le dottrine cristiane traendo i suoi argomenti dallo stesso antico Testamento, li converte col risuscitare il toro che Zambri, il più famoso dei dottori Ebrei, aveva ucciso con parole magiche. Del resto è inutile dare un'analisi particolareggiata di questa parte poichè non ha diretta relazione col nostro soggetto.

L'autenticità di questi Atti non può neppure per un momento sostenersi: sono stati considerati come fabbricati in un' età posteriore, da moltissimi scrittori dei secoli passati: la discussione in riguardo di cì fu vivissima nel $\mathrm{XVI}^{\circ}, \mathrm{XVII}^{\circ}$ e

(') Sappiamo, al contrario, che fu fatto diacono da papa Marcellino, per testimonianza di s. Agustino nel libro, De unico Baptismo contra Petilianum, cap. XVI. 
$\mathrm{XVIII}^{\circ}$ secolo, ma ora certamente nessun critico propugnerebbe la loro autenticita. Imperocchè essi stanno in piena contradizione con fatti storici notissimi; e che ciò fosse conosciuto anche a coloro i quali, nei primi tempi dell'apparizione degli Atti in Occidente, li adottarono e li sostennero, ci è provato, fra altre ragioni, dalla confusione nella quale essi cercarono di avviluppare i fatti storici, per fornire una specie di transizione alla leggenda e darle pii verosimiglianza. Immaginarono di attribuire il battesimo di Nicomedia a Costante, figlio del grand'Imperatore: cio viene asserito negli Atti di Liberio, nella vita di Damaso, nella leggenda di s. Felice, e negli Atti di Eusebio. I due primi apocrifi sopra accennati chiamano Costante nipote e non figlio di Costantino il grande, credendo forse di poter farlo credere figlio di Costante, fratello dell'Imperatore. Negli «Acta inventionis sanctae Crucis » vanno un passo avanti e dicono che Costantino fu battezzato da Eusebio vescovo di Roma.

Prima di passar oltre mi è parso utile investigare brevemente alcune cose: la lingua, cioè, nella quale furono originalmente scritti gli «Acta», l'epoca della loro compilazione, ed il tempo in cui furono introdotti a Roma. Questo testo, essendo il più antico delle letterature occidentali, e probabilmente delle orientali, che sostenga il battesimo romano di Costantino, il trovare in quale delle due lingue, greca o latina, fosse compilato, determinerebbe il paese ove ebbe origine la leggenda. È stato creduto che fosse scritto in latino, e di origine romana, ma troppo facilmente; imperocchè quali prove esistono a favore di un tal fatto? non ne sono state addotte verune. Il testo latino finora conoscinto e pubblicato, quello cioè del Surio, è traduzione dal greco del Metafraste: l'antico testo latino non ha veduto la luce. Nei codici greci troviamo due testi, quello antico e di uso generale, cioè il testo pubblicato dal Combefis ('): e un altro che troviamo rare volte ed in codici più moderni, ed è compilazione del Metafraste: del primo abbiamo confrontato tutti i codici che trovansi nella Bib. Vaticana col testo di Combefis pubblicato giusta due Cod. Parigini, ed abbiamo trovato pochissime varianti, e queste generalmente senza importanza e per lo più semplici differenze ortografiche. I codici latini non ci mostrano che un testo solo il quale corrisponde al greco più antico: esso, però, ha varianti importanti che dimostrano l'esistenza di più redazioni: alcuni codici inoltre dicono, nell'introduzione, che gli Atti sono stati tradotti dal greco. Ora noi troviamo un testo antico greco le cui varianti sono pochissime: di fronte a questo, un testo latino che spesso differisce, e che in alcuni codici dicesi espressamente una traduzione dal greco. 亡̀ quindi conclusione naturale che gli Atti siano stati compilati in greco. Se da criterî generali passiamo ad osservazioni particolari, vedremo varî indizî in favore di questa opinione. Come già è stato osservato dall'ab. Duchesne, non si vedono qui, come negli apocrifi di origine romana, copiose indicazioni topografiche: esse mancano quasi intieramente; le poche che vi si trovano mostrano talvolta una conoscenza molto inesatta del luogo: la scelta della basilica Ulpia, p. e., come sede del Concilio, e l'ortografia del monte Syraptim, ecc. Gli incidenti di Timoteo di Antiochia e di Eufrosino di Pamfilia non hanno sapore latino: si noti anche lo sbaglio nell'asserire che Silvestro fu fatto diacono da Milziade. Se

(') Hllustrium_Martyrum lecti triumphi. Paris 1659. 
passiamo alle prove esterne, troviamo che i primi scrittori che alludano agli Atti sono orientali o greci: Mosè di Khorene, Zosimo e Sozomeno. Il primo autore ci dà un compendio esattissimo degli Atti; il secondo contiene indicazioni un poco vaghe, ma tali quali un autore pagano ignorante dei riti della chiesa poteva dare, e le quali sono precisate da Sozomeno, che dice espressamente essere la leggenda del battesimo di Costantino al tempo della sua conversione sparsa fra i Greci. Mi pare dunque, che debbasi ritenere che gli Atti furono scritti in lingua greca. Considererò finalmente a qual tempo siano stati compilati, e quando introdotti in Roma.

Il testo di Mosè di Khorene data probabilmente dal 450 incirca: esso è tanto chiaramente un estratto degli Atti da non poter negarsi che a quel tempo essi erano già divulgati: poichè, se lo storico armeno avesse attinto ad altre sorgenti; ad una tradizione popolare, cioè, la quale avrebbe avuto corso prima della compilazione degli Atti, le sue parole non sarebbero state così chiare, nè concorderebbero in ogni particolare con essi. Poichè vediamo dall'omelia stessa di Giacomo di Sarûg, quanta diversità eravi fra le tradizioni locali d'oriente ed il testo degli Atti. D'altra parte, il passo di Sozomeno ci mostra che al tempo suo tale leggenda era nei suoi principî; egli è contemporaneo a Mosè di Khorene; possiamo perciò fissare con probabilitì la compilazione degli Atti alla prima metà del $V^{\circ}$ secolo.

L'epoca dell'introduzione in Roma degli Atti di s. Silvestro è facile a determinarsi approssimativamente: il decreto attribuito a Papa Gelasio (492-496), se non è da lui stesso promulgato, come alcuni dubitano, è almeno contemporaneo ('): esso parla degli Atti di Silvestro come di un' opera che da alcuni leggevasi a Roma; costume seguito in altre chiese «pro antiquo usu». Queste ultime parole si applicano all' uso fattone in altre chiese ove pubblicamente da molto tempo leggevansi, in opposizione alla lettura privata fattane a Roma, ove, secondo ogni probabilità, giusta l' indicazione del testo, ciò aveva avuto luogo da pochissimo tempo. Di pochi anni posteriori al decreto sono gli apocrifi Simmachiani, i quali mutilano i fatti storici allo scopo di sostenere gli Atti, e mostrano anch'essi di voler abituare i Romani ad accettarli per veri. Quindi possiamo chiaramente dedurre che gli Atti, dopo essere stati da altre chiese adottati, furono in Roma introdotti poco prima del pontificato di Gelasio.

\section{Cenni storici sulla leggenda nella letteratura latina.}

Poco dopo l'introduzione in Roma degli Atti di s. Silvestro, cioè, come ho detto, verso la fine del $\mathrm{V}$ secolo, si formò una letteratura fondata, per così dire, sopra di essi, e, per la maggior parte apocrifa. Ne parla per primo il citato decreto detto di papa Gelasio (494) «De recipiendis et de non recipiendis libris» $\left({ }^{2}\right)$ :

«Item Actus beati Silvestri, apostolicae sedis praesulis, licet ejus qui conscripsit «nomen ignoretur, a multis tamen in urbe Roma catholicis legi cognovimus et pro «antiquo usu multae hoc imitantur Ecclesiae».

Cogli Atti hanno qualche relazione altri scritti come i «Gesta Liberii » $\left(^{3}\right)$,

(') Migne, Patrol. Lat. t. LIX. p. 173-174. - (') Migne, ibid. - (') Coustant, Epist. Romanorum Pontificum. Paris 1721, Append. p. 90. 
gli «Acta Eusebii ( ('), la leggenda di Felice $\left({ }^{2}\right)$, il secondo concilio romano ( $\left.{ }^{3}\right)$, l'Atto di donazione di Costantino (') ece. Tutti questi documenti, come mostrano varì critici e specialmente l'abate Duchesne ( ${ }^{(5)}$, hanno aruto origine durante la famosa controversia fra Lorenzo e Simmaco (498-514), e perciò chiamansi apocrifi Simmachiani. Chi vuol vedere sviluppata con sommo criterio la discussione sopra questa quistione deve consultare il veramente egregio lavoro del Duchesne. Da questi apocrifi ha tratto molti particolari il «Liber Pontificalis » nella sua prima recensione verso il 5140 anche prima.

Esaminiamo brevemente ad uno ad uno gli scritti nominati, premettendo alcune parole sopra un documento quasi contemporaneo agli Atti, cioè gli «Scripta Inventionis Sanctae Crucis» ("), i quali sono enumerati dopo di essi nel decreto di Gelasio in termini più riservati $\left(^{(}\right)$e che li mostrano come di epoca più recente. "Item scri«ptura de inventione crucis dominicae, et alia scriptura de inventione capitis Joannis «Baptistae, novellae quidem relationis: nonnulli eas catholici legunt, sed cum haec « ad catholicorum manus pervenerint, beati apostoli praecedat sententia: Omnia pro«bate, quod bonum est tenete». Questi scritti parlano della visione della Croce etc., ed aggiungono che Costantino fu fatto catecumeno e battezzato da Eusebio vescovo di Roma. Papa Eusebio governò la chiesa per pochi mesi nel 309, ed è chiaro che viene qui in iscena solamente per confusione col suo omonimo Eusebio di Nicomedia $\left({ }^{8}\right)$. Tornando a parlare degli apocrifi Simmachiani troveremo che in istrettissima relazione sono la «Constitutio Silvestri » o Atti del concilio romano, ed il decreto di donazione. Si sosteneva che nel 324 fosse radunato a Roma un concilio generale, detto Concilio II romano i cui Atti furono ben presto riconosciuti come apocrifi. Il Duchesne suppone che siano stati fabbricati verso il 501, per causa della protesta contenutavi in favore dell'antico ciclo pascale, e, naturalmente prima della redazione del Liber Pontificalis. Ecco quanto leggesi riguardo a Costantino nella «Constitutio» nel primo editto: « Eodem tempore cùm multi (nobiles) gauderent quod Constantinus (esset) baptizatus «à Silvestro episcopo urbis Romae, et mundatus fuisset à lepra, per hoc beneficium "quod accepit à Domino Jesu Christo, per Silvestrum episcopum, cœpit integrè «prædicare dominum Jesum Christum, et confiteri ejus beneficia» ( ${ }^{9}$ ). L' Atto di donazione di Costantino a Silvestro è preceduto da un preteso resoconto dato dall'Imperatore del modo in cui fu miracolosamente guarito dalla lebbra per mezzo del battesimo $\left({ }^{10}\right)$. Non è altro se non una mera abbreviazione degli Atti nella parte a cio relativa, e non contiene nulla di singolare.

I « Gesta Liberii » sostengono gli Atti cereando però al medesimo tempo di conciliarli colla storia: vi si legge al principio «Anno regni Constanti regis nepotis Con«stantini magni viri etc. Hoc cùm legisset (Liberius) ex libro antiquo, edoctus à

(') Bolland. t. III, p. 16\%. - (') Non rimane per la leggenda di Papa Felice II, che la notizia del Liber Pontificalis: cfr. Duchesne, Étude sur le Lib. Pont. p. 183-191. - ( (') Coustant, op. citat. append. p. 44, e Sacr. Conc. Labbei t. IX, p. 1579. - (') Sacr. Conc. Labbei t. 1. - ( ( $)$ Op. cit. p. 174-191. ( $\left.{ }^{\circ}\right)$ Bolland. 3 maggio. - (") Migne Patr. Lat. t. LIX, p. 173-174. - (") Un autore della fine del XI. Berengosio, nella sua opera «de Laude el Inventione Sarctae Crucis», cerca di provare che Eusebio in Greco e Silvestro in Latino sono la medesima cosa! - ( $\left.{ }^{\circ}\right)$ Sacros. Conc. Labbei t. I, p. 1579, (10) Cf. ibid. 
«libro Silvestri episcopi Romanorum, eo quod et publicè prædicaret; quia in nomine «Jesu Christi, à lepra mundatum fuisse per Silvestrum Constantinum patruum «Constantis; erat enim Constans non integre Christianus.... Baptizatus autem ab «Eusebio Nicomediensi in Aquilonia villa ». Poscia fa dire da Costante quando parte per la guerra «Sacrilegum Liberium, qui dixit patruum meum fuisse leprosum et «baptizatum à Silvestro episcopo, et mundatum, dum abiero ete. ». Costante avendo riportato vittoria va a Nicomedia ed è ribattezzato da Eusebio abbracciando l'Arianesimo, ed ivi muore. In questo racconto sono stranamente confusi Costante, Costanzio e Costantino.

Gli Atti di Eusebio e la leggenda di Felice rimproverano, come il precedente documento, Costanzio per il battesimo di Nicomedia. La notizia di papa Felice II (Lib. Pont.) dice: «Hic declaravit Constantium filium Constantini haereticum, et « secundo rebaptizatum ab Eusebio Nicomediensi iuxta Nicomediam in Aquilone villa, « et per hoc declaratum eiusdem Constantii Angusti filii Constantini, præcepto mar«tyrio coronatur, et capite truncatur». La prima redazione del Liber Pontificalis è stata attribuita al pontificato di Ormisda (514-523) e probabilmente verso l'anno 514 (') dall'ab. Duchesne: il comm. de Rossi però la crede anteriore di più di 15 anni, e redatta sotto il pontificato di Gelasio. Essa dipende in molti punti dagli apocrifi sopradescritti e parla in questi termini nella vita di s. Silvestro: «Hic in exilio « fuit in montem Syraptim persecutione Constantini concussus, et postmodum rediens « cum gloria, baptizavit Constantinum Augustum, quem curavit Dominus per bapti«smum a lepra. Cujus persecutionem primò fugiens, in exilio fuisse cognoscitur». $\mathrm{Si}$ vede adunque come la leggenda aveva preso radice e si era sviluppata in questi scritti.

Per terminare la serie di citazioni da opere apocrife darò un estratto da una lettera attribuita anticamente a s. Girolamo e scritta ad Eustochio «de vinculis Petri $\left(^{2}\right)$. Essa viene da alcuni considerata come quasi contemporanea di s. Girolamo, ma ciò non è probabile: «Sic quousque ad Magni Constantini imperium ventum est: «qui postquam dignatione superna: per sanctos apostolos Petrum et Paulum visi«tatus, et per Silvestrum Papam baptizatus est: et ex persecutore fidei defensor, «et mandatorum Christi custoditor assiduus factus etc.» Inserirò anche in questo luogo una citazione dal «Libellus de munificentia Constantini», quantunque questo passo sia di età posteriore al resto del documento il quale è creduto essere quasi contemporaneo di Costantino stesso. Vi si parla del Battistero Laterano: «Fontem «sanctum ubi baptizatus est Augustus Constantinus ab eodem Episcopo Silvestro» $\left({ }^{3}\right)$.

Se lasciamo da parte gli scritti apocrifi ed esaminiamo la letteratura latina in generale, non troveremo nessun appoggio per la leggenda nel IV o nel $\mathrm{V}$ secolo. S. Ambrogio «de Funere Theodosii», i padri del concilio di Rimini nel 359 ("), s. Girolamo nella cronica a lui attribuita, Idazio, Prospero di Aquitania ete., forniscono altrettanti testi autorevoli in favore della verità storica. Il primo fra gli storici latini

(4) Duchesne, op. cit. p. 24-28. - (') Opera s. Hieron. Paris 1643, t. IX, Ep. 35. - (') Questo documento fu incorporato dopo la vita di Silvestro nella prima redazione del Lib. Pont. cfr. Duchesne, op. cit. p. 147. - (') Ap. Socrat. 1. II, c. 29; Sozom. 1. IV, c. 18 etc. 
il quale ci dia un testo in appoggio alla leggenda è Gregorio Turonense (c.539 † 595) ( ). Egli compara il battesimo di Clodoveo a quello di Costantino: «Procedit (Chlodovaeus) «novus Constantinus ad lavacrum, deleturus leprae veteris morbum, sordentesque ma« culas gestorum antiquorum recenti latice deleturus:... Erat enim sanctus Re«migius.... sanctitate ita prælatus, ut Silvestri virtutibus aequaretur» $\left({ }^{2}\right)$. Qui vediamo accettato il fatto senza discussione; ma non si deve credere che l'opinionè generale del VI secolo fosse già favorevole alla leggenda: Isidoro di Siviglia (') e Cassiodoro (') non l' hanno adottata, e si può citare per questo secolo in appoggio alla leggenda il solo Gregorio Turonense $\left({ }^{5}\right)$. La ragione di cið è, secondo me, perchè gli apocrifi Simmachiani non esercitarono molta influenza a quel tempo sopra coloro i quali erano lungi dai litigi Romani, che favorivano l'introduzione degli Atti in Roma. Nella letteratura latina non romana, si ritiene percio molto tempo ancora l'antica e storica versione. Or bene Gregorio, come mostrano i suoi scritti. sta moltissimo sotto l'influenza della letteratura ecclesiastica di Roma: la medesima cosa puð dirsi del fautore della leggenda che cronologicamente segue a Gregorio, s. Aldelmo vescovo di Sherborn il quale fiorì nell'ultima metà del VII secolo (†709). Il suo libro « De lau«dibus Virginitatis », contiene, al cap. XII, un passo che tratta del nostro soggetto $\left({ }^{6}\right)$ : "Illud etiam non omittendum reor, in quo præcipuum conservatæ castitatis docu«mentum declaratur: videlicet quod Imperatoris Constantini diuturna valetudo et ele« phantiosa corporis incommoditas, accepto baptismatis sacramento statim ab eodem «salubriter dicto citius curata est, et tam interioris hominis quam exterioris gestus « per cœleste cataplasma ... est sanatus. Præterea absurdum fore suspicabat si cele«berrimum illud spectaculi genus per totos mundi cardines vulgatum .. silentio ... « vilesceret, et a nostris literarum apicibus intactum delitesceret, in quo etc.». « Nam Costantino orbem gubernante ad synodale concilium populis catervatim con« fluentibus duodenos Judæorum præceptores et Pharisæorum Rabbites .... (Silvester) «confutavit etc.». Pare qui che s. Aldelmo parli quasichè la leggenda dovesse sembrare cosa nuova ai suoi lettori, e forse fu egli il primo a tentare di promulgarla nella letteratura di sua nazione - la Britannia. Essendo così interesserebbe di sapere d'onde egli l'abbia tratta; e fortunatamente questo ci è reso facile da un passo per noi importantissimo del Gautberto $\left({ }^{7}\right)$. "Theodorus monachus quidam

(') Hisloria Francorum: ed. And. Duchesne in Hist. Franc. Scr. t. I, p. 28\%, 1. II, c. 31. (`) Ricorderò alcune opere storiche posteriori a Gregorio Turonense, le quali trattando del battesimo di Clodoreo fanno il medesimo confronto con Costantino. La prima di queste opere è anonima e fu scritta verosimilmente verso il 600. È una cronica dei Gesta Francorum fino a Cramno figlio di Clotario: vi si legge « Venit novus Constantinus ad baptismum, abnegatis diaboli pompis ». (And. Duchesne, op. cit. p. 702). Una seconda cronaca anonima creduta contemporanea al re Thierry II $(670-690)$ col regno del quale termina, ripete quasi le medesime parole: «Venit novus Constantinus ad baptismum, « abnegatis diabolo pompisque ejus (op. cit.) 》. Finalmente il Monaco Rorico nei suoi Gesta Francorum (op. cit. p. 807) dice: "Venit (Clodovaeus) ad baptismum quasi novus Constantinus, abnegatis dia«boli pompis». - ( (") Chronicon, in 0p. omn. Roma, 1803, t. VII, pag. 9\%. - (') Historia Tripartita. (') Fortunato, vescovo di Poitiers (530-606) è uno dei primi ad approvare gli atti: nella Vila S. Marcelli (Surius $\mathrm{I}^{\circ}$ Nov. p. 17) egli fa un confronto fra il miracolo operato da quel santo contro un drago e quello di s. Silvestro. - $\left({ }^{\circ}\right)$ Bib. Patrum, Paris t. III, p. 290. - ( $\left.{ }^{7}\right)$ Pertz, Arclicu für ältere deulsche Geschichtskunde, t. X, P. I, p. 333, cf. A. Coen, Di una leggenda relativa alla nascita e alla giovenlù di Costantino Magno, nell'Archivio della Soc. Rom, di Storia Patria, vol. IV, fasc. III, 1881. 
«a Tharso Ciliciæ, atque Adrianus, abbas scholæ Græcorum Romæ quondam positi «simulque græcis ac latinis literis, liberalibus quoque artibus instituti, a papa «Romano, Britanniarum insulæ sunt directi, ac eandem tam salubribus fidei do«cumentis quam etiam sæcularis philosophiæ inlustrarunt disciplinis. Quorum disci«pulatui Aldhelmus quidam vir venerandus inhærens ete.» ('). Questo fatto mentre spiega, come osserva il Coen, la perizia di Aldelmo nel greco e nel latino, ci dà anche il filo d'Ariadne che ei indica in qual modo, sotto le istruzioni di personaggi di origine orientale e greca ma imbevuti delle idee e credenze che a Roma circolavano, egli sia divenuto persuaso della verità della leggenda. Ma oltre questo passo di lui havvi un'altro, d'importanza anche maggiore, nel poema che porta il medesimo titolo "de Laude Virginum »: egli vi dà un compendio degli Atti che, per la sua lunghezza daremo in nota $\left({ }^{2}\right)$. I passi che abbiamo riportato mostrano altresì il modo in cui dal suo centro si propagava poco a poco in ogni paese la leggenda. Quasi contemporaneamente il venerabile Beda ( 7735$)$ compendio gli Atti nel discorso * De - Inventione Sanctae Crucis» $\left({ }^{3}\right)$. Egli ne differisce però in varie particolarita, ore si mostra del tutto originale: dice cioè che Costantino nell' uccidere il drago, dal veleno di quello diviene lebbroso e dopo l'apparizione degli apostoli, l'Imperatore confida a s. Elena la cura di far venire s. Silvestro. Il santo in ricompensa della guarigione fatta ottiene che si cavino da un pozzo i corpi degli apostoli e si collochino in un posto d'onore.

L'epaca alla quale i Sommi Pontefici adottarono la leggenda e gli Atti non ci è dato

(') Teodoro fu nominato circa lanno 668 arcivescovo di Canterbury e mandato dal papa in Britannia accompagnato dal suo amico Adriano. Essi vi causarono un gran rinascimento nella letteratura e nei studi; cfr. Sharon Turner, The History of the Anglo-Saxons vol. III, p. 235 e 236.

(') Hen. Canisii, Lect. Anl. auct. a Jac. Basnage, Ant. 1725, vol. I, p. 723 che riportiamo testualmente. Tempore quo clarus fulsit regnator in orbe Cui Constantinus censebant secula nomen: Sylvester Romana degebat Praesul in urbe Sanctus, Apostolicae qui rexit culmina sedis. Plurima hic fecit virtutum signa sacerdos, Quae modò per mundum chartis inserta leguntur. Denique squamigerum vinxit virtute draconem Fortia ferratis constringens vincla catenis: Qui prius è crypta funesto flamine spirans, Romani regni vexabat jure catervas;

Dum contempserunt Christo famularier uni, Natricis horrendi cultum praestare putantes: Sed cùm strinxisset beluam collaribns arctis, Morbida pestiferi compescens flabra draconis, Civibus exoritur mox exultatio laetis:

Bestia delituit quae pridem saevire solebat, Idcircò statim baptismi gratia fulgens Cœlitus illuxit Romanis arcibus ut sol.

Hic Constantini curavit lurida membra,

Horrida leprosis explodens ulcera squamis:

Dum regnatorem Christi sub gurgite tinxit. Quapropter Roma fregit delubra sacelli Prisca nefandorum contemnens fana Deorum, Et potiora rudis sectatur culmina templi, Qua Salvatoris signata cruore coruscant, Perpetaosqne Dei produnt per secla triumphos. Sic mundum propriis ornabat moribus amplum, Dum regem Romae correxit dogmate Christi. Porrò conflictum gessit didascalus idem Contra bis senos certantes arte magistros, Qui pignus mundi fallaci voce negabant, Dum mediatoris spernebant vota vocantis, Lucida perpetuae qui pandit lumina vitae. Quapropter cunctos fretus coelestibus armis Vicerat extemplò voti certamine compos: Ac velut adversus frendentes ore molussos Frustra qui superas implent latratibus auras, Ferrea scripturae direxit spicula miles: Dum consternata mox omnes terga dederunt Excepto Zambri, qui tunc necromantica fretus, Ignarum populum ecc. ecc.

(") Opera omnia, Colon. Agrip. 1612 tom, VII, p, 356-57, 
di precisare. Deve essere stato però molto prima del tempo di Adriano I (772 a 795). Questo pontefice li cita già come testo autorevole nella sua lettera a Costantino ed Irene, e ne parla nella terza a Carlomagno. La prima di queste lettere fu letta nel 787 al secondo Concilio di Nicea ( $\left.{ }^{1}\right)$. Essa si è dunque conservata in una traduzione greca: adduce gli Atti come antichissima testimonianza a favore del culto delle immagini

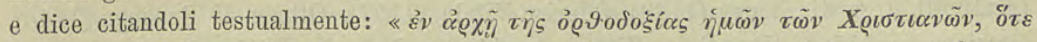

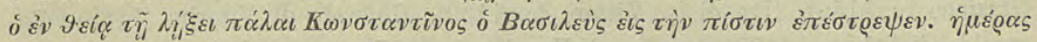

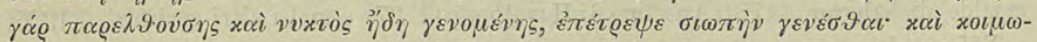

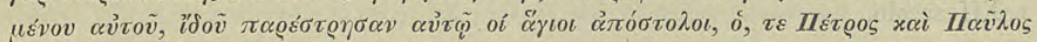

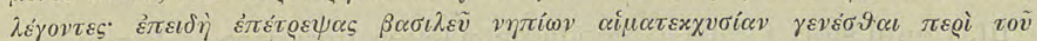

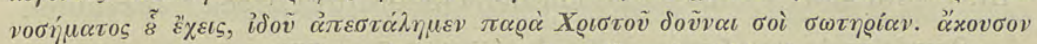

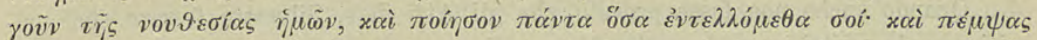

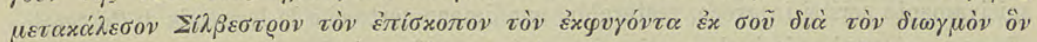

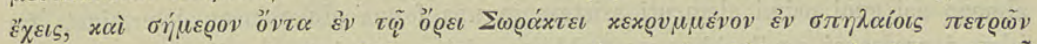

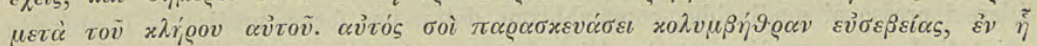

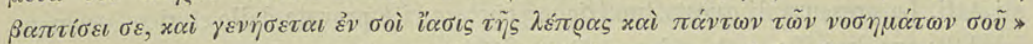

Questa lettera, come noteremo, è di molta importanza ed implica quasi approvazione ufficiale della leggenda; e mentre in Oriente ebbe per risultato il risorgimento di essa, in Occidente le diede maggior impulso: difatti dopo cio non troviamo che pochi seguaci del racconto storico $\left({ }^{2}\right)$, e le citazioni per la leggenda si moltiplicano nel secolo susseguente. Papa Nicolo $I^{\circ}$ (858.867), citando un passo del « constitutum Silvestri $\left(^{3}\right)$, chiama quest'ultimo « Magni Constantini baptizator ». La cronica di Adone di Vienna (c. $850 \mp 875)\left({ }^{*}\right)$ narra che «Constantinus in fide eruditus a B. Silvestro «Papa, fecit miro opere baptisterium Romae, ubi baptizatus est iuxta basilicam «B. Joannis Baptistae, quae appellatur Constantiniana».

Giovanni Diacono, autore delle vite dei vescovi di Napoli (c. 872) ( ${ }^{\circ}$, ha copiato il «Liber Pontificalis» in molti punti, e fra altri nel racconto del battesimo di Costantino, nella vita del vescovo Zosimo. «Hic Sanctus Silvester exilio fuit in «Monte Serapi, et postmodum rediens cum gloria baptizavit Constantinum Augustum «quem curavit Dominus a lepra». Dobbiamo considerare appunto il «Liber Pontificalis » come un elemento importantissimo nella propagazione della leggenda. Presto tenuto in alta considerazione ebbe un'influenza incontrastabile sulla letteratura latina, tanto storica quanto ecclesiastica.

Il famoso Hincmar arcivescovo di Rheims (c. 850 \% 875) nella sua vita di s. Remigio ( $\left.{ }^{(}\right)$ripete le medesime parole già citate di Gregorio Turonense, quando parla del battesimo di Clodoveo. Noteremo in questo luogo che la Storia Tripartita di Anastasio il Bibliotecario (e. 880) compilata da Giorgio Sincello, Niceforo e Teofane, riproduce integralmente le parole di quest'ultimo quando tratta della leggenda nella vita di s. Silvestro ( ${ }^{\text {) }}$ (vedi p. 15), quantunque converrebbe piuttosto citarla nel capitolo sulla letteratura greca.

(') Sacrosanta Concilia, Labbei Venet. 1729. T. VIII, p. 748. - ( $\left.{ }^{2}\right)$ Bisogna però eccettuare Ia cronica di Freculso (c.850) che sembra aver attinto alle più antiche fonti storiche. - (') Hard. V. 154.(") Nella Hist. Christ. di De la Barre, Paris. 1583, f. 496. - ( () Muratori, Rerum Italic. Script. T. I, P. II, p. 293. - $\left({ }^{6}\right)$ Surius, 13 Januar. - $\left({ }^{7}\right)$ Edit. Paris. 1649, p. 25-26. 
Arrivati a questo punto, ove la leggenda, sotto l'azione della letteratura romana ecclesiastica si è fermamente stabilita nella letteratura latina, poco interessa la storia di ricercare quali siano gli autori in particolare che l'abbiano trattata, nè quali piccole variazioni si possano notare nei loro racconti. Chi voglia potrà consultare, fra molti altri scrittori, Papa Leone IX, Bonizone ( $\$ 1089)$, Hermann der Lahme (c. 1050), Ugo di Fleury (1109), Oderico Vitale (1107), Ottone di Frisinga (1146), Graziano (c. 1150), Ugo di s. Vittore (c. 1130), Goffredo di Viterbo (1186), Romoaldo di Salerno (1178), Anselmo di Havelberg (1137) e molti altri scrittori dell' XI e XII secolo.

\section{Cenni storici sulla leggenda nella letteratura greca e bizantina.}

Quando anche non fosse provato che gli Atti di s. Silvestro fossero stati primitivamente scritti in greco, resterebbe nondimeno certo che la leggenda di Costantino almeno nei suoi primi elementi esisteva in una forma più o meno determinata fra i greci presso ai quali ebbe, come abbiamo detto, una forma media fra la storia e gli Atti. La presenza di questa tradizione popolare ci è provata dallo storico Sozomeno,

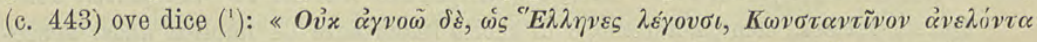

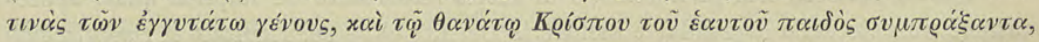

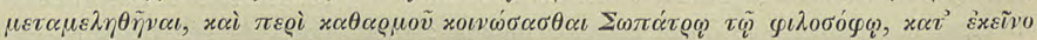

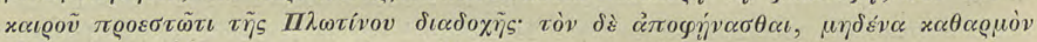

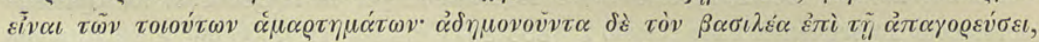

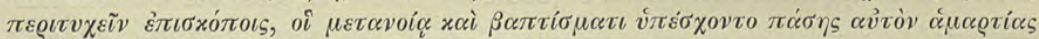

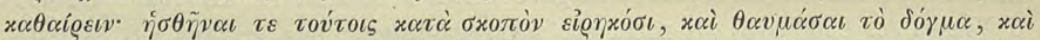

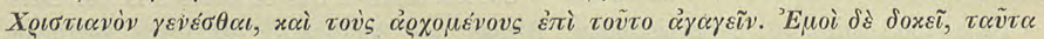

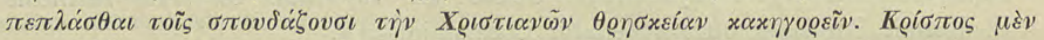

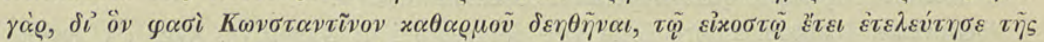

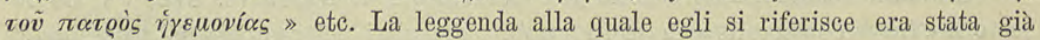
indicata un venti anni prima nella storia di Zosimo $\left({ }^{2}\right)$, ove viene affermato che, cercando invano Costantino di liberarsi dal delitto della morte di Crispo e Fausta, si presenta a lui un certo «Aegyptius » oriundo dalla Spagna e addetto al palazzo imperiale: egli diviene, per le promesse che gli fa, il mezzo della conversione dell'Imperatore. Questa idea di penitenza riviene anche nella nostra omelia di Giacomo di Sarûg ove nel prologo, e in relazione a Costantino, egli dice che la penitenza sarà soggetto principale dei suoi discorsi, e di essa nei versi anteriori dà esempî dal tempo di Adamo, Abraamo, etc. fino ad Ezechia. Si osserverà anche la

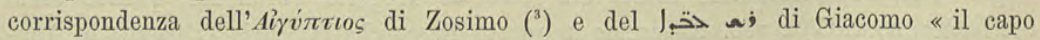
degli schiavi » o sovrintendente del palazzo, i quali furono ambedue gli autori della conversione. Ambedue queste versioni, di Giacomo cioè e di Zosimo, sembrano rimontare ad una medesima sorgente, diversa da quella donde derivano gli Atti, sebbene in Giacomo di Sarûg trovisi uno sviluppo posteriore ove già è adottata parte degli Atti.

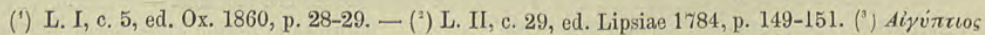
iis हैं 'I 
Pare esservi una lacuna d'un secolo incirca prima di giungere ad uno scrittore il quale accolga la leggenda per vera, ed egli pure era possibilmente sotto l'influenza di letterature orientali. Giovanni Malala di Antiochia, il quale secondo ogni probabilità fiorì nell' ultima metà del $\mathrm{VI}^{\circ}$ secolo la sostiene, nella sua storia ecclesiastica, ove cerca conciliare la leggenda colla storia, parlando prima della visione della croce, e della vittoria sopra Massenzio. Poscia aggiunge $\left(^{1}\right)$ : «Kai v

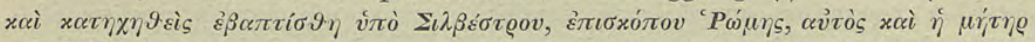

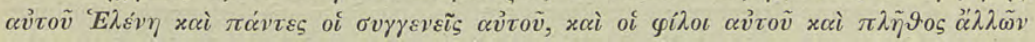

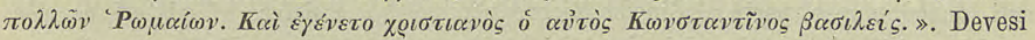
osservare che Malala non parla della lebbra, ma lascia intendere che la conversione fu causata dalla visione celeste e non perchè l'Imperatore cercasse di esser guarito della sua infermita corporale. Egli non pare aver attinto le sue notizie direttamente dagli Atti, ma aver adottato una forma media e quasi di conciliazione, ove concede pressochè tutto alla storia; forma che fu seguita spesso in Oriente invece della Romana. Un altro autore, Leonzio di Gerusalemme, quasi contemporaneo di Malala, nella sua opera contro i Monofisiti $\left({ }^{2}\right)$ cita un passo della disputa cogli Ebrei sostenuta da Silvestro; cosa importante perchè mostra che gli Atti avevano fin d'allora qualche considerazione in Oriente e potevano darsi come autorità.

In appresso siamo forzati di scendere fino alla fine dell'VIII secolo per trovare fautori della leggenda. Questa lacuna si pù forse spiegare per l'antagonismo fra l'Oriente e l'Occidente che impediva di accettare cio che in Roma era ritenuto.

Il risorgere poi della leggenda nella letteratura greca può benissimo attribuirsi, come ho accennato, alla lettera scritta da Papa Adriano I nel 786 per il settimo concilio generale tenuto a Nicea nel 787, quando le relazioni amichevoli furono ristabilite fra le due chiese. Egli diresse questa lettera all' Imperatore Costantino VI, ed alla sua madre Irene; in essa egli difende il culto delle immagini e cita Ie stesse parole degli Atti ove è raccontata l'apparizione degli apostoli all'Imperatore e la presentazione delle Ioro immagini fatta da s. Silvestro. Quasi immediatamente dopo cio troviamo le prime notizie della leggenda presso gli storici bizantini. Teofane (785-818) è il primo a prendere il soggetto in considerazione. Da lui sono narrate ambidue le versioni, mostrando così che non era per dimenticanza del vero fatto che egli favoriva la leggenda. Questa osservazione si applica a tutta la letteratura greca ove si sostiene una forma della leggenda che omette la circostenza della lebbra e dà in suo luogo una causa morale: ma di cio ragioneremo in seguito. Teofane $\left({ }^{3}\right)$ adunque parla dell' apparizione della croce lucente durante la battaglia con Massenzio, d'una visione notturna del Signore: dopo cio, secondo lui, l' Imperatore si mostra sempre zelante per la religione cristiana e distrugge i preti degli Idoli e gli indovini. Ecco le sue parole alla data A. M. 5814, ciò̀ A. D. 314.

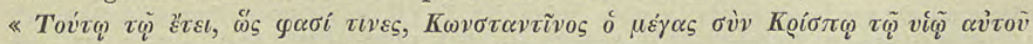

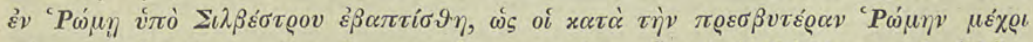

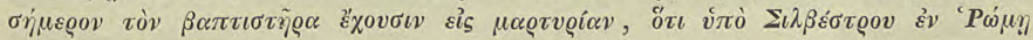

(') Ed. Bonı 1831, p. 316 e 317. Lib. XIII. - ( $\left.{ }^{3}\right)$ Mai, Script. vel. T. VII, p. 134. - ( $\left.{ }^{8}\right)$ Bonn. 1839, T. I, p. 19, 24, 25. 


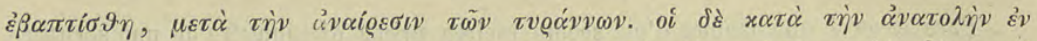

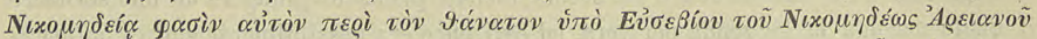

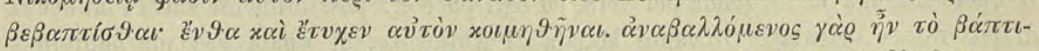

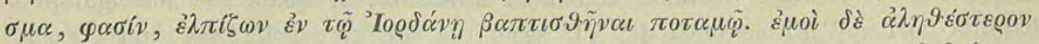

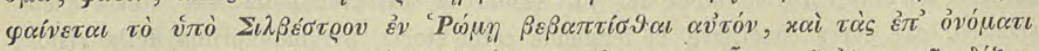

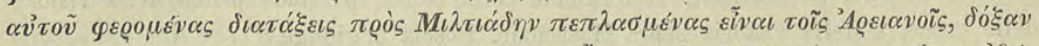

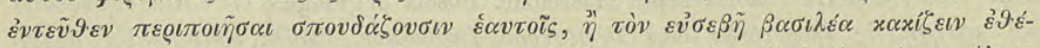

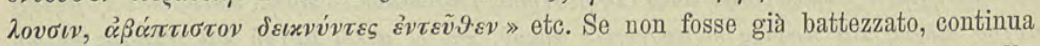
egli, quando prese parte al Sinodo Niceno non poteva assistere ai misteri nè alle preghiere fatte dai santi padri, ciò ch'è «iniquo non solamente di dire ma di sentire». Argomenti ripetuti «ad nauseam» dai fantori del battesimo romano.

Giorgio il Monaco cognominato Hamartolos, il quale porta la sua storia fino all'anno 840, consacra un capitolo intero del 1. IV, ad un compendio degli Atti sotto

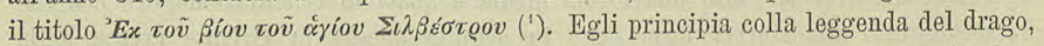
ma omette intieramente quella parte che si riferisce alla conversione ed al battesimo dell'Imperatore. In fine però della vita di Costantino egli discute la questione del battesimo, e dà una testimonianza indiretta in favore di quella Romana: «' $E \mu c-$

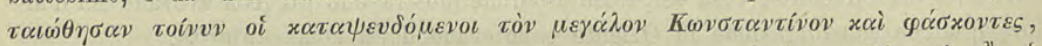

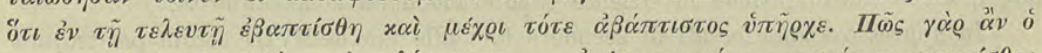

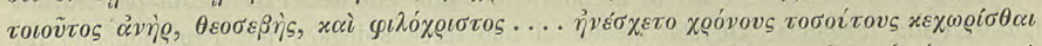

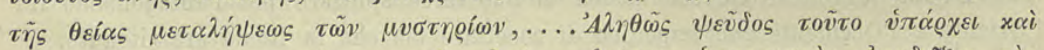

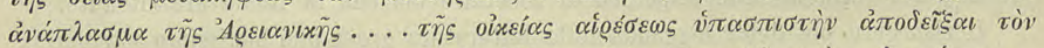

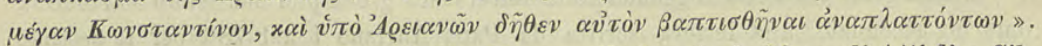

Simeone Metafraste (914-965) $\left({ }^{2}\right)$ nella sua collezione ha inchiuso gli Atti di s. Silvestro, cambiandoli in molti particolari e levandone quelli locali che troviamo nell'antico testo $\left({ }^{3}\right)$.

(') Migne, Patr. Graec. T. C, p. 646. - ( $\left.{ }^{3}\right)$ Surius, De Sanct. Hist. T. VI, p. 1173-1187.

$\left({ }^{s}\right)$ I testi dei Menei e Menologii non si possono rintracciare sicuramente al di là del $\mathrm{X}$ secolo, quantunque forse il loro contenuto sia stato accettato nella chiesa greca in tempi molto anteriori. Il primo Menologio che conosciamo è quello compilato per l'imperatore Basilio II Porfirogenete (975-1025) prima dell'anno 984 *. Ivi leggesi al 2 gennaio, giorno nel quale i greci commemorano

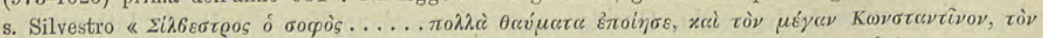

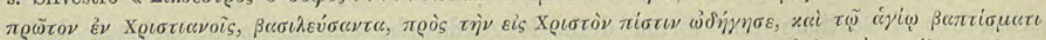

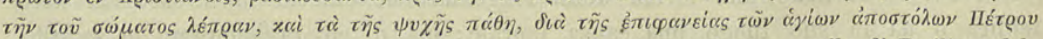
xci I૯ l'XI secolo non ne è che un' abbreviazione nella vita di Silvestro, dicendo: Silvestro convertì Costantino alla fede ed espulse le malattie dell'anima e del corpo per il divin battesimo (Thes. Monum. Eccles. ecc. sive Henrici Canisii Lect. Ant. adjecit Jac. Basnage, T. III, P. I). Aggiungerò qualche parola sopra una vita anonima di Costantino, scritta in greco, che il card. Mai si proponeva di pubblicare ma che per la sua morte è restata inedita. Nel T. VII della Patrum Nova Bibliolheca egli dà, sul frontispizio, il fac-simile del titolo di questo codice (il Greco Vat. 974) e nella P. III, p. 39 dice, in una nota al passo di Bonizone ove egli discute il battesimo di Costantino « De Constantini \& baptismo vetus et notissima quaestio est: nos eius defensoribus, nempe quod Romae a S. Silvestro « eius sacramenti gratiam susceperit, duos nunc adiungimus nempe latinum Bonizonem, et graecum, « quem postea vulgabimus, anonymum magni Constantini biographum ». Ho percorso il detto discorso

- Pubbl, dal card, Albani. Urb. 1727. 
Fra gli storici bizantini dell'XI secolo è Leonzio il grammatico al quale viene attribuita una Cronografia terminata nell'anno 1021. In questa troviamo che « $\boldsymbol{K} \omega \nu$ -

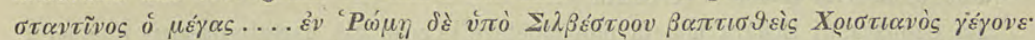

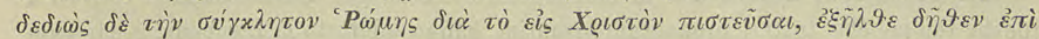

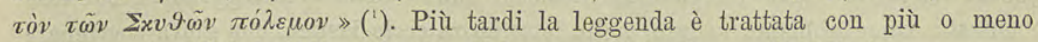
particolari in Michele Glica (1118), Cedreno (1057), Zonara (1118) etc.

Secondo Glica ( $\left.{ }^{2}\right)$ l'Imperatore, apparsagli la eroce vinse Massenzio, ma poscia rallenta il suo zelo; per un miracolo è richiamato dalla sua indifferenza, ma presto pei consigli della sua moglie Fausta torna al culto degli Idoli ed è percosso da Dio colla lebbra - si osservi qui la combinazione cronologica della conversione storica e di quella leggendaria. - I sacerdoti gli consigliarono il bagno di sangue, ma egli avendo misericordia dei fanciulli riceve la visione degli Apostoli, in conseguenza della quale si fa battezzare e guarire da Silvestro. Poscia narra la leggenda di s. Silvestro e del drago, e la sua disputa cogli Ebrei. Finalmente, parlando della morte dell'Imperatore nega il suo battesimo a Nicomedia, asserito, dice egli, da Alessandro il monaco nella storia dell'invenzione della croce: imperocchè l'opinione di chi narra che fu battezzato a Roma da s. Silvestro è più degna di fede; quantunque alcuni Ariani hanno costantemente asserito ch'egli fu da Eusebio di Nicomedia battezzato. In prova di questa sua opinione parla del Concilio di Nicea e del Battistero Costantiniano a Roma.

A Cedreno gli Atti hanno dovuto sembrare di grande importanza, poichè interrompe il corso della sua storia per trascriverli quasi per intero nella parte che si riferisce alla disputa cogli Ebrei. In quella che riguarda Costantino è più breve. A differenza di Glica non parla di successive apostasie come causa della sua lebbra, ma la dà per infermità costituzionale ove parla del suo aspetto e costumi quando

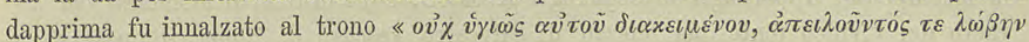

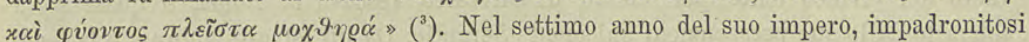

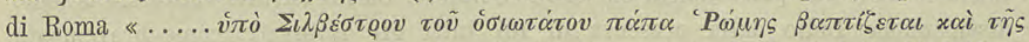

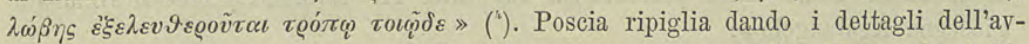
venimento - afferma che furono gli Ebrei i quali consigliarono di uccidere i fanciulli: per il resto non differisce molto da Glica, eccetto la circostanza che menziona esser stato battezzato Crispo insieme al suo padre. Però indicando, com'egli fa, che l' Imperatore era lebbroso fin dalla nascita, si accorda con Giacomo di Sarûg più che gli altri cronografi bizantini. Finalmente incolpa di errore i partigiani del battesimo di

sulla vita di Costantino: esso contiene la narrazione della lebbra e della guarigione dell'Imperatore con quasi le medesime parole degli Atti (in alcuni punti almeno) dai quali fu presa per inserirla, senza legame apparente, nel resto della narrazione; poichè il biografo, avendo narrato la battaglia contro Massenzio, racconta che Costantino, per qual causa non dice, era afflitto di lebbra, la quale non riuscirono a guarire nè medici nè indovini persiani. Segue la proposta uecisione dei fanciulli, l'apparizione degli apostoli, il riconoscimento delle loro immagini fatto dall' Imperatore, la penitenza del medesimo, il suo battesimo ecc. Il codice è stato giudicato del X secolo, ma quando fosse composta la vita stessa è più difficile a determinare.

(') Ed. Boun. p. 84. Questa parte dello scritto è forse una compilazione un poco posteriore a Leone. - (₹) El. Bonn. p. 460,466, 46\%. - ( $\left.{ }^{3}\right)$ El. Bonn. 1837, T. I, p. 473. - (') Op. cit. p. 475. 
Nicomedia: ed a questo punto segue l'uccisione del drago e la disputa cogli Ebrei la quale è inserita quasi « verbatim ».

La Cronaca di Zonara non fa che ripetere le cose già narrate da altri ('); ed a questo tempo che tutti i cronografi bizantini hanno adottato la leggenda, e che essa è entrata, come abbiamo veduto, nella letteratura ecclesiastica, non giova continuare le nostre citazioni nè riferire $\mathrm{i}$ testi di Simeone Logoteta o Metafraste (Cronaca c. 850), Gioele $\left({ }^{2}\right)$, Efraem $\left({ }^{3}\right)$, Niceforo Callista $\left({ }^{4}\right)$ e di altri scrittori posteriori.

\section{Cenni storici sulla leggenda nella letteratura orientale.}

Il testo più antico di data sicura, in qualunque letteratura, che possiamo citare, è la Storia Armena del famoso Mosè di Khorene, il quale fiorì sui primi del quinto secolo. Egli parla in questo modo della conversione di Costantino $\left({ }^{5}\right)$ : « Questi prima «di salire al trono, mentr' era ancor Cesare, fu vinto in guerra. Addormentatosi « con somma tristezza, gli apparve nel sonno una croce di stelle in cielo, intorno a « cui era scritto: Per questo vinci. Fattosi di questa il suo vessillo, e fattolo pre« cedere, vinse la battaglia. Ma poscia istigatovi da sua moglie Massimiana, figlia « di Diocleziano, perseguitò la chiesa. Martirizzatine molti, fu egli per la sua te« merità infetto in tutto il suo corpo da lebbra elefantina. Da questa non poterono « guarirlo gli stregoni ariocesi nè i medici marsi. Percio mando a dire a Tiridate * che gli spedisse indovini dalla Persia e dall'India; ma neppur questi gli recarono "giovamento. Alcuni sacerdoti pagani per suggerimento dei demoni gli comanda« rono di uccidere moltissimi fanciulli in una vasca e di lavarsi col loro sangue caldo « e sarebbe guarito. Egli, all'udire il vagito dei fanciulli e gli urli delle madri, «mosso da compassione, preferi la loro vita alla sua salute. Del che ricevette da Dio "la ricompensa. Apparitigli in sogno gli Apostoli, ebbe ordine di mondarsi col la"vacro del vivifico fonte per mezzo di Silvestro vescovo di Roma, il quale s'era « sottratto dalle sue persecuzioni sul monte Seraptio. Da lui istruito abbraccio la fede, « dissipandone Iddio dalla presenza di lui tutti gli avversarî: come brevemente «t'insegna Agatangelo ».

Questo è uno dei compendî più completi di questa parte degli Atti coi quali concorda in tutti i particolari, eccetto il racconto della visione della croce tolto da Eusebio, e della parte che prende Tiridate nel mandare gli indovini. Bisogna notare che in questo racconto è menzionata una prima conversione dell'Imperatore accaduta per la visione, cosa troppo generalmente creduta a quel tempo per potersi negare. Quindi bisognava supporre, come egli fa, una vera apostasia in Costantino per ispiegare la lebbra; questo è dunque già un tentativo di conciliare la leggenda colla storia. L'importanza del passo per il soggetto è capitale; non è una frase detta alla sfuggita, ma un racconto completo e dettagliato.

Tanto è che fondandosi su cio il chiarño ab. Duchesne ha voluto dedurne l'origine Armena della leggenda; quale sia la verosimiglianza di questa opinione, i lettori possono giudicare. Mosè di Khorene si era veramente ellenizzato in modo da fare sua propria quasi tutta la letteratura greca tanto classica quanto cristiana, della

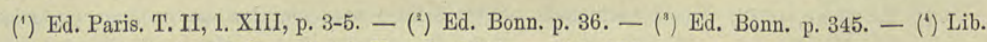
VII, c. $45 .-\left(^{s}\right)$ Trad. del Cappelletti. Venezia 1841, p. 180, 181. 
quale egli tradusse in armeno molte opere $\left(^{1}\right)$ : ogni probabilità ci conduce-a pensare che in questo come in tante altre parti della sua storia fece uso di materiali trovati nella letteratura greca, ed abbia appunto consultato gli Atti di s. Silvestro.

Oltre Mosè di Khorene non ho trovato nell'antica letteratura armena altra traccia della leggenda: dovremo quindi passare ai Siri per trovare prove ulteriori. La più antica di queste è appunto l'omelia di Giacomo di Sarûg, scritta parecchi anni dopo il 473 , e probabilmente più antica che la maggior parte degli apocrifi romani. Questa omelia ci dà una versione intieramente diversa da qualunque altra. La personalità di s. Silvestro, tanto spiccante nelle letterature greca e latina è qui tralasciata. Quando ha luogo l'atto del battesimo si parla semplicemente di un vescovo (aمas) Si suppone che l'azione ha luogo a Roma, quantunque il nome di essa, e qualunque altra indicazione topografica manchi. Tutti i personaggi ad eccezione di Costantino ritengono l'anonimo. Qui dunque mancano tutti i preliminari che riguardano s. Silvestro, la sua persecuzione e ritorno, ete. Costantino è lebbroso fin dalla nascita: la madre è cristiana, ma il padre è ancora pagano: questi cerca in vano la guarigione del suo figlio per mezzo di tutti i medici del suo dominio. Morto il padre, Costantino succedutogli vede l'avversione che la sua malattia cagionava a tutti della sua corte, e manda inviati in tutti i paesi a cercare medici o indovini per guarirlo. Gli indovini babilonesi danno la promessa di guarirlo: vengono, e consigliati da Satana e l'Errore di cui egli è figlio comandano il bagno nel sangue dei fanciulli. A questo si oppongono il capo degli schiavi che è un cristiano, e la madre dell'Imperatore; ma invano, finchè un angelo mandato dal Signore determina l'Imperatore ad accettare la proposta del capo degli schiavi, cioè di farsi guarire per mezzo del battesimo. Il vescovo reso consapevole della decisione dell'Imperatore, si avanza a riceverlo, lo unge coll'olio santo, poscia lo battezza e gli amministra i santi sacramenti. Guarito e convertito, l'Imperatore innalzo lo schiavo alla seconda dignità nel regno, e costui condanna ad essere bruciati l'indovini ed i Caldei. Poscia il tempio pagano ove soleva l'Imperatore adorare gli Dei viene consacrato al culto cristiano. Le grandi differenze di questa versione sono evidenti; ma è impossibile di sapere quante ne furono introdotte per capriccio dello scrittore, e quante possano doversi ad una versione locale della leggenda diversa da quella occidentale. Dobbiamo credere però che i tratti principali, come la lebbra di Costantino fin dalla nascita, la grande importanza data al capo degli schiavi ecc., fossero fondati sopra una tradizione anteriore.

Ma oltre Giacomo di Sarûg troviamo che gli Atti di s. Silvestro furono presto tradotti in siriaco, non più tardi cioè del VI secolo e forse prima: ed essendosi conservati in un manoscritto di quel medesimo secolo sono importanti pel confronto coi testi greci e latini pei quali non abbiamo codici di tale antichità. Sono stati publicati dal Land nel tom. III (p. 46-76) dei suoi « Anecdota » nella storia miscellanea del Zaccaria $\left({ }^{2}\right)$, ma secondo lui non appartengono a quella parte la quale

(1) Cfr. Langlois, Collection des Historiens Anc. el Mod. de l'Arménie. T. I, p. 385-398.

$\left({ }^{2}\right)$ Zaccaria retore, dell' isola di Mitilene, scrisse la sua storia in greco verso il 540, ma essa non ci è rimasta che nella versione siriaca. Egli era creduto da alcuni essere stato vescovo di Melitene o Malatia nella piccola Armenia, ma questa opinione è stata confutata dal Land nell'opera Joannes Bischof van Ephesos pp. 35-37; cfr. Anecd. Syr. T. III, p. VII e segg. 


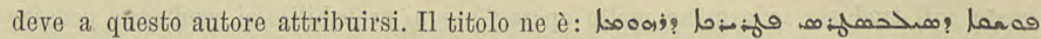

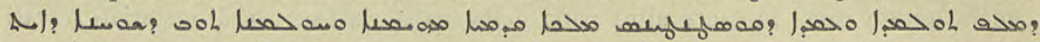

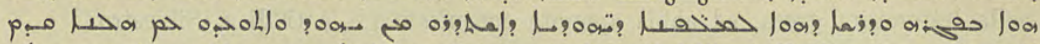

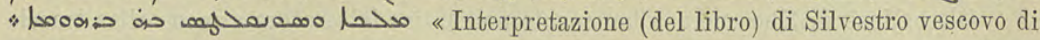
« Roma, che informa della conversione e del battesimo di Costantino primo re fedele, « della guarigione anche dalla piaga del suo corpo, della disputa coi dottori ebrei «mandati dalla Giudea i quali insieme ad Elena si radunarono avanti al re ed al «senato di Roma». Questa traduzione fu fatta sul greco, e cio mostra quanto sia antica la redazione greca. Essendo poi versione letterale, non havvi necessita darne estratti. Il Land però ha trascurato un'altra versione siriaca degli Atti contenuta nel cod. Add. 12. 174 del Brit. Mus. Ne devo la comunicazione all'estrema bontà e cortesia del prof. Wright. Essa non è completa tralasciando tutta l'ultima parte ove viene narrata la disputa di s. Silvestro coi dottori ebrei. Il titolo ne è : ooL |

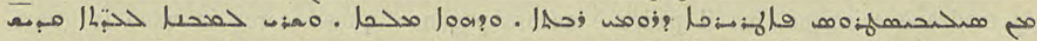
"Segue la storia del grande imperatore Costantino, fedele e vittorioso; come divenne degno di ricevere il santo battesimo da Silvestro papa della gran Roma, e come fu fatto imperatore e comincio ad edificare chiese sante in ogni luogo». Questa versione è del tutto indipendente da quella pubblicata dal Land, ma mostra anch'essa di essere stata fatta sul greco: a parola non si corrispondono, ma non differiscono in quanto al senso; si può affermare, pero, che quella del cod. 12.174 sia alquanto più diffusa e completa ('). Sembrerebbe che la prima forma della leggenda, che correva in Siria e Mesopotamia nella seconda metà del quinto secolo fosse fondata sopra una tradizione più locale e propria degli orientali, ove era omessa quella parte che si riferisce a Roma e al Pontefice, ai tratti infine che la connettono coll'Occidente. Ma l'introduzione degli Atti in Oriente modifico la tradizione locale, e gli scrittori siri posteriori seguono in gran parte questa degli « Acta».

Il primo a mostrare questa influenza è lo storico Dionisio di Tellmahrê il quale fiori verso la fine dell' VIII secolo e sembra aver unito e cercato di concordare la tradizione locale con quella degli Atti; poichè egli narra che $\left({ }^{2}\right):$ « Costanzo « il vecchio e Elena la sua moglie erano fedeli e veneravano Iddio: ed in tutto il * corso del loro regno non fuvvi nessuna persecuzione .......... Costantino però suo « figlio non fu educato nella fede del suo padre, ma di continuo si mostrò perse« cutore della fede cristiana. Anche dopo che una piaga maligna attaccò il suo corpo « non cessò dalla violenza della persecuzione, rendendosi simile all' inferno perse« cutore per il suo zelo nell' idolatria. Mentre era così pieno di minaccie e morte « contro tutti i cristiani, imprigionando, mettendo a morte, crocifiggendo e lapidando, « la piaga della sua persona crebbe, ed una lebbra coprì tutto il corpo. Un odore « infetto come d'un sepolcro pieno di cadaveri la cui esalazione si propaga da ogni «parte: così era egli. Un odore di corruzione da lui si spargeva ovunque. Per « questa ragione incuteva minaccie e morte contro tutti coloro che non adoravano

(') Mi riservo di pubblicare in altra occasione questo testo importante.

(') Dionysii Thelmahharensis Chronici Lib. prim. ed. O. F. Tullberg. Upsal 1848, p. 187 a 191. 
* gli Dei, affinchè essi dessero guarigione al suo corpo. Egli però vedendo che « ogni giorno si faceva più putrido e corrotto il suo corpo per, la lebbra, mandò " a radunare tutti gli indovini, affascinatori, caldei, •

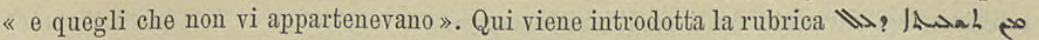
b istratto) dalla storia del Gran Costantino» e prosegue l'autore dicendo : «Poichè era coperto dalla lebbra lo consigliarono i suoi medici di radunare « e far venire trecento fanciulli per veciderli e lavarsi nel loro sangue ». Del resto non havvi bisogno di riferire il rimanente del suo discorso poichè non fa che abbreviare gli Atti, narrando la pietà di Costantino pei fanciulli, l'apparizione di Pietro e Paolo, come fu cercato s. Silvestro il quale si era rifugiato nella caverna di un monte, le immagini degli apostoli, la penitenza dell' Imperatore per sette giorni, la sua guarigione e confessione di fede, la costruzione della chiesa Costantiniana, ch' egli per equiroco chiama una chiesa in Costantinopoli. Questi fatti, secondo Dionigi, ebbero luogo nel nono anno del regno di Costantino.

Dopo questo serittore ci mancano altre citazioni nelle letterature orientali per molti secoli : poichè dopo Dionisio non fuvvi per molto tempo nessuno storico siro che trattasse quel periodo.

La letteratura cristiana di Egitto non sembra aver adottato affatto la leggenda nei primi tempi : più tardi mentre la letteratura puramente ecclesiastica dell' XI e XII secolo che si trora molto sotto l'influenza bizantina l'accetta, quella storica ritiene sempre la vera versione fino al XIII secolo. Di cio Eutichio arcivescovo di Alessandria (c. 975) e Giorgio al-Makin (c. 1250 †1273) sono due chiari esempi. Eutichio nella sua cronaca narra che Costantino abbraccio il cristianesimo nella città chiamata Nicomedia nel duodecimo anno del suo regno, e Giorgio al-Makin copia le sue parole quando dice che "l'imperatore Costantino si fece cristiano in Nicomedia nel duodecimo anno del suo regno »('). Invece nel Sinassario della chiesa copta Alessandrina compilato da Michele vescovo di Atriba e di Mâlig in Egitto (c. 1100) si legge, al 12 di Messori (agosto) che «Costantino è asserito esser stato, nell'undecimo anno del suo impero, battezzato da s. Silvestro papa di Roma ( $\left.{ }^{2}\right)$. Sembra chiaro aver la letteratura storica di Egitto seguito le antiche fonti storiche poichè non vi fu mai tradizione popolare riguardo alla conversione ed il battesimo di Costantino ed era fuori dell' influenza del resto dell'Oriente e specialmente della Siria. Al contrario la letteratura puramente ecclesiastica si confermo moltissimo alla greca ed è probabile che siasi a questo modo introdotta la leggenda di Costantino.

Per colmare la lacuna di cui abbiamo parlato potremmo addurre, oltre il Sinassario copto, anche i menei siri melchiti, ma essi non sono che semplici traduzioni dal greco e percio di poco momento. Leggiamo p. e. nel meneo Melch. abbreviato (contenuto nel cod. Sir. vat. 351, f. 259), al 21 del mese Aiar, la commemorazione di Costantino e di Elena ove occorre questo passo (1): (

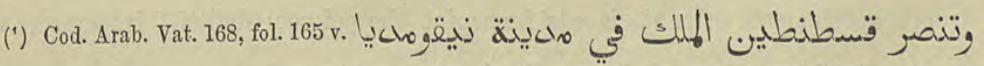

(") Cod. Arab. Vat. LXIII, fol. 166.

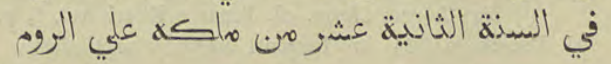




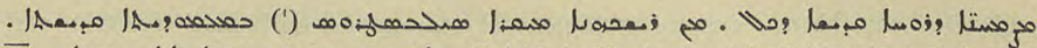

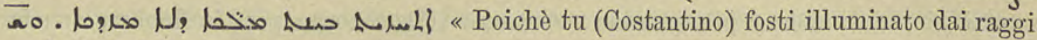
"gloriosi dello spirito santissimo per il venerabile pontefice Silvestro col santo bat« tesimo, sei divenuto fra i re l'invitto ecc.».

Più tardi nel medesimo secolo il famoso Gregorio Barebreo riporta la leggenda, ma in modo da mostrare come eransi confusi nella mente dei Siri tutti gli avvenimenti di quell'epoca. Nella parte I ${ }^{a}$ della sua cronica ( $\left.{ }^{1}\right)$ leggesi: « Dopo Diocleziano « Cesare ed i suoi associati venne Costantino il grande il quale sotto Diocleziano « aveva regnato nella Gallia, e fu padre di Costantino il vittorioso ....... e questo « Costantino il grande fu istruito nella fede da Silvestro veșcovo di Roma, poichè « era lebbroso, e fu convertito, battezzato e guarito ........ Dopo di lui regnò il suo figlio per trentadue anni ........ « quando stava per combattere il tiranno Mas« senzio vide di giorno nel cielo una croce brillante come fuoco ecc. ». In questo racconto Gregorio attribuisce al padrè di Costantino, al quale dà il medesimo nome, le circostanze della leggenda.

Chiuderò dicendo che la leggenda passò in una forma modificata anche agli arabi. Difatti la troviamo riportata nella cronica di Ibn-al-Athir ( 11231 ) in termini però molto vaghi, e non vi si parla di Silvestro ma d'un Veziro come causa della conversione $\left({ }^{2}\right)$. « Si disputa sulla causa della sua conversione (di Costantino) " al cristianesimo. Alcuni dicono che era affetto di lebbra che cercava di sradicare. " Allora un suo veziro il quale era fra coloro che segretamente erano cristiani gli « consiglio di adottare una nuova religione che egli propugnerebhe, e gli magnifico « la bellezza del cristianesimo, e che lo aiuterebbero coloro che ne erano seguaci. « Cio facendo (l'Imperatore) lo seguirono i romani cristiani i quali si unirono al " suo esercito ecc. », e per risultato vince i suoi nemici.Poscia Ibn-al-Athir dà un'altra versione secondo la quale, mentre il suo esercito stava sotto la protezione di sette idoli (secondo le opinioni dei Sabei) fu vinto, ma facendosi cristiano l' Imperatore per suggerimento di un suo veziro, diviene vittorioso. Quest' ultimo racconto deriva chiaramente dalla visione della croce.

Dalle citazioni da noi date appare chiaro che la leggenda non ebbe mai in Oriente nè quella diffusione generale nè quel carattere fisso che troviamo fra greci e latini. Ogni scrittore vi attribuisce particolarità che sono a lui proprie, e non pare che la leggenda abbia mai preso fra loro molta importanza.

Terminato l'esame delle varie letterature è d'uopo riepilogare le conclusioni che si devono da esso trarre sull'origine e lo sviluppo della leggenda. È certo che essa non fu concepita di un solo tratto nella forma in cui la troviamo negli Atti: essa ebbe origine, come abbiamo detto, poco dopo il 400 fra i greci ortodossi, i quali accolsero probabilmente con favore le voci fra loro nate per lo scopo di vendicare la memoria dell' Imperatore dalla taccia d'arianesimo. Bisogna a mio avviso distinguere tre fasi: $1^{\circ}$ La leggenda ebbe verosimilmente. una causa che non urtava troppo apertamente colla storia e s'immaginò che pei suoi delitti l'Imperatore trovò

(') Bar-Hebraei Chron. ed. Bruns e Kirsch vol. I, p. 64 e v. II, p. 61: cfr. la cronaca Araba (ed. Pococke p. 134) ove leggonsi maggiori particolari.

(') Ed. Tornberg. vol. I, p. 235. 
purificazione nella sua conversione e battesimo ('); $2^{\circ}$ Poscia l' impurità morale si sviluppò in un' impurità fisica, la quale però aveva un senso símbolico; la lebbra rappresentando la falsa religione in cui era nato l'Imperatore e che col battesimo fu sradicata $\left(^{2}\right) ; 3^{\circ}$ Finalmente non si considerò più la lebbra che nel senso fisico come vediamo negli Atti. Quest' ultima forma non fu però universalmente adottata: gli scrittori greci, fino all'ottavo secolo, seguirono sempre la forma più antica.

Quanto allo sviluppo della leggenda essa, nei primi tempi, trovò per così dire una diffusione spontanea ed universale, e la vediamo in tutte le letterature orientali ed occidentali, ma più specialmente in scrittori greci ed orientali. Nel sesto secolo comincia a perdere terreno presso i greci e nel settimo non ne vediamo che poche traccie nella loro letteratura.

Mentre, dopo essersi sviluppata da una forma ad un'altra in Oriente e fra i greci, ivi quasi si estinse, il contrario si osserva fra i latini: essi furono più lenti ad adottarla, ma per ragioni chiare, gli serittori ecclesiastici d'Occidente vi diedero sempre più importanza e appoggio fino che nell' VIII secolo non fu più da nessuno contestata. Questo stato di cose nella letterarura latina produsse per mezzo della lettera d'Adriano una reazione fra i greci, i quali alla fine di quel secolo ricominciarono ad occuparsi della leggenda, che da quel tempo prese fra loro profonde radici, ma che essi non adottarono sempre nella forma puramente identica a quella degli Atti. Le letterature orientali la ricordarono fino all' ottavo secolo (Dionisio), ma nei secoli posteriori non ne troviamo traccia, e non fu che più tardi, nel XIII secolo che la leggenda vi trovò di nuovo favore. La grande importanza data alla leggenda dai latini, e l'influenza che essi esercitarono posteriormente sopra i greci, è stata probabilmente cio che ha indotto nell'errore di supporla di origine romana.

\section{Della leggenda nei monumenti e nell'arte.}

Ho creduto bene aggiungere qualche cenno sopra alcuni dei monumenti più importanti ove trovasi illustrata la leggenda, senza cercare però a darne una lista completa, o anche numerosa. Era costume degli autori medioevali e degli scritti apocrifi di addurre il Battistero del Laterano come prova monumentale del battesimo. Che questa tradizione non abbia valore è provato dagli Atti stessi i quali, se vi fosse stata una tale tradizione, non avrebbero mancato di approfittarne. Il « Liber Pontificalis » 0 piuttosto il «Libellus de munificentia Constantini » per primo parla del fonte battesimale ove fu battezzato l'Imperatore, e questa notizia è ripetuta in scrittori posteriori: ma fra i greci Teofane è il primo a parlare del Battistero, ed altri seguono il suo esempio. È notissimo che la ragione perchè il Battistero ha ricevuto il nome di Costantiniano fu la medesima per la quale fu chiamata anche Costantiniana la Basilica Lateranense, cioè perchè fu la prima chiesa da Costantino fondata. Troviamo un esempio del modo con cui tutto cio che apparteneva al Laterano era associato a Costantino nella statua stessa di Marco Aurelio che, per la sua posizione presso la chiesa, fu creduto nel medio evo rappresentare il primo imperatore cristiano. Lasciamo

(1) Zosimo e Sozomeno. - (־) Giacomo di Sarûg. 
quindi da parte il battistero e veniamo ai monumenti che veramente riguardano la leggenda. Nella chiesa di s. Polyeucto a Costantinopoli, fondata sulla fine del V secolo da Giuliana Anicia figlia di Olibrio, imperatore d' Occidente (472), vi era un affresco o un mosaico che rappresentava il battesimo di Costantino. La memoria ce n'è conservata in un epigramma dell'antologia greca ('), e si disputa se esso indichi che il battesimo era quello storico o quello leggendario. Il canonico Bock $\left({ }^{2}\right)$ sostiene la prima teoria: l'ab. Duchesne $\left({ }^{3}\right)$ senza negarne la possibilità è disposto a vedervi il battesimo storico: ecco intanto l'iscrizione,

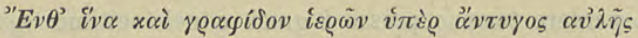

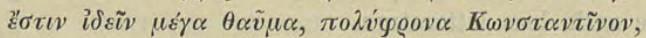

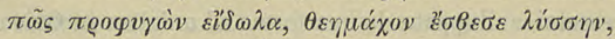

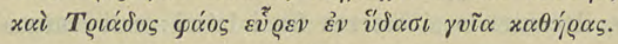

Credo che dalle parole del testo si possano dedurre prove indirette ma quasi certe a favore della leggenda. Poichè supponendo per un momento che rappresen-

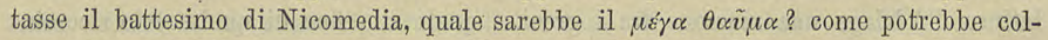
legarsi, quasi fatto contemporaneo, alla conversione ed al ricevimento della conoscenza della SS. Trinita, cose che non ebbero relazione veruna al battesimo storico? perchè, finalmente, direbbesi che Costantino si purificò il corpo, mentre il sagramento del battesimo era preso sempre nella chiesa come purificatore dell'anima? $\mathrm{Al}$ contrario tutti questi fatti nella loro relazione si spiegano con ogni facilità se supponiamo che illustrino la leggenda. Qual grande meraviglia, difatti, nella guarigione del corpo dell'Imperatore dalla lebbra quando si decise finalmente a fuggire gl'idoli, e che fu istruito da s. Silvestro nei misteri della religione e nella dottrina

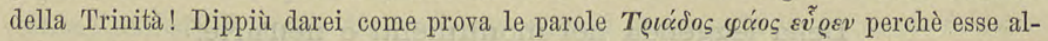
ludono chiaramente ad un incidente del battesimo che trovasi non solamente negli Atti greci latini e siriaci ma anche nell'Omelia di Giacomo di Sarûg, il che ne mostra l'accettazione generale: quando sta per compirsi l'atto del battesimo gli Atti greci (")

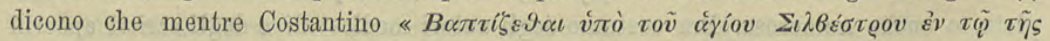

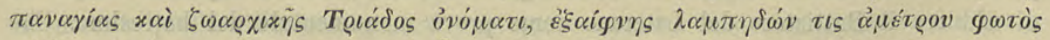

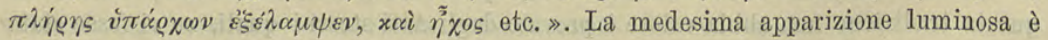
descritta nei versi 647-650 della nostra Omelia. Sembra dunque che tutte le probabilità siano in favore della leggenda, e in questo caso, un tal fatto sarebbe importantissimo poichè mostrerebbe il favore che godeva la leggenda nella Città imperiale ad un tempo quando a Roma si principiava solamente a sostenerla e con qualche riserva, e sarebbe anche nuova e riguardevole prova della sua origine greca.

Di data incerta è la famosa iscrizione che ancora vedesi nell'attuale facciata della basilica Lateranense $\left({ }^{5}\right)$ :

(') Ed. Jacobs, T. I, p. 8. - ( $\left.{ }^{2}\right)$ Christliche Kunstblätler di Freiburg-in-Breisgau, gen. e feb. 1869, p. 145-151; cfr. Greg. Turon, De Gloria Marl. 1. I, c. 103. - (") Élude sur le L. P. p. 172, 173. (') Combefis, op. cit. p. 282. - ( ${ }^{5}$ ) Rohault de Fleury, Le Latran au Moyen-Age. Paris 1877, p. 416; cfr. Rasponi, De Basil. el Palr. Lat. 1656, p. 14. 
AGNOSCANT CVNCTI SACRO BAPTISMATE FVNCTI

QVOD DOMVS HAEC MVNDA NVLLI SIT IN ORBE SECVNDA ete.

$\ldots \ldots \ldots \ldots \ldots \ldots . \ldots \ldots . \ldots . \ldots . \ldots . \ldots$

HANC CONSTANTINVS IN COELVM MENTE SVPINVS

LEPRA MVNDATVS INTVS FORISQVE NOVATVS

FVNDAVIT PRIMVS etc.

A questa era accoppiata un'altra del 430 ;

FLAVIVS CONSTANTIVS FELIX VICTOR MAGISTER etc.,

e questa medesima data fu assegnata alla prima dal Ciampini: il che è cosa impossibile; e lo stesso Rohault de Fleury (che accetta ancora per vera la leggenda!) parve dubitare se non fosse più moderna ( $\left.{ }^{1}\right)$. L'Assemanni inclina a crederla del tempo di Sergio III (904): ed infatti più antica di questo pontefice è difficile che possa essere, poichè versi leonini come quei che in questa iscrizione occorrono, non sono adottati fino al mille incirca.

Di data certa è una iscrizione di Sergio III (904), il quale, com' è notissimo, rifece la basilica dopo la sua rovina cagionata dal gran terremoto dell' 895 . L'iscrizione era a mosaico e stava nell'abside ove esistè fino che essa fu rifatta sotto Nicold IV $\left({ }^{2}\right)$.

\section{AVGVSTVS CAESAR TOTVM CVM DVCERET ORBEM \\ CONDIDIT HANC AVLAM SILVESTRI CHRISMATE SACRAM \\ IAMQVE SALVTIFERA LEPRA MVNDATVS AB VNDA \\ ECCLESIAE HIC SEDEM CONSTRVXIT PRIMVS IN ORBEM \\ SALVATORI DEO etc.}

Le opere d'arte a cui si riferiscono queste iscrizioni sono perdute. Venendo ora alla descrizione di quelle che ci sono rimaste, osserveremo come nei monumenti occidentali gli Atti di s. Silvestro sono, come è naturale, in ogni particolare seguiti.

L'opera più antica che conosciamo ove sono raffigurati alcuni tratti della leggenda è un architrave di marmo che anticamente ornava la porta della chiesa di s. Silvestro a Pisa, prima dei ristauri eseguiti dal granduca Leopoldo: esso si trova ora nel Campo Santo. Il Lasinio $\left({ }^{3}\right)$ lo attribuisce al X secolo, e lo stile concorda perfettamente con quell'epoca: le figure sono rozze e mancano assolutamente di proporzione. Il marmo si divide in due bande di rilievi ripartiti, senza separazione veruna, in dieci compartimenti, quattro sulla linea superiore e sei sull'inferiore, i quali rappresentano: 1) Le madri coi loro fanciulli condotti all'Imperatore; 2) L'apparizione in sogno a Costantino di s. Pietro e s. Paolo; 3) L'Imperatore comanda ai suoi servi di richiamare Silvestro; 4) Silvestro condotto all'Imperatore; 5) Si mostrano le immagini degli Apostoli; 6) Battesimo di Costantino; 7 e 8) Disputa degli Ebrei con Silvestro avanti l'Imperatore; 9) L'Ebreo susurrando nell'orecchio del toro; 10) Silvestro ed il drago.

Del medesimo secolo è un manoscritto dell'archivio Capit. d'Ivrea, fatto scrivere

(') Ibid. p. 30. - (') Ibid. p. 44\% - (") Raccolla di sarcofagi, urne e allri monumenli di scullura del Campo Sanlo di Pisa, intagliati da Paolo Lasinio figlio. Pisa 1844, tav. C. 
dal vescovo Warmundo ( $\$$ 1004) ('). Esso contiene una serie di disegni accompagnati d'iscrizioni, fra i quali si vede: 1) S. Silvestro che guarisce l'imperatore Costantino dalla lebbra e leggesi, Lepra cadit mentis redeunt precamina membris; 2) Nella seguente composizione si vede l'Imperatore al lavacro, Mentibus ardorem pedibus das Christe nitorem.

A quest'epoca i monumenti si moltiplicano e non potremo darli che in parte. Di maggiore antichità erano forse alcuni affreschi dell' antica facciata di s. Pietro. Il Grimaldi, descrivendone i musaici eseguiti sotto Gregorio IX (1227 - 1241), viene a parlare di antichi affreschi che vi sono sottoposti ( $\left.{ }^{2}\right)$, dicendo, "Inter ipsa stem«mata et arcus columnarum porticus cernuntur historiae antiquissimae B. Petri, « vel ab eodem Gregorio vel ab alio antiquiore pontifice factae, sunt autem disputatio « cum Simone Mago ...., visio Constantini de Sanctis Apostolis, ostensio imaginum « ipsorum Constantino, prout clarius in libro picturarum in dicto Archivio apparet ».

La facciata di s. Giovanni Laterano come esisteva ancora nel XVI secolo, era ornata d'un portico, sopra il cui architrave correva una fascia di musaico ed i cui compartimenti erano separati da medaglioni. Alcuni di questi rimanevano nel tempo del Ciampini il quale ce ne ha dato $\mathrm{i}$ disegni $\left(^{3}\right)$ : sono ritratti con molto maggiore accuratezza in disegni colorati della grandezza dell'originale che trovansi in un cod. Barberiniano e che sono di quasi un mezzo secolo anteriori al Ciampini. Si vede rappresentata in un compartimento il battesimo di Costantino, in un altro l'atto di donazione dell' Imperatore a Silvestro, e finalmente s. Silvestro ed il drago.

Sotto il musaico, sul pilastro angolare, leggevasi il nome dell'artista nicolavs ANGeli fECIT ноç opvs. Quest'opera Rohault de Fleury ( ${ }^{4}$ ) erroneamente assegna a Nicold IV (1288-92), e crede l'artista un Toscano; al contrario abbiamo mostrato ch'egli è Romano e fiorì nella seconda metà del XII secolo $\left({ }^{5}\right)$. Il nome di Niccolò figlio di Angelo è notissimo nella storia medioevale dei marmorari romani; fu egli che scolpì il cereo pasquale di s. Paolo, fece l'altare della Cattedrale di Sutri nel 1170, architetto la confessione di s. Bartolomeo all'Isola nell'1180. Ora dobbiamo aggiungere il portico coi mosaici di s. Giovanni Laterano, lavoro eseguito probabilmente sotto Adriano IV (1154-59) e forse il primo lavoro che di lui conosciamo. Si mostra chiaramente essere la produzione di una scuola schiettamente nazionale e non rivela traccia veruna d'influenza bizantina.

Ma l'opera più importante per l'illustrazione degli Atti sono gli affreschi dei quali sono coperte le mura della cappella di s. Silvestro ai ss. Quattro Coronati. Una iscrizione del 1248 ci indica la loro epoca. Le composizioni sono nel numero di dieci, e rappresentano: 1) Costantino nel suo carro incontrando le madri coi loro figli; 2) Gli appariscono in sogno i ss. Pietro e Paolo; 3) I suoi messaggeri vanno a ricercare s. Silvestro; 4) Essi arrivano al Soratte e trovano Silvestro; 5) Il Pontefice

(') C. Gazzera, Delle iscrizioni cristiane antiche del Piemontc. Torino 1849, p. 112. - ( ${ }^{\mathrm{a}}$ ) Eug. Müntz, Recherches sur l'œuivre archéol. de Jacques Grimaldi, nella Bib. des Écoles Franc. d'Athènes et de Rome, fasc. I, p. 257. - (") De sacris Aedif. p. 10-13, tav. I, II. - (") Le Lalran au Moyen Age, Paris 1877, p. 180; cfr. p. 334, 335. - $\left(^{5}\right)$ Nel gennaio passato lessi alla Socielà dei cultori dell Archeologia cristiana una comunicazione sopra questi musaici dimostrando l'identità dell'artista il quale li lavorò col noto marmorario romano. 
mostra a Costantino le immagini degli Apostoli; 6) Battesimo dell'Imperatore; 7) La sua coronazione fatta da 's. Silvestro; 8) 9) 10) Rappresentano la disputa di s. Silvestro e degli Ebrei, la morte e risuscitazione del toro; ed il miracolo del drago.

Le arti d'oltr'alpe non mancarono di raffigurare una leggenda cosi popolare. Difatti in una delle fenestre della cattedrale di Chartres, i cui vetri dipinti rimontano al XIII secolo, vediamo la storia di s. Silvestro e Timoteo, ecc., con ogni particolare rappresentata in trentuna composizioni. Il coro del duomo di Colonia consacrato nel 1322 è ornato di affreschi importantissimi anteriori a quell'anno: essi si dividono in quattro serie; l'ultima di queste contiene in sette composizioni la leggenda di s. Silvestro. Il Förster (') che le ha descritte non sembra però conoscere gl'incidenti degli Atti e perciò alcuni soggetti rimangono oscuri. Si vedeva: 1) L'ingresso di s. Silvestro al monastero; 2) «Silvestro conducendo Simmaco da un monaco »; 3) L' insegnamento ed il martirio di Timoteo; 4) L'arresto di Silvestro; 5) * Il papa Melchiade $\left({ }^{2}\right)$ che libera Silvestro dalla carcere »;6) Silvestro eletto papa dopo la morte di Melchiade $\left({ }^{2}\right)$; 7) L'imperatore Costantino $\left({ }^{3}\right)$ che comanda di sgozzare i fanciulli.

Non continueremo più oltre l'enumerazione dei monumenti. Quantunque non abbiamo raccolto che una piccola parte delle notizie che potrebbero trovarsi, cio che abbiamo detto mostra chiaramente quale sia l'importanza della leggenda per la storia medioevale; mostra che essa aveva penetrato tutte le classi: che i sacerdoti l'insegnavano, gli scrittori la narravano, e gli artisti la rappresentavano agli occhi di tutti.

Mi rimane a ringraziare caldamente il prof. I. Guidi pei consigli da lui avuti in questa pubblicazione che egli ebbe la bontà di presentare all'Accademia dei Lincei.

\section{B I B L I O G R A F I A}

Cusa Nicolaus de († 1464) De Concordantia Catholica. Judicium de donatione Constantini. Basil. 1565. Zenus Jacobus, Episc. Patav. († $147 \%$ o 1481) De vitis pontificum ad Paulum papam.

Valla Laurentius (c. 1444) De falsa donatione Constantini.

Jacobatii Card. Dominic. († 1528) De Concilio Tractatus, Romae 1538: Lib. X, art. 8, p. 780-783. De Donatione Constantini.

Polus Card. Reginaldus (c. 1550) Liber de Concilio, de Baptismo Magni Conslantini Imperaloris ecc. in Conc. Labbei, vol. XIV, p. 1660.

Baronius Card. Caesar (1538-1607) Annales Ecclesiastici, ad an. 323-324.

Scultetus Abraham, Confutatio Caes. Baronii de Baptismo Constantini. 1607.

Morin Jean, Histoire de la délivrance de l'Eglise Chrestienne par l'Empereur Constantin ecc., Paris 1630. Schelstrate D. Emanuel a Antiq. illusl. circa Concil. Gen. ecc., et praetipua tot. Hist. Eccles. cog. Antw. 1678. P. II, diss. III, cap. VI. De Baptismo Constantini num Romae a S. Silvestro, num Nicomediae ab Eusebio collatus fuit, an potius et Romae et Nicomediae.

Finckius D. Casp. De Dispul. de Baptismo, T. V, p. 313, disp. XIII.

Kunadus D. And. Canstantinus Magnus Evangel. Diss. I, p. 13. Witeb. 1664.

(') E. Förster, Monuments d'Arch. de Sculp. et de Peint. de l'Allemagne. Paris 1859: Painture, T. II, p. 49 e 50. - ( $\left(^{3}\right)$ L'autore dice, Melchizedech ma ciò è senza dubbio uno sbaglio. - (") Il Förster lo crede «l'empereur Constantin donnant l'ordre de baptiser plusieurs enfants »! 
Noris Card. Henricus, Hisloria Donatist. 1681, op. T. IV, p. 650.

Sagittarius Christ. Dissert. Hist. de Constantino Magno. Jen. 1650.

Tentzel (W. E.) Exercil. selectae ecc. Lipsiae et Francof. 1691, p. 299-599. Exercitatio oclava sive Examen Fabulae Rom. de Duplici Bap. Constantini Magni adversus defensionem Cl. v. D. Eman. a Schelstrate.

Pagi Anton, Crit. historico-chron. in Ann. Baronii, ad an. 323-324. Ginevra 1705.

Bini Severini, Nolae ad notiliam Libri Pontificalis (Vita di Silvestro) Migne, Patr. Lat. T. VIII, pp. 795-800.

Fabricius I. A. Salularis Lux Evangelii, Hamb. 1731; e le opere ivi menzionate, p. 260-265.

Fuhrmann Mathia, Historia sacra de Baptismo Constanlini Max. Aug. Colloquiis familiaribus digesta, complectens verum ac unicum Magni hujus Imperaloris Baptismum Romanum : 2 tom. Romae 1742 et Viennae 1746.

Mamachius Thom. Maria, Orig. et antiq. Christ. T. II, p. 232 sgg. Roma 1750-54.

Döllinger Joh. Jos. Ign. von Die Papst-Fabeln des Mitlelallers: München 1863 (p. 52 sgg.).

Maury, Essai sur les légendes pieuses au Moyen-Age. Paris p. 65 ecc.

Bock canon. Chrislliche Kunstblätler 1869 Jan. Feb. Freiburg-in-Breisgan. Die Kirche des hl. Polyeukt in Constantinopel.

Duchesne Abbé L. Étude sur le Liber Ponlificalis. Bib. des Écoles Franç. d'Athènes et de Rome fasc. I. Paris 1877 , p. $165-173$ ecc.

Guidi Ignazio, La descrizione di Roma nei Geograf Arabi. Roma 187 a cura della Soc. Romana di Storia Patria, p. 43-46 (').

\section{Descrizione dei Manoscritti.}

L'Omelia di Giacomo di Sarûg sul battesimo di Costantino è contenuta in due soli codici $\left({ }^{2}\right)$, il Vat. Siriaco $117\left(^{3}\right)$, cioè, ed il Brit. Museum 803 (add. 14588) ( $\left.{ }^{4}\right)$; oltre questi due testi ne troviamo un frammento nel Cod. 153 della Bibl. Bodleiana a Oxford $\left({ }^{5}\right)$.

Il testo Vaticano è intiero ed è quello che seguiamo nella nostra pubblicazione: esso occupa i fogli $542, a 2-546, b 2$, del codice il quale contiene una preziosa collezione di omelie. Questo codice non è stato mai sufficientemente descritto, nè la sua età precisamente determinata: cercheremo percio di supplire a cio che ne è stato detto da Assemanni e coloro i quali non hanno fatto generalmente che copiare le sue parole, come Mösinger $\left({ }^{\circ}\right)$, Abbeloos $\left({ }^{7}\right)$ ed altri. Il manoseritto consiste di 559 fogli a tre colonne e non di 555 come asserisce l'Assemanni, nè

(') Di tutte queste opere, le antiche non mi hanno giovato che coll'indicazione di qualche fonte; fra le moderne, poi, nelle quali la questione viene trattata criticąmente, non havvene veruna che abbia sostenuto l'origine greca della leggenda come mi sono sforzato di fare. - (') Ho trovato che il cod. Vat. 116, nel quale, di 20 omelie di Giacomo di Sarûg che prima leggevansi, non rimangono ora che le quattro ultime, conteneva la nostra Omelia: questo apprendiamo dall'indice, al fol. 42, ove leggiamo: les omelia sopra Costantino imperatore lebbroso ». Il codice è dell' anno 857 (cfr. Assemani, Catal. T. III, p. 86-87) ed è forse uno degli antichi codici che servirono alla compilazione del cod. 117. (") J. S. Assemanni, Calal. T. III, p. 87; cfr. Bibl. Orient. T. I, p. 328 ecc. - (') W. Wright, Catalogue of the Syriac MSS. of the Brit. Mus. p. 80\%. - ( () R. Payne-Smith, Cod. Manusc. Bib. Bodleian. Pars sexta, cod. Syr. ecc. p. 49\% - (') Monumenta Syriaca, OEmp. 1869, T. I. - (') Op. cit. p. XV-XVII. 
di 558 come indica Ia paginazione a lui posteriore: poichè nella prima non si principio la paginazione ai primi fogli, e nella seconda, per errore, fu omessa la cifra 241. Ogni colonna contiene da 42 a 49 righe. Il numero dei quinterni è di 52 di dieci fogli ciascuno, cio che darebbe 520 fogli solamente al manoscritto primitivo. Difatti un esame attento ci mostra essere stati inseriti nella legatura parecchi fogli provenienti da altri mss. Cì è specialmente evidente pei fogli 110-114 contenenti la vita di s. Giacomo scritta da un suo discepolo Giorgio ('), ove troviamo una scrittura delicata ed irregolare, ed una carta piu sottile e ruvida, 5 cent. meno larga che quella del rimanente del volume. Gli altri fogli inseriti sono più difficili a distinguersi, ma pare che si trovino nei quinterni $\wp^{\circ}, \downarrow, \infty,+, 0$, ๑ $\mathrm{e}, \hookleftarrow, \lambda$ mentre, al contrario, ne manca uno nei quinterni la e Finalmente la data del codice non era stata trovata; l'Assemanni lo crede scritto verso il 1100 , altri lo dicono del XII secolo. Essa però può essere precisata dal codice stesso. Sul verso dell'ultimo foglio (del quale daremo in appresso l'iscrizione centrale), troviamo nell'angolo inferiore di destra una mutilissima iserizione in un circoletto del diametro di 6 cent. Essa consisteva di dieci righe: la metà esterna

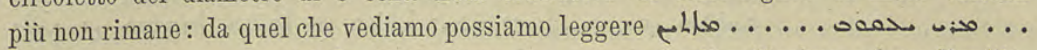

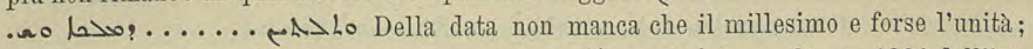

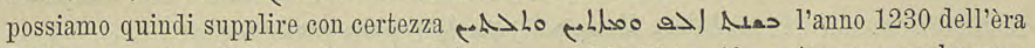
dei Greci, cioè A. D.919. Supponendo che manchi l'ultima cifra potremmo prolungare l'epoca possibile fino al 927, anno in cui fu fatto abbate il famoso Mosè di Nisibi.

Una conferma indiretta si ricava da un ms. del Brit Mus. oriundo dal medesimo monastero di Mar Beswai, il quale ci mostra che un Jûhannan bar Macarios era abbate nel 894 A. D.; ora un Jûhannan è dato precisamente come abbate nell'annotazione del nostro codice: pù̀ essere il medesimo, poichè non è inverosimile che fosse ancora in vita nel 919. L' iscrizione centrale occupa quasi intieramente quest'ultimo foglio: essa contiene l'invocazione dello scrittore; racconta la sua entrata nel monastero: il desiderio espresso dall'abbate perchè scrivesse il codice: e l'uso che fa l'amanuense per il suo lavoro di antichi manoscritti già esistenti nel monastero; cio che era gia stato commemorato due volte nelle annotazioni ai fol. 117 e 501 . Termina menzionando l'abbate, l'economo ed i monaci allora viventi nel monastero.

Assemanni $\left({ }^{2}\right)$ pretende dare tutto cio che è leggibile di quest'annotazione, ma ne fa un compendio ove non mette che una terza parte delle parole esistenti, e fra altre, tralascia i nomi di sei dei 23 monaci compreso anche l'abbate, cambiandone inoltre l'ordine e prendendo come nome proprio le parole la la is quali sono precedute da un punto e principiano un'invocazione a Cristo. Il Mosè qui annoverato è probabilmente Mosè di Nisibi, il quale entrò nel monastero nel 907.

Daremo l'annotazione per intero, per quanto lo permette lo stato attuale di questo foglio, ove molte parole mancano affatto ed altre sono assai incerte: le parole in parentesi sono quelle che si possono con qualche certezza supplire, sia dal senso, sia dal compendio dell'Assemanni, al tempo del quale esistevano molte più parole.

(') Pubblicata dall'Abbeloos, op. cit, p. 24 a 84. - (') Catal. T. III, cod. 11\%, p. 87 e segg. 


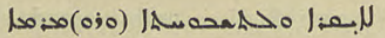

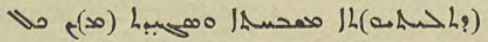

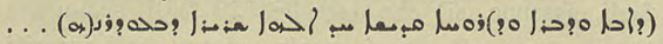

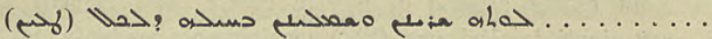

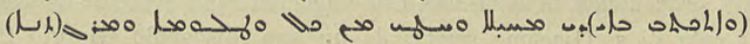

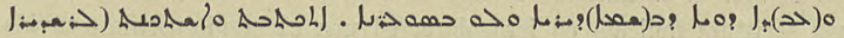

?

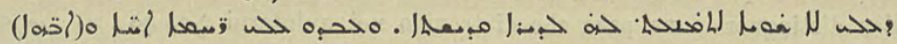

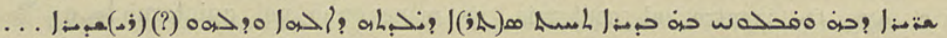

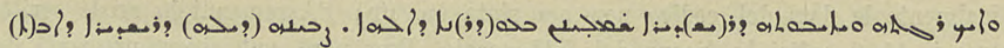
.... . . (A) (a)

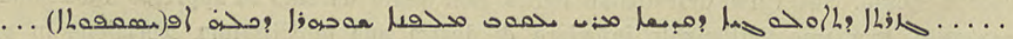

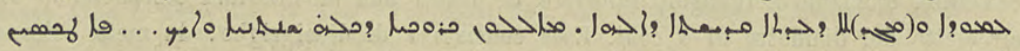

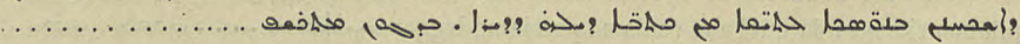

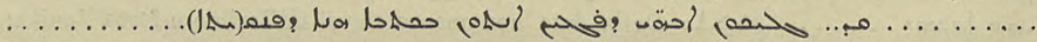

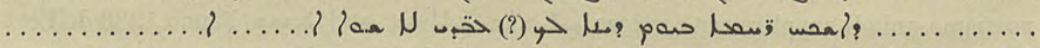
wooj

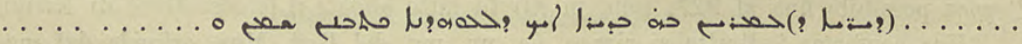

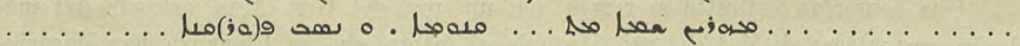

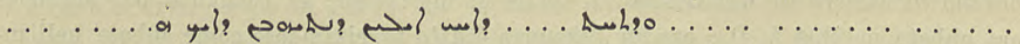

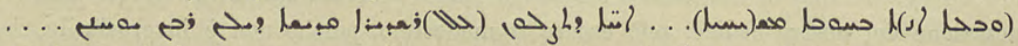

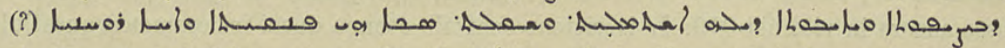

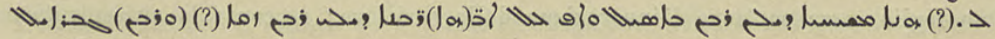

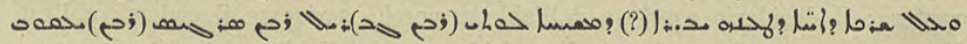

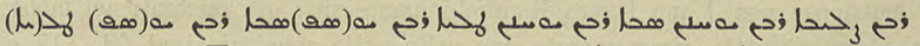

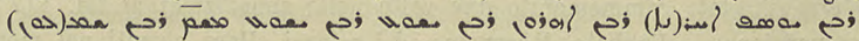

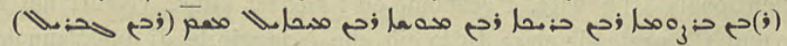

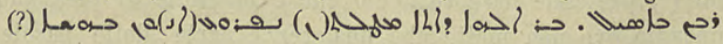

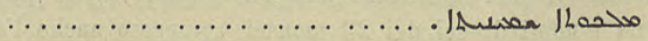

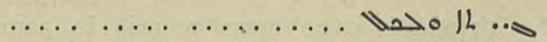

$$
\begin{aligned}
& \text { o }
\end{aligned}
$$

Il Codice 803 del Brit. Museum è della medesima età del Vatic. anzi il chiar. prof. W. Wright crede che possa essere, forse, anche del IX secolo: è scritto in carattere estrangelâ, mentre il Vat. lo è in Sertâ. Questo codice è alquanto monco nella parte contenente la nostra Omelia, ove mancano un foglio in principio ed altri due $o$ tre nel corso del testo. Percio, quantunque la lezione sia probabilmente migliore, non ne abbiamo potuto fare la base del testo. Esso fu per noi collazionato dal prof. W. Wright, il quale, con somma gentilezza, ha voluto adoperarsi 
per farne un lavoro accuratissimo come, è inutile dirlo, sono tutti i suoi lavori: glie ne presentiamo ora i nostri più caldi ringraziamenti. Ne noteremo le varianti colla lettera $\mathrm{M}$; eccetto nei casi ove la differenza è semplicemente ortografica ( $\left.{ }^{1}\right)$. Il gran numero dei luoghi ove i due codici ci danno una narrazione radicalmente diversa mostra quanto incerto sia il testo, ed è anche una indicazione della popolarità e propagazione del testo.

Il Codice Sir. 153 della Bib. Bodleiana di Oxford contiene, ai fol. 13-16 una composizione intitolata:

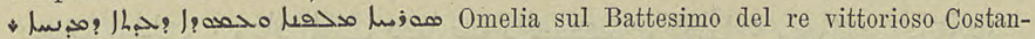
tino; di Mar Ephraem il Siro, dottore e colonna della chiesa orientale.

Questo fu pubblicato dall'Overbeck ( $\left.{ }^{2}\right)$ sotto il nome di s. Ephraem, ma il prof. Bickell nel suo « Conspectus rei Syrorum litterariae » aveva già notato che non dovevasi a lui attribuire non essendo scritto nel metro da lui adoperato, ma nel metro dodecasillabo quello, cioè, di Giacomo di Sarug. Ora troviamo che questa composizione non è altro che un frammento della nostra Omelia. L'errore del trascrittore nell'assegnarla a s. Ephraem può essere stato cagionato dal fatto che si conosceva aver egli scritto sopra Costantino, come viene accennato nella vita anonima del Santo pubblicata dall'Assemanni e riprodotta nella Crestomazia dell' Uhlemann, la quale se non è contemporanea, poichè vi si parla già degli Stiliti, è almeno del V secolo. Il testo del Codice Bod. è quasi identico in generale con quello del Brit. Mus., e quando ne differisce segue il Vat.: in qualche passo, pero ha un carattere speciale. Esso si estende dai versi 494 a 686 del Cod. Vat. e le varianti sono indicate colla lettera $\mathrm{B}$.

\section{Versione Araba.}

Nel Codice Sir. Vat. 199, scritto in Karsûnì, trovasi una versione Araba dell'Omelia: essa è però moderna, e forse non molto più antica del codice stesso, cioè del 1545. In quanto allo stile, il desiderio del traduttore di aggiungere abbellimenti e fioriture ha reso questa composizione molto più diffusa e noiosa dell'originale. Non ne daremo percio che un breve squarcio, che sceglieremo come saggio di stile: esso corrisponde ai versi 85 a 112 del testo siriaco.

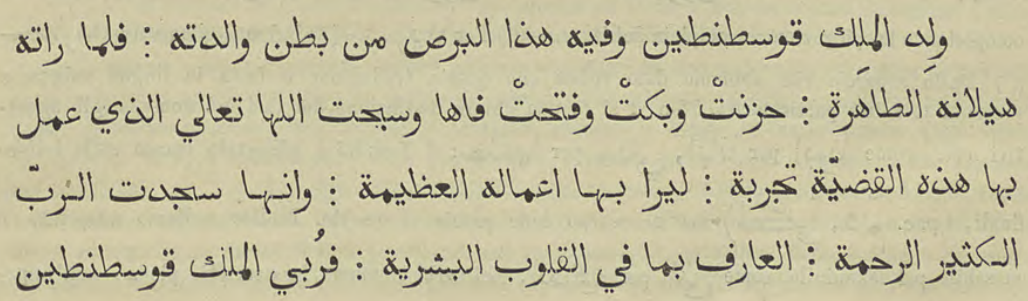

(') e.g. Wo = Mo , S. Ephraemi Syri, Rabulae ecc. Opera selecla, Ox. 1865, p. 355-61. 


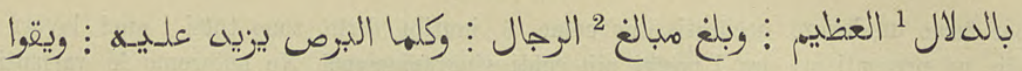

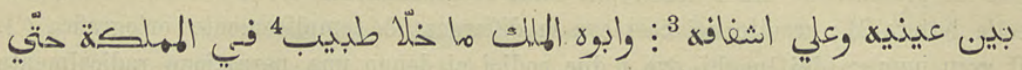
جابه ${ }^{5}$

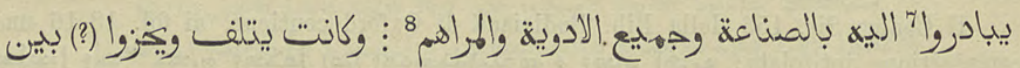

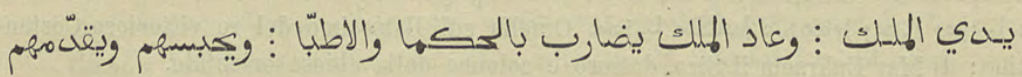
لضرب العنق ويعاقبهم ؛ ويقوّي عليهم الشهايه ؛ وعاد بعضهم يربيه المرت

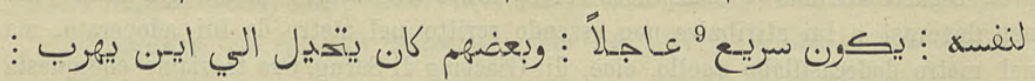

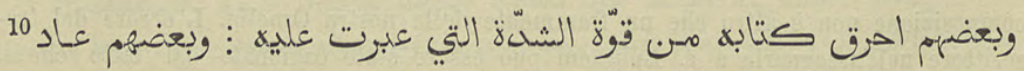

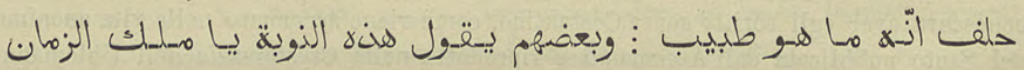

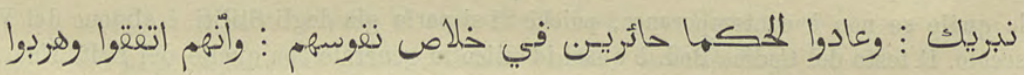

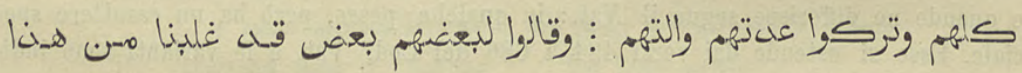

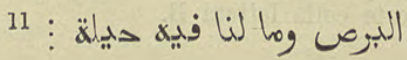

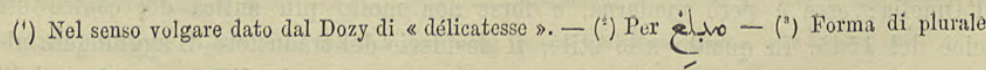

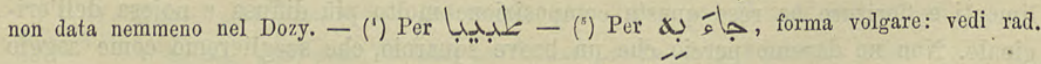
جيب in Dozy. - (') Per Penso di unguento completando la spiegazione incompleta del Dozy. - (') Per Le (1') Dallo squarcio che abbiamo dato vedesi che questa traduzione è fatta in lingua volgare e presenta le solite particolarità. Non vi si fa una chiara distinzione dei casi nel nome, negli agget-

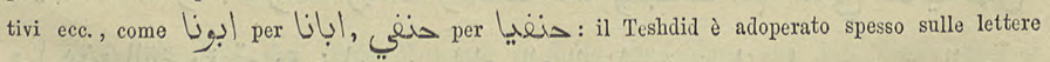
finali, (p.e. دلّ) ma nel corpo delle parole si omette. Inoltre vediamo adoperato il maschile pel femminile, come التي per l. Nei verbi troviamo la «scriptio plena nell’imperativo $\left(ل_{-1-0}=J_{-\infty}\right)$ e nei vẹrbi difettivi $($ ردت = رديـت) i l'elisione della Hamza

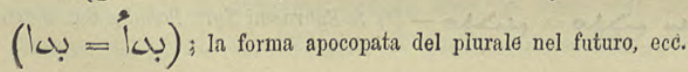




\section{Del medesimo Màr Giacomo di Sarùg. Omelia sopra l'imper. Costantino e la guarigione della sua lebbra (').}

0 Gesù, tu che sei grande luce a chi ha in te lo sguardo, illustra ed illumina me, affinchè io chiaramente di te narri. Tu, o Signore, sei la Via cui non giungono le insidie. In te camminerò e procederò in una narrazione meravigliosa. La storia dell' imperatore Costantino si agita nella mia mente e mi forza, mentre ne stupisco, a farne la narrazione. Ricevi adunque il dono delle-mie deboli parole, poichè (ogni discorso) degli eloquenti senza te è vano. Permettimi, o Signore, di dipingere un'immagine piena di bellezza, affinchè faccia al tuo gregge una narrazione del tutto maravigliosa. Tu sei la Porta, o Signore, permettimi di entrare presso il tuo figlio, e, vicino ad essa, di recitare un discorso intorno al tuo divino operare. Alla tua via non giungono le insidie; per essa camminerò : per te sarò liberato dalle tenebre e (condotto) in una grande luce. Abbi cura, o Signore, del tno gregge che da te fu redento, e per il quale venisti volontariamente in terra. Ecco che il Maligno minaccia tutti i figli di Adamo di precipitarli, se lo potesse, in mezzo all'inferno. Per misericordia creasti il nostro padre Adamo allorchè lo creasti, ed errando egli, lo convertisti, o Signore, col sangue del tuo lato. In ogni bella guisa la ricercasti, quella immagine che le tue mani avevano formata, affinchè non perisse. Vedendo come il Maligno faceva guerra contro Adamo; mandasti il tuo figliuolo affinchè gli venisse in aiuto. Da quel primo delitto imparammo, o Signore, quanto sia abbon- 25 dante la misericordia del tuo amore divino. In varî modi lo incitasti per possederlo, e che non avesse altro Dio che te solo. Vedeva Adamo ch' era giunto alla disperazione e pose l'animo alla penitenza, per mezzo di sacrifici. Ai frutti del suo ventre permise Adamo di sacrificare, pensando che se si convertisse, non sarebbe accettato $\left({ }^{2}\right)$. Fugga (si allontani) ora il grand'Adamo colla sua generazione poichè la penitenza fu accettata. Ora parleremo con amore della generazione Noachitica la quale, essendosi mostrata empia, percotesti col flagello delle acque; (così) fin da tempo antichissimo si moltiplicarono i misteri $\left({ }^{3}\right)$. Poscia, quando si dovè costruire

(') Ci atteniamo in questa traduzione strettamente al testo, e non cerchiamo di togliere i pleonasmi e le ripetizioni, nè dare uno stile più conforme all'indole della lingua italiana.

( $\left.{ }^{4}\right)$ Questo passo riguardante Adamo sembra accennare ad una leggenda, che deve aver avuto corso nei primi secoli, intorno alla caduta dei nostri primi parenti, ed agli avvenimenti posteriori a questo fatto: senza cercare più lontano la troviamo esposta a lungo in varie omelie dello stesso Giacomo di Sarûg, e specialmente nelle quattro ch'egli scrisse sopra Caino ed Abele e che trovansi nel medesimo cod. Vat. 117 (fol. 405 a 411). Ivi leggiamo come, secondo la leggenda, Adamo, riflettendo alle conseguenze della sua colpa, vuol evitare che i suoi figli non pecchino in modo simile nè si attirino la punizione divina: perciò ad essi racconta ogni particolare della caduta e gli esorta ad adorare fedelmente Iddio e renderlo propizio con sacrifizii, cosa che egli stesso colle sue colpevoli mani non oserebbe tentare, certo di non poter ricevere il perdono da Dio, ecc.

(") Con ciò sembra alludere a quel che ha raccontato di Adamo e di Noè come tipi del mistero della redenzione. Si esaminino le varie omelie scritte da Giacomo di Sarûg stesso sopra ognuno dei personaggi qui annorerati. 
l'arca, Noè solo fu trovato giusto nel mondo e nessun'altro, e, poichè era accetto alla tua divinità, salvasti la terra. Retrocedi, o generazione di Noè, dietro i secoli, poichè altre generazioni si affollano dopo to per venire. Vieni, o Abramo, e con amore reca con te la tua generazione, tu da cui a cento anni nacque un frutto, e, poichè molto ti tentò (Iddio), molto amasti la tentazione (?). Passa oltre, generazione di Abramo, e va via dal mio cospetto. Bramo ardentemente narrare con amore la storia 50 della generazione di Asa re d'Israele. Questo giusto non commise iniquità, ma volle far penitenza per un' empietà (non sua) : poichè la sua madre peccò, dalla sua casa stessa scaturiva iniquità. Egli colla penitenza scongiurò il suo Signore di venire a lui. Esci, o Asa, e non indugiare a partire, poichè altre generazioni si affollano e vengono l'una dopo l'altra. Vieni da Gerusalemme, o gran re Ezechia, e conduci con te la penitenza affinchè noi la contempliamo; tu che con una sola preghiera distruggesti colla spada migliaia di uomini. Passate generazioni dei re primi, trascorse da antichissimo, perchè il secolo dell' imperatore Costantino richiede la sua parte. La storia di questo si agita potentemente nella mia mente e mi affretto a narrarla lucidamente. Accorrete, uditori, e prestate un orecchio pieno d'amore: tu anche, o oratore, se parli parla con amore. Fin ad ora ho parlato con voi di molte storie, ma d' ora in poi una sola sarà la storia che tratteremo, parlerò molto sul proposito della penitenza per annunziarla chiaramente agli uditori (').

Dirizziamo la mente alla storia dell' imperatore Costantino, e vedremo come fu da quando uscì dal ventre materno. Il suo Signore lo formò, come fanciullo, nel ventre della sua madre, e come uomo formo in esso l'immagine umana. E, poichè conosceva che il suo padre era gentile $\left({ }^{9}\right)$, pose la lebbra nelle sue membra affinchè 75 non diventasse pagano $\left({ }^{3}\right)$ : collocò cospicuamente la stella della lebbra sulla fronte, ed anche sulle labbra la sparse, e ne rese immonda la favella. Fece divenire uggioso il suo aspetto, fetida la bocca; chi non avrebbe paura? e ciò affinchè, spinto da ambedue queste cose facesse penitenza. Era colpito l'Imperatore da due flagelli senza saperlo, affinchè, conoscendoli poi, si volgesse a penitenza. Era ferito come da una freccia sulla fronte e le labbra, la quale con grande tremore faceva fuggire i suoi amici. Difatti fin dal ventre materno il puzzo della lebbra da lui esalava; uno spettacolo immondo appariva sulla fronte e le labbra e per il suo fetore metteva in fuga chiunque si avvicinava. Nacque dunque Costantino come abbiamo

(') Queste parole mostrano che il nostro scrittore si era già da molto tempo dato alla predicazione, e perciò ci forza di posporre questa Omelia di molto all'anno 472 quando egli cioè recitò la sua prima Omelia sul Carro di Erechiele. Quivi egli annunzia altresì il proposito, che in seguito fedelmente adempì, di consacrare cioè la sua parola ad illustrare sotto ogni punto di vista il soggetto della penitenza.

( $\left.{ }^{2}\right)$ Le varie versioni della leggenda differiscono riguardo ai sentimenti dei parenti di Costantino verso la religione cristiana prima della conversione di lui. Gli Atti fanno credere che Elena fosse a quel tempo favorevole alla religione giudaica (p. 16); secondo Dionigi, ambidue erano cristiani (p. 20). $\mathrm{Ma}$ questo punto fu trattato con maggiori particolari in iscritti che non hanno diretta relazione colla nostra leggenda, come nella leggenda di Elena (vedi Coen op. cit.), ed anche nei documenti riguardanti la leggenda che appartengono al medio evo tardo.

$\left({ }^{3}\right)$ Qnesta opinione che ciò̀ Costantino fu lebbroso fin dalla nascita sembra adottata da alcuni serittori greci posteriori (v. pag. 17). 
narrato, ed uscì dal ventre stesso la lebbra odiata. Considerandolo, sua madre, aprì la bocca per lodare quell'Ottimo il quale gli aveva dato il flagello della lebbra affinchè fosse per esso risanato $\left({ }^{1}\right)$. «Ti adoro, o Figlio di Dio misericordiosissimo poichè « tu conosei il cuore degli uomini ». Cresceva Costantino nel palazzo, e la statura del fanciullo giunse ad essere quella d'un uomo fatto; restandogli sempre la lebbra sulla fronte e le labbra, ed essendo tormentato dal fetore della lebbra maligna. Il padre non lasciò nei suoi dominî un solo medico che nol chiamasse presso sè, ma la lebbra immonda non era vinta a guarigione. Vennero i medici ma l'ulcero maligno (svergognava) resisteva alle loro erbe: (l'Imperatore) li puniva di flagelli, tormenti e battiture, e gli fece morire crudelmente a fil di spada. Alcuni di loro, sen- 100 tendo il comando, chiedevano che la morte loro rapisse prestamente l'anima: altri, poi, meditavano dove fuggire per salvarsi dall'ira che loro sovrastava; chi per dolore bruciava i suoi libri: chi giurava: «io non sono medico»: chi cercava di fuggire e dar luogo ad altri : chi cercava d'entrare (presso Costantino) per guarirlo se lo potesse. Allora i medici parlarono in questo modo $\left({ }^{9}\right)$, dopo abbandonate le loro varie erbe (come inutili): «Fuggiamo, poichè non è possibile di sanare la sua lebbra immonda, nè siamo in grado di dare al fanciullo la guarigione del corpo $\left(^{3}\right)$. Mentre l'Imperatore cercava come potesse guarire la lebbra del suo figlio, (l'Imperatrice) fedele, aflitta, implorava che non guarisse; poichè se fosse risanato mentre era ancor pagano accrescerebbe il male ed il giovane seguirebbe la via del suo padre se fosse guarito. Una preghiera usciva dalla sua bocea e sulle sue labbra era un dolce incenso, supplicando che potesse morire il padre di Costantino. «Deh! che « non cresca il fanciullo con lui, o Signore, nè impari la sua empietà ». Ella non pregava per la morte del fanciullo perchè conosceva l'arcano ch' egli avrebbe poi esaltato la santa Chiesa, e avrebbe represso ogni eresia: percio non pregava per la morte del suo figlio, ma per quella del suo padre imperatore pagano; e non 125 desiderava la morte del figlio perchè aveva penetrato l'arcano che, se vivesse, diventerebbe imperatore, ed esalterebbe la santa Chiesa, l'allieterebbe e reprimerebbe in essa ogni eresia, esaltandola.

In quel mentre il Signore ordino la morte del padre di lui, affinchè Costantino dalla dottrina di esso non ricevesse danno: mando percio il Signore un angelo per togliergli l'anima; allora la fedele (Imperatrice) si rallegrò di quel ch'era avvenuto. A quel tempo vennero i dignitarî, i grandi, e le schiere di nobili, magnati e principi per seppellire l'Imperatore che da loro era trapassato. Essi pensavano che la fedele (Imperatrice) si dolesse, e non la sapevano lieta di quel ch' era avvenuto, nè che essa aveva bramato e desiderato ciò che era accaduto. Ella colla bocca piangeva, ma nel fondo del cuore assai si rallegrava: le lagrime aveva agli occhi ma

(') M aggiunge: aprì la bocca per benedire colui che forma i bambini nel ventre, e gli sug-"; gella e forma secondo la sua volontà.

(') M inserisce: che può essere quest' ulcere ostinato che non è possibile guarire: che resiste ad erbe e medicine, e che esse sono impotenti a risanare? Esso ancora rimane e noi non sappiamo che fare.

(3) $\mathrm{M}$ in luogo di 111-112 porta: « e partirono per passare da quel luogo ad un altro in fretta per salvarsi dall' Imperatore il quale come un leone gl'inseguiva senza pietà ». 
nella mente rendeva gloria grande a Dio. Essendo compinti i giorni del lutto per l'Imperatore, giunse il momento per Costantino di dovergli succedere. Alcuni dicevano che il regno gli era dovuto: altri dicevano che non accetterebbero un imperatore lebbroso; ma, poichè lo sosteneva il braccio del Signore; essi non gli recarono danno e successe al suo padre nel regno. Quando fu fatto imperatore Costantino, la terra giubilo, le creature si rallegrarono ed i mari esultarono. Fu innalzato nel

150 regno Costantino e quei che avvicinavansi gli si prostravano d'innanzi a malincuore: volendolo o no lo veneravano, mentre dalla lebbra della sua persona erano nauseati. Il puzzo della lebbra fetido com'era li metteva in fuga, e coloro che si avvicinavano con doppio cuore lo veneravano, poichè a lui dinnanzi tacevano, ma partiti si sollevavano contro lui dicendo: "Guardate! veneriamo con grande timore un imperatore lebbroso! » Si avvide l'Imperatore di tutto cio che accadeva, riguardo se stesso e non seppe che dire. «Ecco che il mio padre ha neciso i medici ed i « dotti, e non havvi un solo medico che presso di me non abbia chiamato, ma non * ho ricevuto giovamento alcuno. Che cosa faro contro questo flagello della lebbra, « che mi affligge, e contro il segno odioso che è sulla mia fronte che mi dà dolore? « Oh! se almeno la lebbra fosse in qualche parte nascosta del corpo! ma manifesta « come una stella mi sta davanti, è il discorso delle mie labbra che contamina « tutta l'aria e il puzzo della lebbra che mette in fuga chiunque a me s'arvicina. * Qual mai peccato ho io commesso che fin dal ventre materno di lebbra io sia per« cosso? Ovvero che cosa ho fatto contro gli Dei che essi abbiano a tormentare la « mia vita? Forse che mio padre non ha offerto sacrificî e aromi eletti, o che non « abbia celebrato per essi feste od olocausti? Non vi sono medici i quali possano « risanare questa odiosa lebbra: alcuni sono fuggiti, alcuni furono uccisi ed alcuni

« lapidati ». L' Imperatore avendo detto queste cose, deliberd di mandare all' istante

$1 \%$ nelle (varie) regioni: «Forse havvi qualche medico il quale scampo dal mio padre e non gustò la morte: incantatore 0 caldeo che sia qui venga ».

Percorrendo le provincie quei che furono mandati andarono e giunsero a Babilonia, la madre delle incantazioni, ed ivi narrarono l'affare per il qualè erano stati mandati, per causa, cioè, della lebbra del grande imperatore Costantino. Alcuni di essi sentirono dagli incantatori e dai caldei ('), che cioè : «Qui sonvi uomini periti « nell'arte, abili e molto versati nell' arte salutare ». Annunziarono all' Imperatore che, se egli volesse, verrebbero questi presso lui e senza ambagi monderebbero la lebbra dalle sue membra. Tosto giunse all'Imperatore la lettera che era stata mandata dagli inviati ch'erano partiti. L'aprì, la lesse e vi trovò scritto così: «Ecco, « abbiamo viaggiato nelle (varie) provincie come ci hai comandato: arrivammo a * Babilonia madre di ogni arte delle incantazioni, ed ivi narrammo per qual causa « eravamo mandati. Siamo venuti cercando uomini molto versati nell'arte (medica) « affinchè mondino la lebbra dell' Imperatore. Risposero essi, subito che ciò ebbero « udito: noi monderemo senza ambagi la lebbra del grande imperatore, per cui

(') Giacomo fa venire gl' incantatori da Babilonia come su fosse sempre il centro del magismo, gli Atti gli fanno venire dalla Persia, e Mosè di Khorene dalla Persia e dall' India. Questa incertezza stessa sembrerebbe forse un indizio dell' origine non orientale della leggenda, 
« cagione siete stati inviati ». Scrisse e mando tosto una lettera l' Imperatore comandando che senza indugio e con ogni diligenza venissero questi nomini ('). Giunse il comando ai Babilonesi e li sollecitò; gl' incantatori pure acconsentirono e tutti unitamente si apprestarono. Entrarono innanzi all' Imperatore tutti gl' inviati, e gli 200 narrarono chiaramente tutto cio ch'era avvenuto.

" Andammo, o signore, a Babilonia, madre degl' incantesimi e abbiamo con* dotto degli uomini versati nell' arte medica. Comanda, o signore, che entrino « e ti espongano la loro arte, poichè sono periti e molto abili nell'arte salutare ». Comandò l' Imperatore che entrassero innanzi a lui questi uomini, gl' incantatori coi Caldei, e stettero nel suo cospetto. Aprì egli la bocca e parlo affabilmente con essi, dicendo: «Come e con che può guarirsi la lebbra della mia persona? Codesta « odiosa lebbra che con me nacque fin dal ventre materno. Avete voi il potere di « guarirmi 0 no? Dite la verità : non desidero una menzogna, poichè innanzi alla « regia maestà, una menzogna non conviene ». Essi dissero: «Ora è sera, accordaci « tempo, o signore, fino alla mattina, che rallegra la terra col suo fulgore ». Or perchè chiesero da lui un indugio se non per imparare da Satana ogni errore? Entrarono e si nascosero a sera e presero cibo, ed in mezzo della notte invocarono il loro padre, il figlio delle tenebre, affinchè egli insegnasse loro tutta la malizia dell'arte sua, perchè in lui ogni malizia fu fin dal principio. "Vieni, o Satana, e « con te reca l'Errore, tuo padre. Questo è il tempo di muover guerra contro i fan« ciulli! Vieni, mostra prestamente la tua prodezza! che per te tutto il partito del

* male $\left({ }^{2}\right)$ sia glorificato. L' Imperatore cederà e si darà a te. Sorgi nella pugna, ed 225

« ottieni la corona del valore. Vieni subito, sta con noi e fatti a noi simile! allora

« saremo per te figli che generano prava iniquita. Sii dunque a noi padre e poniti

« alla nostra testa fortemente, e ti saremo figli che generano falsità e menzogna: ti

« ascolteremo e non ci opporremo ai tuoi comandi, perchè a noi si conviene di

« prestar obbedienza alle tue volontà. Con te venga pure il tuo compagno l'Errore « che potrà ingannare l'Imperatore col falso, e con noi venga ».

Ascolto il loro Signore, colui che insegna ogni specie di menzogna, ciò che avevano detto questi discepoli pieni d'iniquita, e si accinse tosto a compiere la loro volontà, ed a condurre a termine nel fatto, ciò che essi desideravano. Chiamò il suo padre l'Errore malvagio ed esso lo accompagnò per modo che, se mancasse qualche piccola cosa, esso vi supplirebbe. Entrò Satana accompagnato dall'Errore presso agli incantatori : aprì la bocca e parlo con loro dicendo: « Non siate tristi

(') La versione araba dice a questo punto cosi : «Quando ciò ebbe udito l'Imperatore, ne ebbe molta gioja, e mandò un suo legato a cercarli con una sua lettera, ove diceva: «Da Costantino « imperatore a tutti i medici che sono nella città di Bagdad: il nostro saluto inviamo a voi. Dacchè « ci è pervenuta una lettera da parte dei medici, i quali vi dicono che essi posson fare ciò che in « riguardo di noi hanno udito; se la cosa è vera, che si presentino prestissimamente: saranno bene « accolti e regalati di molti doni; che vengano dunque senza tardare ».

Questo è un esempio del modo in cui la versione araba ha amplificato in molti luoghi il testo siriaco.

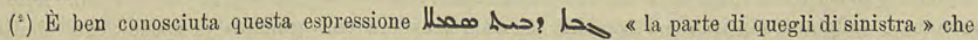
si riferise alle parole di Nostro Signore quando parla del giorno del giudizio (Matt. c. XXV, 31-46). 
« quando entrerete presso l'Imperatore, perchè io sarò con voi e lo commuoverò « con gran timore. Parlate avanti all' Imperatore e giurate, e lasciando ogni timore « sia ferma la vostra mente ». Parlo l'Errore ai figli di ogni menzogna: « Io sarò 250 « il vostro corroboratore, fatevi animo! siete miei figli e miei discepoli fin dal « principio: percio non siate perturbati, o incantatori e caldei, figli che da tanto « tempo ho allevato in perversa iniquita. Che non siano intimorite le vostre « menti per qualsivoglia modo. Io sarò con voi perchè mi avete mostrato amore $\mathrm{e}$ « benevolenza e v'insegnerò ciò che dovete dire avanti l'Imperatore. Ecco dunque che « entrate innanzi all' Imperatore e sarete interrogati: rispondete allora sulla gua« rigione e non temete. Ditegli così : Manda a radunare le primipare ( $\left.{ }^{1}\right)$, e i loro « fanciulli con esse vengano senza indugio. Manda a radunare quattro mila figli «puri di primipare: fatti un lavacro di rame $\left(^{\circledR}\right)$, uccidili, e bagnati nel sangue di « questi $\left(^{3}\right.$ ) fanciulli e sarai purificato». Tutto cio insegnò l'Errore agli incantatori, ed addottrinò in furberia i caldei: "Ora vi ho insegnato come dovete parlare innanzi " all' Imperatore * : non titubate nella vostra mente, o discepoli miei, ma ralle« gratevi con me di questa strage ed effusione di sangue e del terrore che deve " percuotere le madri. La terra tremi ed i monti si commuovano dalle loro fonda« menta per cagione del sangue che, con grande sciagura, deve versarsi sulla terra. « Le madri si lamentano con afflizione pei loro figli quando vengono di orribile « morte uccisi, il che fa la mia gioja. Uccisi questi si aftliggeranno i buoni, ma 275 « i malvagi coi quali voi siete se ne rallegreranno. Nel cielo il sole si oscurerà « per la strage dei fanciulli, e le madri afflitte si lamenteranno quando essi « saranno sgozzati» ('). Tutto cio disse l'Errore agli incantatori, ed essi ne furono istruiti, e si rallegrarono di ciò che esso gli aveva insegnato. Giunse la mattina e l'Imperatore siedè sul suo trono, ed al suo cospetto vengono tutti i grandi ed i magnati. Allora mando l' Imperatore a chiamare questi incantatori e caldei, ed entrarono e si prostrarono a lui dinanzi.

Aprì egli la bocca e parlò con essi, dicendo: «Ditemi come e con che posso « essere sanato, e, se la vostra arte ha il potere (di farlo), annunziatemelo. Non na«scondete da me ciò che sapete e di cui siete periti». Risposero essi : « Ti dicemmo

(1) La parola IA oza tanto spesso usata in questa Omelia, non trovasi nei vocabolari sinora pubblicati: però non havvi dubbio, io credo, che debbasi tradurre «primipare »: cfr. Castelli, Lex. Syr. p. 339, lin. 17-18.

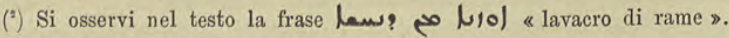

(3) Si osservi (a in luogo di (a)

(") In M invece dei versi 267 a 277 leggiamo : «domani saravvi una commozione talmente grande che non se n'è veduta una simile: i monti tremeranno, i mari si commuoveranno, e la terra si rallegrerà a causa della perturbazione che percuoterà i primogeniti delle madri; sopra esse domani cadrà gran timore; il loro seno tremerà, s'indeboliranno i loro ginocchi: domani esse si lamenteranno con grande angoscia pei loro figli, quando verranno colla spada veloce trucidati. Domani saranno angustiati tutti i bnoni, ma i malvagi coi quali voi siete si rallegreranno. Domani il lieto sole spargerà lagrime quando vedrà spargersi il sangue dei fanciulli. Domani fuggirà il gran sole, verranno le tenebre, e nube e caligine cuopriranno i fanciulli. Domani scenderà una grandine di spade acute, simile alle fiamme che uscirono dalle viti, per frangerli. 
« fin dalla sera che eravamo versati nell'arte e nell' intendimento. (Ora) manda a ra-

" dunare quattro mila madri, e che i loro figli con esse senza indugio vengano. Fatti poi « un lavacro di bronzo, e uccidi i fanciulli, bagnati nel loro sangue e sarai risanato ». (Così) parlarono innanzi all' Imperatore e diedero la destra, come l'aveva insegnato colui che gli aveva istruiti. Scrisse una lettera e la mandò alle varie regioni affinchè radunassero tutti i fanciulli delle primipare ('). In testa delle lettere che furono mandate era scritto così. «Da parte dell'Imperatore salute alle città ed ai magnati. Poscia: 300 « per queste. lettere vi fo sapere che devo compire oggi un voto solenne verso tutti « i figli primogeniti delle madri. Che ognuno di voi faccia venire tosto mila fanciulli « e li mandi per ricevere il dono che loro sarà dato ».

Aprirono i giudici e lessero queste lettere che erano state in fretta a loro mandate dall' Imperatore, e con ogni cura radunò ognuno di loro i mila fanciulli insieme alle loro madri primipare. Poscia entrarono le madri coi loro figli avanti ai giudici, non conoscendo (la causa), tutte tremanti. I giudici allora aprirono la lettera del grand' Imperatore e fecero loro sapere che non dovevano turbarsi dall'esser state radunate. "Poichè l'Imperatore ha fatto un voto solenne di farvi doni: andate « in fretta alla città capitale col cuore lieto. » Deh! quanto triste e crudele è la perfidia che l'Imperatore preparava pei loro figliuoli. Si sparse la notizia intorno alle primipare ed ai loro figli, e cadde timore nella mente del prefetto degli schiavi $\left({ }^{2}\right)$ l'ottimo e glorioso servo si preparo a lottare pugnando con Satana: si appresto

(') M aggiunge: «quattro nomini furono inviati, ciascuno ad una contrada, affinchè ognuno di loro conducesse all'Imperatore mille fanciulli. Egli scrisse una lettera ai governatori delle contrade e delle provincie, che per lui preparassero tutti i figli delle primipare.

(') Non segue bene a questo punto il nesso del racconto: il prefetto degli schiavi del quale fin'ora non si è fatto parola, viene introdotto in iscena senza ragione apparente e senza spiegazione; egli è quasi certo che debba essere stato omesso a questo luogo per negligenza del copista una parte del testo, parte che ci sarebbe stata sopplita dal M, come in altri luoghi spesso accade, 'se non mancasse qui appunto per disgrazia un foglio del codice: ma se non possiamo supplire le parole mancanti, possiarno almeno dare il seaso del passo mancante per mezzo della versione araba che qui reca le parole seguenti (al verso 347 ); premettiamo però che, secondo questa versione, i caldei comandarono che i fanciulli da sacrificare fossero cristiani:

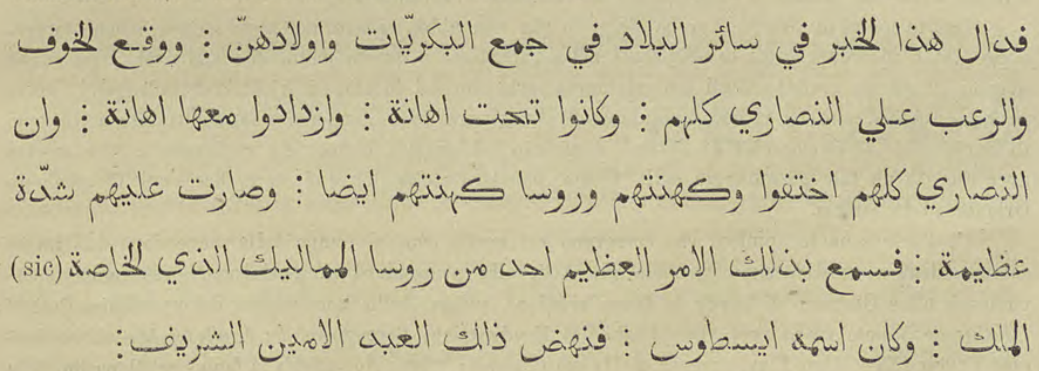

«Si sparse nelle varie provincie la notizia che dovessero essere ralunate le madri primipare ed « i loro figliuoli, e cadde timore e spavento sopra tutti i Cristiani, ed essi che erano disprezzati « n’ebbero maggior dispregio: tutti i cristiani si nascosero, ed i sacerdoti ed i sommi sacerdoti, e 
(l'animo) contro il Maligno e contro l'Imperatore, e combattè fortemente come un atleta. Nel frattanto entrarono tutte le madri innanzi all' Imperatore portando nel 325 seno i loro diletti. Il prefetto degli schiavi vide la grande bellezza di questi innocenti pargoletti, innalzò gli occhi, dalle pupille fe' scorrere lagrime, e cominciò ad invocare tristamente il Signore del cielo. «Deh! o Signore, se tal' è la tua volontà, « salva questi fanciulli. Non lascia, o Signore, che quest' Imperatore sia da Satana « sedotto! Risplenda la tua luce, si allieti la terra, e che il Maligno sia svergognato ». Scrisse una lettera il prefetto degli schiavi e la mando alla-madre fedele dell' imperatore Costantino, dicendo: «Quest' è l'ora per te di combattere contro « il Maligno, poichè il tuo figlio si prepara ad infliggere una orribile calamità alle « madri! Sorgi e prega, e non ristarti dal supplicare! Portami ajuto, ed io sarò « con te innanzi a Dio». Tutto ciò mando il prefetto degli schiavi alla madre dell' Imperatore (') affinchè ambedue facessero insieme guerra al Maligno. Subito che la prudente (Imperatrice) ebbe cio inteso, solleva in alto le pupille, versa lagrime, e prega tristamente, facendo un' orazione piena di mestizia pei fanciulli. « O Ottimo! tu che scendesti dal Padre per redimere il genere umano, abbi « pietà delle immagini che le tue mani han formate affinchè non periscano! Il « Maligno sta per infliggere una orribile calamità alle madri: deh! che si diffonda « la tua misericordia sopra i loro diletti affinchè non perdano speranza! " $0 \mathrm{~h}$ ! qual grata preghiera fu quella che fece la madre dell' Imperatore, e mando a Dio in favore dei fanciulli! Poscia scrisse e mandò al prefetto degli schiavi (dicendo): 350 « Io sarò con te in questa lotta, non turbarti! ma sorgi, o generoso, ed ottieni « la corona di tua prodezza! ed io da parte mia supplicherò il tuo Signore con « dolore e tristezza ». Ricevè il servo la lettera mandata dalla madre dell' Imperatore, e si allietò sentendo che essa sarebbe con lui avanti Iddio. Entrò il servo col cuore pieno d' un grande dolore, e si prostrò innanzi al grande Imperatore con ira estrema : alzò gli occhi e mirò gl' incantatori ed i caldei, e si fa di un aspetto pieno d' ira contro loro. Aprì la bocca l'ottimo servo e parlò con lui e comincio

\& venne sopra loro una grande angustia. Udi questa grande cosa uno dei capi dei servi dell'Imperatore, « il cui nome era Giusto: e sorse questo servo fedele e nobile» ecc.

Questo nome di Giusto è probabile però che non abbia esistito nel testo siriaco, poichè la versione araba introduce tanti cambiamenti nella narrazione a questo punto che può aver fatto anche questo. Di questo prefetto degli schiavi non-si parla che nei documenti orientali della leggenda couse in Ebn-al-Athir, però ne vediamo chiaramente il confronto in un documento assai anteriore a Giacomo di Sarûg, nel Aľv́rtııs cioè di Zusimo, confronto del quale abbiamo già ragionato e che mostra aver esistito un tale personaggio nella forma popolare della leggenda che fiori nel $\mathrm{V}^{\circ}$ secolo in Oriente ed in Grecia.

(') Varie sono le opinioni che correvano nel medio evo sul tempo della conversione dell' Imperatrice Elena al cristianesimo. Secondo gli Atti, ella propendeva al giudaismo, però molti docucumenti, oltre Giacomo di Sarûg la fanno cristiana prima della conversione del suo figlio. Così il Libellus de Sancta Helena ecc., pubblicato dal Heydenreich, Berengosio, De Laude el Inventione Sanctae Crucis $\left(\mathrm{XI}^{\circ}\right)$, Ugo Flaviniacense, nella sua Cronaca; quiesti documenti la fanno cristiana fin dalla nascita. Nella prefazione ai canoni arabi del concilio di Nicea (Labbe, Concil. vol. II, col. 391 e seg.) dicesi nata a Edessa e convertita mentr'era assai giovane: cfr. Coen, Di una leggenda ecc., di Costantino Magno. Arch. Soc. Rom. di Storia Patria vol. IV, p. 11 e 12. 
ad intercedere in favore dei fanciulli, affinchè non fossero uccisi : «Non muoiano, « o Signore, questi pargoletti, se tale è la tua volonta! poichè il sangue dei fanciulli « non possiede la virtù di mondare la lebbra. Mesciamo le acque del battesimo, « scendi, e immergiti in esse, ed io ho in Dio la speranza che esso ti monderà: « non solo la lebbra delle tue membra monderà esso, ma anche l'iniquità che « le tue mani hanno fatto. Possiede questa celebrata virtù il battesimo; fa mira« coli e prodigi e meraviglie. Di cio mi è testimone la veste di Aezio il quale « era in Antiochia ('): poichè quando Simeon Pietro la ebbe fatta in dodici parti, « la pose nell'acqua e, quando la trasse fuori, era (stata) tessuta dentro le acque. «Allora credette Aezio che il battesimo rimette i peccati. Se le acque compirono « un abito tessuto dal battesimo, la tua lebbra non riescirà difficile al battesimo». Allora l'Imperatore tosto si adiro contro il prefetto degli schiavi, dicendogli : 375 «Vattene dal mio cospetto, o servo malvagio! ora conosco che tu desideri molto « il mio danno, e che non brami che io guarisca». Rispose il servo: «Ti piaccia « ascoltarmi, o Imperatore, e fatti agnello del gregge del Figlio di Dio. Ma, se « non mi ascolti, il cielo e l'abisso alzeranno contro te la voce, tanto più se verserai « il sangue dei fanciulli. Deh! che non sieno spinte le madri ad imprecarti acer« bamente! perchè il Signore nel cielo udirà la voce dei loro pianti. Se tu levi la « spada contro i fanciulli come hai deliberato, il Signore nel cielo non tollererà questa « tua grande iniquità ». Dopo che ebbe detto tutto ciò il servo si allontanò ; poichè la mano del Signoré era con lui, e non temeva. Allora la madre dell'imperatore Costantino spoglio le sue vesti e si coprì d'un saceo, e (quasi) volando uscì come colomba dal suo nido, affinchè dalla bocea del falco salvasse gl' innocenti fanciulli. Scese sollecitamente e stette d'innanzi al trono, e quando la videro i nobili ( $\left.{ }^{2}\right)$ furono spaventati. Insieme ad essa entrarono il prefetto degli schiavi e tutti gl'imprigionati con grande lamentazione innanzi all'Imperatore come narrammo. Aprì la sua bocca la beata (Imperatrice) per parlare: «Pensa quanto ti amai quando fanciullo ti educai, « e sappi che cosa amano queste donne i loro figliuoli. Per quella misericordia che 400

« ti formò nel ventre di tua madre, non fa che essa oggi si affligga pel suo figliuolo! «Per questo seno che ti allattò mentr'eri fanciullo, non si sparga il sangue puro « degl' innocenti pargoletti! poichè il sangue umano non possiede la virtù di

(') Questo passo riguardante Aezio è tratto dall'Omelia del medesimo Giacomo di Sarûg sulla conversione di Antiochia, e serve a provare l'autenticità di essa messa in dubbio dall Assemanni. Questa Omelia si legge nel nostro codice 11\%, fol. 522-526 (cfr. Assem, Catal. T. III, p. 103, 104;

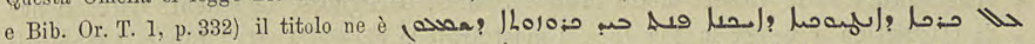
o \&ella città di Antiochia: del modo in cui fu « convertita per mezzo della predicazione di Simeone principe degli apostoli, di Giovanni apostolo « e di Paolo apostolo». Questa narrazione, tratta dagli apocrifi, narra come S. Pietro e S. Giovanni cercando di convertire gli Antiocheni furono perseguitati, come S. Paolo venne al loro ajuto fingendosi pagano e convertito dai miracoli operati in presenza di tutto il popolo da S. Pietro: poscia S. Paolo persuase a tutti di abbracciare la fede e di essere battezzati nella casa di S. Pietro mutata in chiesa. Il primo a ricevere il battesimo fu Aezio, il sommo-sacerdote dei pagani e l'istigatore della persecuzione contro i due apostoli. Egli fu pienamente convertito dal miracolo operato da S. Pietro colle acque battesimali, cui si accenna in questo passo della nostra Omelia.

() Probabilmente uno sbaglio del copista per مa " gl' incantatori 》. 
« mondare la tua lebbra ('). Non farti adunque ingannare dal discorso malvagio di « gente omicida. Che, se tu fai tutto ciò, io ti abbandonerò, ed al cospetto dei " magnati apertamente proclamerò che tu non sei mio figlio ». L'Imperatore tosto « si adirò e rimproverò la sua madre dicendole: « resta ed ascolta e non sii «turbata!» e mentre essa era presente chiese agli indorini ed ai caldei di dire avanti ad essa cio che da Satana avevano imparato. Egli aprì allora la bocca e comincio a parlare cogli indovini e coi caldei al cospetto della sua madre: «Ditemi « se il sangue dei fanciulli ha la virtù di mondare la lebbra, oppure se mi avete « pienamente ingannato ». Aprirono la bocca gli indovini ed i caldei e cominciarono a gridare audacemente avanti a tutti: «Noi conosciamo l'arte medica fin dall'anti425 « chità, e non vi sono erbe che possano mondare la lebbra da te. Di certo cono« sciamo la nostra arte nè vi ha cosa che ciò possa fare quanto il sangue dei « fanciulli primogeniti delle madri ». (Quindi) principio l' Imperatore a dire, adirato, alla sua madre: "Tu non vuoi che sia mondata la lebbra del mio corpo. Quanti « medici sono venuti da me e non mi hanno recato giovamento! ed ora questi, « essendo venuti, han cura della mia guarigione». Essa gli rispose : « Non ho nulla « a fare con te nè colla tua lebbra: e se farai tutto ciò, la tua lebbra crescerà, « e t'imprecherà questo seno che ti allattò, e questo ventre che ti portò griderà « contro te». Si avvicinarono i nobili coll'intera città e si prostrarono innanzi all'Imperatore, ed in mezzo alla folla dei magnati stava il prefetto degli schiavi. Essi l'esortavano, pregandolo così : « Ascolta la tua madre! non siano uccisi questi fanciulli! « poichè non conviene alla tua maestà di fare una tal cosa, nè che la notizia della « strage che avrai fatta si sparga per tutta la terra!» L' Imperatore si adirò contro i magnati e li rimproverò : allora sostenne il prefetto degli schiavi esso solo questa lotta. Aprì la bocea il buon servo e principiò, dicendo : «Io non ristarò dalla difesa « dei fanciulli, anche se ne muoia : non mi saranno molesti i tuoi tormenti, o Impe« ratore, per cagione dei fanciulli: per essi lotterò affinchè non vengano uccisi ». Disse l'Imperatore: « Va via dal mio cospetto come già ti dissi, altrimente io « mischiero il tuo sangue con quello dei fanciulli ». Uscì il servo, afflitto, dal cospetto del suo signore ed ando per narrare alla fedele (Imperatrice) quanto aveva udito. La vide prostrata in preghiera innanzi a Dio ed anch' egli si prostrò con grande tristezza per pregare. Al principio della loro preghiera tessero una corona 450 al. Figlio di Dio, e con parole formarono una preghiera ammirabile. «0 Ottimo che « scendesti e dimorasti nel ventre della benedetta (vergine), che si diffonda la tua « misericordia sulle viscere delle madri! Sperdi, o Signore, le tenebre dalla mente « di Costantino e venga la tua luce a mostrargli la via della vita! Libera i fanciulli « e salva l'Imperatore dall'errore; e che si allietino le madri e sia svergognato il « Maligno ». Questa soave preghiera colla fede volando sali in alto al cielo fino al misericordiosissimo Iddio: l'onorarono le legioni celesti perchè era accetta, e la

(') Era sparsa nel medio evo la credenza, che il sangue umano potesse guarire la lebbra: questo appare chiaro in varie scene di quei Misleri, tanto popolari in quei tempi (cfr. Coen op. cit. p. 14): nel Miracle de Notre Dame l'Amis et d'Amille, questo rimedio viene adoperato con buona riuscita, dietro il comando d' Iddio stesso, ed esso è consigliato da un medico nella Rappresentazione di un miracolo di due pellegrini. 
introdussero e la posero innanzi al trono della divinità : « Ti supplico, o Signore, « per quella misericordia che te fece venire a noi, salva $i$ fanciulli e converti « l'Imperatore del suo errore! Per la tua passione e la tua morte e la tua crocifissione «fu redenta la tua chiesa: vieni al suo soccorso come sei uso fare, o clemente!» La preghiera salì ed ottenne misericordia dal clemente (Iddio), e la misericordia si diffuse per salvare codesti fanciulli ('). (Iddio disse) : « 0 preghiera, torna in « pace a chi ti mando, poichè ora io invierò uno fra gli angeli e li salverà » $\left({ }^{3}\right)$. Si sparse adunque la misericordia del Figlio di Dio, come era suo costume, ed egli mandò il suo messaggero, il quale velocemente scese e, sotto la figura di un giovinetto venne umilmente a lui (l'Imperatore) d' innanzi, per non mostrargli la veemenza del suo ardore $\left({ }^{3}\right)$. Quando vide l'Imperatore questo giovinetto, rimase commosso e stupefatto e chiamò il prefetto degli schiavi ed a lui parlo con gran timore e tremito dicendo: «Ecco, io veggo un uomo di fuoco molto terribile, che tiene uno 475 « scettro di fiamma e vuol percuotermi. Vieni! avvicinati a lui, se lo puoi, e « dimandagli di dove egli è, e che cosa desidera, perchè esso è molto terribile!" (') Si avvicinò il prefetto degli schiavi e si prostrò ed adorò con gran timore * innanzi a quest' nomo di fuoco rivestito di fiamma $\left({ }^{5}\right)$ : « Dimmi, o signore, di dove sei e « qual'è la tua dimora, poichè avanti a te trema il diadema (la regia maestà) « dell' imperatore Costantino ». Rispose egli : « Io sono uno degli angeli che il «Signore ha mandato per salvare i fanciulli. Di' percio all' Imperatore che non « siano uccisi oggi questi pargoletti per timore che, per il suo delitto, non si scon« volgano la terra, il cielo e l'abisso ». Mentre principiava il servo a ripetere all' Imperatore queste parole e manifestargli cio che dall' angelo di fuoco aveva udito, * s'innalzò l'angelo dal cospetto dell' Imperatore. Allora l'Imperatore domando al prefetto degli schiavi che fare; ma quegli innalzava lode ed azioni di grazia perchè il Signore aveva ascoltata la loro preghiera ed esaudita la loro domanda. Rispose (poi) all' Imperatore il prefetto degli schiavi dandogli così consiglio : « Ascol« tami, o Imperatore, perchè io molto bramo la tua guarigione! $\left(^{6}\right)$ Che restino i « fanciulli in prigione come stanno: sia consecrato il battesimo, o signore, scendi « ed immergiti in esso ed ho speranza in Dio che esso (battesimo) ti monderà e « che la tua lebbra odiosa che ti affligge cesserà. Se dalle acque del battesimo esci

(1) In $M$ invece dei versi 460 a 465 leggiamo: « vidde (Iddio) ch'era afflitta e triste a causa dei fanciulli e fu spinto a salvarli egli stesso ».

( $)$ M: «Quel giorno apparve a Costantino una visione terribile che lo convertì dall'errore della via ch'egli aveva tenuto ».

(3) Let: « della sua fiamma».

(') Secondo M l'Imperatore dice: «Ecco io veggo qui un giovane che non è di noi (di nostra razza): il suo aspetto è bello, piacevole la sua apparenza: egli tiene uno scettro di fiamma e sta alla mia testa, e mi sembra, secondo quel che penso, ch'ei roglia percuotermi. Avvicinati e dimandagli di dove egli è, se lo puoi, e non darmi falsa relazione di alcuna cosa che dalle sue labbra udirai.

(s) M dice: «tutto tremante poichè non sapeva con chi avesse a fare: e principiò il buon servo a parlare in questo modo innanzi all'Angelo ».

( $\left.{ }^{6}\right)$ A questi versi (489-494) M sostituisce gli altri: «se mi ascolti, o buon Imperatore, io ti consiglierò e ti preparerò a respingere l'inferno (?) ed ho speranza in Dio che a tuo giovamento riuscirà ciò che ora ti dico, seppure mi ascolti ». 
500 « guarito (bene); altrimenti ecco, i fanciulli stanno nella prigione; che sia fatta « la tua volontì ». Rispose l' Imperatore: « Accetto questo tuo consiglio ed il tuo « discorso mi è grato, o servo, e lo amo ». Con gioia estrema corse il prefetto degli schiavi, latore di belle speranze, alla madre dell' imperatore Costantino: « Ha «brillato la luce nella mente di Costantino, le tenebre ne sono seacciate ed il « Maligno e l'Errore insieme a lui sono svergognati ». Quando udì la madre di Costantino le parole del servo, stese le mani ed aprì la bocca per render gloria a Dio : « Ti adoro, o Ottimo, che mostrasti all' Imperatore la tua luce e lo chiamasti « e l'avvicinasti al fonte del battesimo». Corse quindi il prefetto degli schiavi alla prigione stessa per dare alle madri speranza di salvezza, (dicendo loro): « ì sorta « la luce (di salvezza) sopra i vostri figliuoli; non attristatevi! * poichè il Signore « ha udito nel cielo dei cieli la voce dei vostri gemiti ». Risuono colà una nuova lode con rendimento di grazia a Dio dalle bocche delle madri ad alta voce ( $\left.{ }^{1}\right)$. «Sia « resa gloria a te, o Figlio di Dio, che dal principio alla fine cerchi di salvare gli « uomini. Noi ti adoriamo, o Figlio, che sei disceso pel tuo grande amore e sal« vasti gli uomini dall'errore (idolatria) di demonî immondi. Sii benedetto, o Signore, « per aver udito là preghiera della madre dell' Imperatore, * e si è placato per le « tue preghiere (o servo), ed ha salvato i nostri figliuoli. Il Signore ti benedirà, 0 « giovane apportatore di liete speranze, ed anche le generazioni che verranno dopo 525 « si ricorderanno di te $\left(^{2}\right)$. Comando quindi l'Imperatore al prefetto degli schiavi senza ritardo (dicendo): Fa ciò che vuoi! va ed apparecchia il battesimo! » Ricevè il servo la lettera venuta dall' Imperatore e con grande gioja e letizia gli si prostrò d'innanzi; poscia diresse velocemente il suo cammino alla prigione per annunziare ai fanciulli la lieta notizia della loro salvezza, e così loro fu detto dal prefetto degli schiavi : * Sorgete a pregare ed a supplicare innanzi a Dio! Costantino va per esser « battezzato, rallegratevi o fanciulli, perchè dai Caldei che vi minacciavano io farò « vendetta! » 0 il messaggero che porta pace ad ambedue le parti, e che somiglia a Gabriele Arcangelo! Porta l'arcangelo il messaggio a (Maria) l'ebrea e porta il servo il saluto al (sommo) sacerdote' da parte dell' Imperatore. L'areangelo così parlò a Maria: «Salute, o Maria, il Signore è con te e da te nascerà! » ed il prefetto degli schiavi disse cosi al vescovo: «Salute, o sacerdote, Costantino viene per esser «battezzato». Il (messaggero) celeste spargeva pace fra i terrestri, ed il terrestre spargeva pace nel santuario stesso. Volò Gabriele e scese dal cielo in basso; il servo usei dal palazzo verso la chiesa santa. L'arcangelo per la sua venuta attristò la morte, ed il prefetto degli schiavi sconfisse il Maligno colla sua uscita. 0 i 550 messaggeri! quanto si somigliano l'un l'altro, il celeste ed il terrestre! cosa mirabile!

(') M: « dacchè il Signore ha udito la vostra preghiera allietatevi in pace: guardate dice quanto io ho sopportato in questa lotta e pregate per me innanzi a Dio come io ho lottato ». Aprirono la bocca le madri per lodare quell'Ottimo che fece sorgere la sua gran luce nel cuore dell'Imperatore 》 ecc.

(') In luogo di 522-524 leggiamo in $\mathrm{M}$ : « e benedetta sia colei che tanta sollicitudine ha mostrato pei nostri figlinoli. Entrò il servo avanti all' Imperatore e gli si prostro dinnanzi; aprì la bocca e con esso suavamente parlò (dicendo): «Comanda o signore Imperatore che sia per te consacrato il battesimo: scendi ed immergiti in (sso, e subito scomparirà da te la tua lebbra». 
Corse il servo per la via che conduce alla chiesa lieto e felice, adorando, magnificando, e confessando Iddio. Aprì quindi la bocca e salutò il vescovo: «Salute « a te! Costantino viene per esser battezzato. Sorgi e consacra senza indugio il « battesimo! perchè l'Imperatore viene per esser da te battezzato; e distruggi « l'Errore. Ecco che tutti i malvagi sono attristati. Risplenda (dunque) la luce, « e si allieti la chiesa, e che canti lode al Figlio Unico (di Dio) il quale soffrì « sulla croce e diede per essa (chiesa) il suo corpo ed il suo sangue vivificante « per la remissione delle colpe ed il perdono dei peccati. Ed il battesimo che genera " figli spirituali egli in essa stabili affinchè (gli uomini) fossero eredi del regno «celeste! » Allora comando il vescovo a tutto il clero e si apparecchiarono pel rito glorioso del battesimo. Uscirono $\mathrm{i}$ banditori per tutta la città e proclamarono che cioè «l'Imperatore viene battezzato: rallegratevi tutti voi che siete tristi! »." Udì la madre dell' Imperatore che egli andava alla santa chiesa per scendere ed immergersi nel battesimo, ed innalzo lode al Signore dei re, il quale aveva esaudito la sua voce, ed aveva salvato l'Imperatore ed irradiato la sua luce nella mente di lui : « O Cristo, mio salvatore, gloria sia resa alla tua misericordia ed alla tua « bontà! La bocca non può glorificarti, o Signore, come converrebbe; conviene che " dalle tue creature tu fosti continuamente glorificato, poichè tutti tu vuoi rivificare « per la tua misericordia, come suoli » (').

Quando udirono i caldei e gl'incantatori quant' era accaduto, gridarono tutti 575 amaramente per l'angoscia: "Guai a noi che siamo stati grande calamità a questa « terra, nella quale, se gli Dei non ci aiutano, moriremo! * Guai a noi! non sap« piamo che fare! imperocchè è giunto il giorno in cui riceveremo il nostro gui« derdone, simile a ciò che abbiamo consigliato (per altri) ». Usci l'Imperatore dal palagio con grande pompa per andare a lavarsi ed essere mondato col battesimo $\left({ }^{2}\right)$. Uscì il sommo sacerdote dalla chiesa ad incontrare l'Imperatore, con tutto il clero, e colle schiere dei nobili. Si avvicino il prefetto degli schiavi innanzi all' Imperatore dicendo: «Ecco! il sommo sacerdote viene al tuo incontro, ricevilo e veneralo; inclina « la tua testa e prostrati a lui dinanzi con gran timore! poichè il vecchio dei giorni $\left({ }^{3}\right)$ « è onorato nel glorioso sacerdote». In quel giorno grande fu la meraviglia dei savii, poichè dalle due parti si sentivano le voci di lode; da una parte si gridava: «Bene«detto sia colui che ci diede la luce »: dall'altra parte: «Mostra la tua potenza

(1) Invece dei versi 567-574 troviamo in $\mathrm{M}$ : « corse il prefetto degli schiavi alla fedele (Imperatrice) per annunziarle: «Costantino viene ad esser battezzato, rallegriamoci oggi! togli il tuo « cilicio e rivesti le tue vesti ordinarie: lava le ceneri (dalla tua testa) e poniti la corona reale ». La fedele (Imperatrice) tosto ch'ebbe udito tutte queste cose, senza indugio le eseguì velocemente: la gioia le appariva sul viso e l'allegria sulla fronte; dalla sua bocca usciva lode a Dio e dalle sue labbra un'azione di grazie ad alta voce. Corse il prefetto degli schiavi per annunziare a Costantino che cioè: «Il battesimo è consacrato, sorgi e vieni! » Quando udirono ecc.

$\left({ }^{2}\right)$ In M leggiamo (579-582): «questo malvaggio di noi si ride, e quel che gli è piaciuto ha « narrato di noi: a noi sembra che sia giunto il giorno in cui riceveremo il nostro guiderdone ». Subito allora corse velocemente il prefetto degli schiavi per annunziare al sommo sacerdote: « Ecco! «mentre viene Costantino a divenir battezzato ti conviene, o Signore, di venire al suo incontro, \& perchè innocente è il suo cuore ».

$\left({ }^{3}\right)$ Sembra essere un'allusione al passo di Daniele VII, 9, 13, 22. 
« nel battesimo! » e le turbe gridavano: «Benedetto sia colui che allietò la santa « Chiesa, e per la sua bontà salvò i fanciulli dallo sterminio ». Proseguendo l'Imperatore giunse al cospetto del sommo sacerdote, ed inclinò la testa a lui dinanzi e lo venerò con gran timore. Il sommo sacerdote ricevè con grande gioia il saluto di lui, e colà risuonò ad alta voce una novella lode a Dio. Si allietò il pastore per 600 Costantino, nuovo agnello il quale era entrato nell'ovile, ed era stato dall'errore liberato. Principio allora l' imperatore Costantino a parlare con grande trepidazione al sacerdote illustre per molte gloriose azioni della fede : « 0 signore ! molto tempo « è da che questa lebbra mi affligge, e che dal suo puzzo infetto sono tormentato « e reso immondo: io credo, o signore, che la croce luminosa ha la virtù di mon« darmi dall' impurità che mi tormenta. Deh! sorgi, preparami il battesimo affinchè « io vi scenda e m'immerga, perchè secondo quel che ho udito Cristo Signore per « questo venne ». Disse il sommo sacerdote: « È in verità nel battesimo la virtù di mondare la tua lebbra e quel segno odioso che è nelle tue membra. 0 agnello, che qui sei venuto, non sii afflitto per la tua lebbra, perchè, prima che tu sia battezzato, io per l'olio santo ti monderò ». Comando (') quindi il sacerdote che si portasse l'olio santo d' unzione, ed aprì la bocca e con lui parlò, così dicendo: «Per « quest' olio, col quale ti ungo, sarai mondato, perchè non farò scendere al batte« simo la lebbra immonda ». Comincio allora il sacerdote ad ungere l' Imperatore tutto immondo, e cadde la lebbra di lui; e co'suoi occhi egli vide il gran miracolo: aprì la bocca l'Imperatore a lodare, ad alta voce $\left({ }^{2}\right)$, quell'Ottimo il quale

(') Ai versi 597 a 612 M sostituisce: «lo, venerò l'Imperatore, ed il sommo sacerdote anch'esso si prostrò innanzi all' Imperatore. Qual gran meraviglia il vedere l'Imperatore ed il sommo sacerdote venerarsi l'un l'altro! Ognuno di loro pregava l'altro di precederlo: l'Imperatore pregava il sacerdote ch'egli prima s'inoltrasse, ed il sacerdote pregava la maestà (corona) imperiale dicendo: procedi tu, o Signore! Vidde ciò il prefetto dei servi pieno di gloriose azioni, si pose solertemente in mezzo fra $\mathrm{i}$ due, diede la mano all'Imperatore e al sacerdote e fece avanzare parimente l'imperiale maestà ed il sacerdozio. Ambedue allora si salutarono col cuore lieto ed il sacerdote accolse Costantino e si felicitò della sua conversione. Allora principiò l'Imperatore a rivolgersi al sacerdote dicendogli; O Signore può guarirsi la lebbra della mia persona? è nel battesimo la virtù di mondare la lebbra ed il segno odioso che ò nelle mie membra? Rispose il sacerdote: Non sii afflitto per questo, poichè speriamo che prima che sia battezzato io ti monderò.

A questo verso fa seguito nel codice Bodleiano un passo che non trovasi negli altri mss. e del quale diamo qui la traduzione «Entrarono ambedue nella chiesa della Madre del sommo Re, presso la madre del Re dei Re: coll'Imperatore entrò anche il sacerdote per pregarla a dissipare la sua ignoranza. Alzò la testa Costantino e guardò l'altare e vide stante la Madre del Re dei Re e due uomini alla sua destra ed alla sua sinistra. Disse l'Imperatore: chi è quella che sta sull'altare; vedo che è una donna e che la sua bellezza è dilettevole. Rispose il sacerdote: Essa è la madre del Figlio d'Iddio alla quale ti ho accusato ${ }^{*}$; $\theta$ la ragione perchè Essa viene a calcar la terra è la punizione che essa doveva infliggerti: poichè se non ti fosti convertito Essa ti avrebbe levato la corona, e ti avrebbe fatto soggetto di scherno e di derisione». Chè questo passo abbia formato parte integrale del testo è reso probabile dal fatto che lo troviamo anche nella versione Araba.

( ${ }^{3}$ ) Ai versi 620-632 M sostituisce: «Lode a quell' Ottimo che mi diede quella luce che oggi viddi; la lebbra è cacciata; il Maligno svergognato dalla gran luce; i fanciulli salvati dal sacrifizio,

* La lezione è qui oscura, e non ci da un senso che cambiando il $y$ in $\dot{a}>$ : in questo modo trovasi in accordo colla versione araba. 
gli aveva dato la luce, e mondato da lui la lebbra, e pronunzid simili parole supplicando e pregando innanzi a Dio: «Io ti rendo grazie, o Signore, per la tua « abbondante misericordia verso il nostro genere (umano), chè per la tua venuta " vivificasti Adamo e tutti i suoi figli! Rendo grazie, o Signore, per la tua bontà 625 " che sopra di me si è diffusa, tu che da immondo che io era mi hai fatto sano! « Ti rendo grazie, o Signore, di avere illuminato i miei occhi per vedere la tua « luce! Rinnego l' errore (l'idolatria) nel quale fin dal principio fui educato: tu, « sei il Signore Cristo e Dio di ogni cosa, poichè sei tu che regni su nel cielo « e giù in terra; tuoi sono il cielo e la terra e tutto ciò che contengono, nè havvi « altro Dio che te, Signore di ogni cosa ». Disse il sacerdote al fedele imperatore Costantino: "Oggi sei divenuto qual fanciullo per la tua immersione ('), ed ora sei « mondato dall' impurità che era nel tuo corpo, vieni, entra, e lavati nelle acque « vivificanti del battesimo! Vieni, ed io ti mostrerò una madre che genera nuovi « figli, e li offre al misericordiosissimo Iddio! Vieni, scendi, e rivesti le armi nelle « acque fabbricate, e con esse combatti contro l'Avversario (il Diavolo)! Vieni, « scendi, rivesti gran forza dalle acque e sii fratello del Figlio di Dio e suo « apostolo ». Il sacerdote condusse Costantino, e lo fece entrare per essere battezzato, e tutta la moltitudine, si affollava e veniva per rimirarlo.

Quando scese il fedele Imperatore al battesimo, col cuore puro pieno d'amore e di fede, alzò gli occhi e vide un fuoco che sovrastava le acque; tremò e si spaventò a questo spettacolo meraviglioso: «Qual' è il significato della fiamma che « ora vedo $\left({ }^{2}\right)$ ardendo sopra le acque? e perchè? » $\left({ }^{3}\right)$.

Allora al comando del sacerdote l'Imperatore si avvicinò per scendere e rivestire arme spirituali: tolsero la corona del regno che era sulla sua testa, e come uomo semplice scese al battesimo ( $)$; non eravi colà corona nè di re nè di giudici, imperocchè una era la corona (cioè) il regno di Dio. Scese nel lavacro del battesimo il fedele Imperatore e salì glorioso dalle acque battesimali.

Vennero uomini da ogni parte per vedere quel gran splendore del quale era rivestito uscendo dalle acque: ivi risuonò ad alta voce una gran lode a Cristo Signore che aveva salvato l'Imperatore dall' idolatria. Esultò la madre del fedele imperatore Costantino, ed innalzò azioni di grazia a colui che, per la sua misericordia le aveva risanato il figlio: « Ti rendo grazia, o Signore ottimo che a tutti dai vita, che per

ed io convertito dall'errore e vedendo la luce eterna. Cristo viene da tutti i popoli adorato ed il Maligno è coperto di vergogna: la chiesa trionfa ed il battesimo è compito ».

(') 633 e $634 \mathrm{M}$ : «Rispose il sommo sacerdote: non ti dissi che prima che fossi battezzato io monderei dalle tue membra questa odiosa lebbra? „ il B inoltre aggiunge: « e caccerei quel segno odiato che ti tormenta?»

$\left({ }^{2}\right)$ In B leggiamo «Quella fiamma sopra le acque è essa per bruciare gli nomini ? perchè venne, e per qual causa si è mostrata? spiegaci e non nasconderlo a me ». Rispose il sacerdote: «non (bisogna di certo leggere $\mathrm{N} \circ \underset{\mathrm{a}}{\longrightarrow}$, ovvero Joa $\mathrm{N}$ invece di loa come porta Overbeck) per bruciare viene questo fuoco ma per santificare e rendere perfetti gli uomini ».

$\left({ }^{3}\right)$ Abbiamo già notato l'accordo di Giacomo di Sarûg, degli Atti, e dell'iscrizione di S. Polyeucto nel narrare l'apparizione di questa fiamma al tempo del battesimo (p. 24).

(') $\mathrm{M}$ \& e come se fosse semplice fanciullo scrissero il suo nome: non proclamarono: Ecco il Signore nostro Imperatore che viene al battesimo; bensi semplicemente Costantino scende ad ess rr battezzato ». 
" tua bontà venisti ai peccatori. Lode sia a te resa, o Signore, perchè hai convertito " l' Imperatore dal suo errore (idolatria), ed hai illuminato i suoi occhi per modo « che ha veduto la tua luce e confessato il tuo nome! Grazia ti sia resa, o Si« gnore, per aver mondato la lebbra che era nel suo corpo, e dato vita alla sua « anima col battesimo che rimette i peceati! Ecco che il mio figliuolo è stato « oggi scritto nel libro della vita, e beata me che fui trovata degna di vedere questo « giorno!»(')

Disse poscia il sommo sacerdote al fedele imperatore Costantino: * Ora che « ti sei lavato e sei mondato e divenuto nuovo, vieni e ti daro il corpo ed il san« gue del Figlio di Dio che è vita e condonatore (di peccati) dei figli d'Adamo » $\left(^{2}\right)$. 675 Entrò il sommo sacerdote ed insieme a lui l' Imperatore nel santo dei santi e gli partecipò i misteri del corpo e del sangue del Figlio di Dio (lo comunicò). Fu dunque battezzato l'Imperatore e mangio e bevve il corpo ed il sangue (divino) $\left({ }^{3}\right)$, e divenne erede coi santi nel regno celeste, sano d'anima e di corpo, servo di Cristo; e sopra lui furono diffuse tutte le bellezze della santità $\left({ }^{4}\right)$. * Uscì quindi l'Imperatore dalla chiesa con molte laudi, dotato di forza e splendore grande. Cavalè̀ l' Imperatore ed insieme a lui il pastore, padre venerabile, e diressero la loro via verso il palagio imperiale, mentre gli angeli esultavano e la turba prorompeva in suoni di lode a Cristo Signore che convertì l' Imperatore dall' errore (idolatria). Giunsero al palagio l' Imperatore, il pastore ed il prefetto degli schiavi, rendendo ogni lode al re dei re. Allora comando l'Imperatore al prefetto degli schiavi e lo fece sedere alla sua destra alla tavola al lato del sommo sacerdote; e queste parole furono dette dall'Imperatore al prefetto degli schiavi, illustre campione della fede: «D'ora « innanzi tu terrai il secondo posto nel regno, mio fratello e compagno, ed oltre « te non ne arrò altri; poichè non posso ricompensarti per l' amore che mi hai « mostrato, giacchè per tua opera mi è stata largita una nuova vita; tu mi hai « condotto nella via della vita, e con che te ne potro ricompensare? In luogo di

(') Secondo i codici M e B queste furono le parole dell'Imperatrice: «disse la sua madre: soffrite ch'io veda un poco il mio figlio unico, perchè desidero ardentemente vederlo ora che è battezzato ». Essi le risposero: «O Imperatrice, voi lo vedete tutti i giorni della vostra vita; permetteteci ora di contemplare l' Imperatore battezzato ». E quando furono compiti tutti i misteri del battesimo, e che un solo ancora mancava e non più, disse il sacerdote ecc.

(2) M porta: «Entra ora nella stanza del Unigenito, e vieni, godi del banchetto del Figlio di Dio. Vieni ti mostrerò il toro pingue che è sacrificato ed ucciso, ed ha dato a noi il suo corpo affinchè per esso si perdonasse ai figliuoli d'Adamo ».

(') M inserisce: «del banchetto del figlio di Dio ed insieme a lui il sacerdote ».

(') Tutto il racconto che segue, fino al termine dell'Omelia, è del tutto particolare all'autore e non trova confronto in qualsiasi altra versione della leggenda. Negli Atti vi è in sua vece il racconto del Concilio romano, delle leggi di Costantino, e della disputa di S. Silvestro.

Farò qui alcune osservazioni sopra qualche errore che mi sembra scorgere nell'edizione del cod. Bod. di Overbeck: la correzione di alcuni di essi ci è resa facile dal cod. M. (Brit. Mus.): per esempio a p. 356 di Overbeck, 1. 10 per $>$ deggi leggi Lم:9, 1.18 per

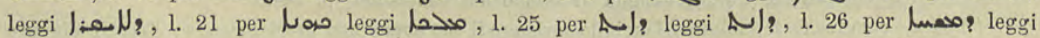

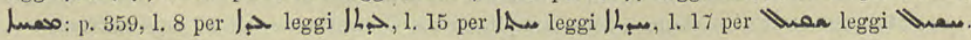


«padre a me nel regno sarai riputato» ('). Disse il servo: «Cristo Signore, che è « state teco benigno, sia con te, ed in te dimori, secondo la sua volontà! Conviene, 700

« o Signore, che quei fanciulli i quali stanno in prigione si allietino oggi in mezzo

« a questa gioia spirituale ». L' Imperatore rispose: «A te ho dato la podestà, tutto cio

« che tu vuoi fa subitamente secondo la tua volontà ». Sorse il prefetto degli schiavi, aprì il tesoro imperiale e lo distribù̀ alle madri ed ai loro figliuoli : uscirono poi i fanciulli e le madri dal palagio con grande gioia, perchè erano stati salvati dalla perdizione, benedicendo Costantino ed il prefetto degli schiavi e rendendo gloria al Figlio di Dio il quale aveva liberato i loro figliuoli. * Poscia si avvicinò il servo e venerò l' Imperatore, novello fedele, e lo supplico di prendere vendetta degl'incantatori. Rispose l'Imperatore: «Fa la tua volontà, o padre benedetto, perchè son « degni di perire senza impedimento codesti incantatori ». $\left({ }^{2}\right)$.

Scese allora il servo dal palazzo pieno d' ira e di minaccie, e lo accompagnava una folla di nobili da ogni parte, ed era onorato come re il servo fedele che da Dio misericordioso aveva ricevuto il potere. Fu posto un trono e vi sedè il servo, circondandolo i nobili, e su lui fu versata la grazia dell' apostolato. Comando il servo che entrassero innanzi a lui questi caldei ed incantatori, affinchè crudelmente gli mettesse a morte. Apri la bocea e con essi parlo dicendo con ira estrema, minacciando di ucciderli: «Non sapete voi che Gesù è re ed insieme è Dio, nè 7.5 « permette che vi sia altro Signore insieme con lui? Perchè e per qual ragione « volevate ingannare la gente del nostro paese e far trepidare i fanciulli? Non sapete « voi che il battesimo fa miracoli, che avete voluto spargere con gran terrore il « sangue dei fanciulli? Oggi stesso addurrò sopra voi una celere morte e la puni« zione della vostra audacia com' è giusto: oggi stesso il fuoco consumerà le vostre « membra, e farò patire tormenti ai vostri corpi senza indulgenza. Viva Iddio! pel « quale ho giurato santamente di non avvicinare cibo alla mia bocca finchè non « siate bruciati, farò di voi un opprobrio per le generazioni che dopo me verranno, * le quali derideranno e scherniranno la vostra magìa. Che venga ora Satana stesso « che adorate e vi salvi dall' incendio della fiamma! » Allora quelli pensarono nella loro mente dicendosi : «Parliamo con astuzia, chè forse ci salveremo; diciamo ora

(') M 681-698. « Uscirono dalla chiesa per andare al palagio l'Imperatore ed il sacerdote insieme, e lor seguiva la gente da ogni parte, ed i nobili mostrarono innanzi all'Imperatore la loro gioja ed allegria: la città esultava ed il partito delle madri allietavasi, perchè Costantino era stato mondato dalle acque, e tutte le madri che stavano in prigione udirono che il battesimo operava miracoli e portenti; esse s'inginocchiarono in preghiera ed unanimamente innalzarono la voce (lodando Iddio).

Allora comandò l'Imperatore al prefetto degli schiavi che con lui salisse nel palazzo e prendesse cibo (dicendogli): «Tu, tu sei mio padre ed oltre te non ne ho eccetto quel Padre che mi sono acquistato colle acque (battesimali): vieni, sali, riposati e prendi cibo con me ».

( ) La lezione del cod. M è qui assai preferibile (dal 711 al 714): «Disse l'Imperatore al prefetto degli schiavi: Prendi cibo: quanto tempo è da che la tua bocea non ne ha gustato. Il servo rispose: Giuro per il Signore e per la sua parola che non avvicinerò cibo alla mia bocca, come ho già detto, finchè io non abbia eseguito un terribile giudizio sopra gl'incantatori e ch'io abbia preso agli occhi di tutti una vendetta spaventevole dei caldei, perche essi han sedotto la nostra terra, e, se avessero potuto, vi avrebbero cagionato gran terrore. Rispose l'lmperatore. Così sia, come hai detto: il potere ti è concesso; fa la tua volontà prestamente ». 
« che vogliamo ricevere il battesimo e quando saremo salvati torneremo alle incan-

« tazioni ». Aprirono la bocca e parlarono col prefetto degli schiavi dicendogli:

« Voglia ascoltare le nostre parole : noi sappiamo che non havvi altro Dio che Gesù

« nè redentore nè sanatore altri che lui. Desideriamo, o Signore, ricevere il segno

750 « del battesimo ed essere numerati fra gli agnelli nell' ovile del Signore Cristo:

« crediamo ch'egli è Dio dell'alto e del profondo e che col sangue del suo lato

« egli salvò gli uomini dall'errore ». Quando ciò. udì il servo sorrise colle labbra, crolld il capo e digrignò i denti contro gl'incantatori ed i caldei : "Non bastovvi « il voler ingannare l'Imperatore che adesso cerchiate d'ingannare anche me? Io « non gitterò cose sante a cani, disse il servo, ed ai porci perle non darò. Conosco le « vostre parole bugiarde, nè potete ingannarmi come avete fatto all' Imperatore. Io « non vi permetterò di tornare a Babilonia, e non (vi) userete frodi; non offrirete « più sacrifizii immondi com'è vostro costume, nè più divinerete insieme ai divini « ed ai maghi, nè costruirete più altari ai diavoli d'ora innanzi. Io conosco la vostra « iniquità e le vostre astuzie, e sono informato della falsità dei vostri cuori. Il « fuoco saprà consumare le vostre membra: invocate Satana il vostro signore ch'egli « venga e vi salvi! » Poscia mando il prefetto degli schiavi per tutta la città che ognuno venisse a vedere il giudizio e portasse legna, ceppi di vite, canne verdi e solfo; pece, nafta e fecce d'olivo che produce gran fiamma. Vennero tutti come aveva ordinato il prefetto degli schiavi, e si prepard la folla a contemplare il ter-

775 ribile giudizio. Allora comando il prefetto degli schiavi che si conducessero in mezzo alle turbe gl' incantatori ed i caldei, con grande ira; li fece circondare di legna, nafta, solfo, pece, ed olio che dà forte fiamma. Vedendo ciò i caldei cominciarono ad invocare i loro Dei (credendo che) forse ad essi verrebbero e li salverebbero dall' incendio: «Questo spettacolo per noi è crudelmente preparato: « venite e salvateci dall' incendio della fiamma! Noi vi abbiamo adorato, ed ogni « giorno vi abbiamo servito. Questo è il momento di salvarci dall' incendio! » Disse il servo: «Gridate forte colla voce ai vostri Dei, che forse dormono e non « sentono la vostra voce» ('). Al comando del prefetto degli schiavi s'impadronirono le fiamme delle membra degli incantatori e dei ealdei: prese vendetta il fuoco terribile degli empii, ed ognuno vide la giusta condanna che a loro si conveniva. Quindi si calmò l' ira e la collera del prefetto degli schiavi, e placo il Signore per il bel giudizio ch' egli aveva decretato $\left({ }^{2}\right)$.

(') Questo passo è in imitazione delle parole di Elia ai profeti di Baal (II'-lib. dei Re, c. XVIII, v. 27). In luogo di questo verso (786) leggiamo in $\mathrm{M}$ : « o forse qualche proposito li ha portati in un altro luogo: non vi aiutano i vostri dei, lasciateli dunque ed entrate a ricevere la vostra mercede da Satana che voi adorate.

(") Nel cod. M viene inserito un passo dopo il verso 792: «Terminato il giudizio dei Caldei rimanevano gl'incantatori: comandò quindi il servo che apportassero subito dei grossi legni per schiacciarvi gl'incantatori con grande ira. Aprì la bocca e con essi parlò dicendo: ho bruciato codesti col fuoco come aveva determinato, ed ora vi farò schiacciare a voi con grossi legni. E quando ebbero ricevuto il commando coloro che stavano a lui dinnanzi, corsero e fecero premurosamente ciò ch'egli voleva. Quando il servo ebbe messo a morte gl'incantatori ed i Caldei si calmò la sua ira » ecc.

Facendo giustiziare in tempi e modi diversi gl' incantatori ed i Caldei il cod. M omette naturalmente quei versi $(787-788)$ ove il cod. Vat. parla di ambidue come cosumati dal fuoco. 
Mandò a lui l'Imperatore in riguardo del tempio ove egli adorava, (dicendo): « Che anche quel tempio ove io adorava sia col fuoco bruciato: cola io offriva « sacrifizî impuri a demonî immondi e vi ponevo aromi avanti ad immagini d'idoli « senza intelligenza.» * Rispose il buon servo al fedele Imperatore: «In questo « stesso tempio ove provocavamo il Dio dell'universo, ivi ci conviene di glorificarlo « continuamente, di adorare il suo nome, e predicare la sua santità ogni giorno. 800

« Laddove fu moltiplicata grande empietà ed iniquità infinita, colà propiziamo il « creatore dell' universo dal quale siamo redenti. Comanda, o Signore, che venga « l'illustre sacerdote, pieno di gloriose azioni, ed invochi lo Spirito Santo, e san« tifichi questo tempio, affinchè sia dimora della Divinità, Signore dei secoli, e che « sia una chiesa ove saranno rimessi tutti i nostri peccati, ed ove dimorerà la pre« senza divina (Sechina) dell'Altissimo come sul Sinai » ('). Al comando dell' Imperatore venne il pastore pieno di grazia e consacrò il tempio e vi pose l'altare santo.

Si rallegrarono i borghi e le città per Costantino, perchè il Signore lo aveva salvato dall' errore dell' Avversario (il Diavolo), e scrissero e mandarono lettere all' Imperatore fedele ove era così scritto : dicevano cioè : «Gloria sia resa all'Ottimo « che da te cacciò le tenebre dall' errore e ti fu palesata la luce gloriosa del batte« simo! Benedetto sia Cristo che mondò la tua lebbra e la tua mente (?) dall'impurità « dei sagrifizii impuri dei demonî immondi. Benedetto sia Cristo per averti fatto " agnello nel suo gregge, ed ora sei erede del regno divino. Cristo Signore sia « con te e col tuo regno, e sii terribile ai tuoi nemici ed a coloro che ti odiano! «che sii illuminato dalla luminosa croce, e che scacci le tenebre e l'idolatria

(') M dal 797 a 1809. Rispose il servo: «questo Tempio non brucierò, perchè è un gran Tempio e grande è la somma che vi fu spesa, ed esso non ha commesso nessun delitto come tu pensi, o Imperatore, perchè le pietre onde è costruito non hanno agito empiamente, ne hanno commesso iniquità le colonne ond'è sorretto: bensì siamo noi che siamo stati empii e poichè ci siamo convertiti si convertirà anch'esso. Il servo aggiunse: chiameremo il sacerdote e che lo santifichi: tosto verrà lo Spirito Santo e vi dimorerà e vi sarà la presenza divina (Sechitia), ed ivi saranno celebrate lodi alla Diviuità come furono celebrate sul monte Sinai, in mezzo alle nubi: l'olio santo puro con lui porteremo ed ungeremo il Tempio, e cosi il Signore si compiacerà di entrarvi e di abitarvi ».

$\mathrm{Si}$ confronti il paragone fatto in questo passo fra il Monte Sinai e la Basilica Lateranense (alla quale è chiaro che accenni, poichè essa fu la prima chiesa da Costantino edificata), con un'antica iscrizione che prima leggevasi nell'abside dello stesso S. Giovanni Laterano, e che qui riportiamo: la conformità dei testi non è probabilmente fortuita (Vedi Rohault de Fleury, Le Latran, p. 415).

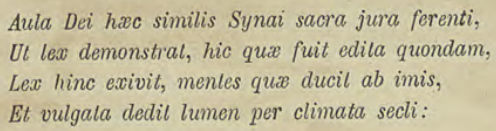

È possibile che con ciò abbia qualche relazione la tradizione che, sotto l'altare maggiore di S. Giovanni, fossero conservate le reliquie delle tavole della legge ecc., tradizione riportata in varî documenti, e nominamente nell' iscrizione presso l'altare. Il prof. I. Guidi ne ha parlato nella sua Memoria, La descrisione di Roma nei geografi arabi, p. 34, ove egli adduce altresì il cod. arab. vat. 286 che dice al fol. 107 , parlando probabilmente del Battistero Lateranense,... « l'acqua di questa fonte viene da sotto il palazzo del re (cioè il Papa) e in essa stanno nascoste le chiavi del tempio, la verga del profeta Mosè, le reliquie delle lavole della legge e l'arca, dell' alleanza: lutto ciò sta sotlo la scala ch' e ad orienle: » questo passo è di Ibn al-Vardi. 
825 « dalla terra! Ogni vittoria ed ogni aiuto ti sia accordato, perchè il Signore è stato " teco benigno secondo la sua volontà : gloria sia resa alla sua misericordia! " Ricevè Costantino queste lettere, e, quando le ebbe lette, fu riempito di gioia e d'allegrezza e cantò lode (') (a Dio), e si propose di luttare con Satana e di estirpare ed espellere le eresie dalla chiesa; e da quel tempo principio ad illustrarsi per la sua intrepidità. Sia la sua preghiera un baluardo di misericordia a tutta la nostra congregazione! $\left({ }^{2}\right)$.

\section{Fine dell'Omelia sopra Costantino imperatore.}

(') M aggiunge bene: « a quel Ottimo il quale lo aveva salvato dalle tenebri ove giaceva».

(1) Seguendo attentamente il confronto fra i due codici di Roma e di Londra possiamo formolare lopinione che quello di Londra contiene un testo assai più corretto della nostra Omelia che non quello Romano: poichè mentre le aggiunte del cod. Vat., pur uumerose che siano, non aggiungono nulla di essenziale, quelle del Brit. Mus. sembrano generalmente aver formato parte del testo originale. Questa Omelia sembra aver appartenuto ad una serie numerosa di omelie storico-leggendarie, ore Giacomo di Sarûg cercava inculcare idee e dottrine religiose e morali per mezzo dei grandi personaggi dell'Antico e del Nnovo Testamento, ch'egli prende come tipi ed esempi. È probabile ch'egli abbia composta e recitata questa serie in ordine cronologico, e ne abbiamo un'indicazione nell'esordio stesso della nostra Omelia, poichè sopra tutti i personaggi ch'egli ivi enumera in guisa da far supporre ch'egli ne abbia di già ragionato, cioè Adamo, Noè, Abramo, Asa ed Ezechia, troviamo difatti, in questa serie, una sua Omelia.

ERRATA - CORRIGE

\begin{tabular}{|c|c|c|c|c|c|}
\hline \multirow[b]{2}{*}{ Pag. } & \multicolumn{4}{|r|}{ 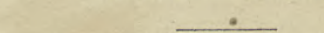 } & \multirow[b]{2}{*}{ Constantinus } \\
\hline & 27 & linea & 4 a $\mathrm{f}$. & Canstantinus & \\
\hline$\gg$ & 28 & $》$ & 16 & Breisgan & Breisgau \\
\hline$》$ & 》 & $»$ & 2 a $f$. & Einp. & Enip. \\
\hline$\gg$ & 34 & $》$ & 2 a f. & Qnesta & Questa. \\
\hline$r$ & 41 & " & 28 & $\cos a$ & così \\
\hline$\gg$ & 48 & 》 & 13 a $\mathrm{f}$. & del & dell \\
\hline 》 & 49 & » & 2 & state & stato \\
\hline$\gg$ & 51 & » & 17 & dall' & dell \\
\hline 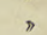 & $»$ & 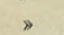 & 23 & a 1809 & all' 809 \\
\hline
\end{tabular}




\section{Aggiunta alla p. 21.}

Importantissimo è un passo della cronaca di Michele il Siro (') che non ci fu dato di poter inserire a suo posto nel capitolo sulla letteratura orientale. Nella traduzione francese di questa cronaca $\left({ }^{2}\right)$ leggiamo come, prima della battaglia con Massenzio, Costantino ebbe la visione della croce ed adotto il Labaro; come si fece cristiano e sposò Dioclezia figlia di Diocleziano; poscia vi si aggiunge: «Selon les uns «Constantin, excité par sa femme, abjura et persécuta l'Église jusqu'au moment où « le Seigneur le frappa en lui envoyant une lèpre dont il ne guérit qu'en se faisant * baptiser par Silvestre $\left({ }^{3}\right)$. Jean d'Asie attribue cette maladie à son père Constance «qui se fit chrétien et épousa Diocletia, ce qui empêcha Constantin de suivre " l'exemple de son père jusqu'au moment où, par l'apparition céleste, tous les deux «crûrent sincèrement au Christ, firent disparaître les ennemis de l'Église, et Cons«tantin resta seul autocrate».

Questo passo riveste speciale importanza, perchè non solo ci dà la forma ordinaria della leggenda, ma ci riferisce altresì l'opinione sostenuta a questo riguardo dal noto storico siro del VI secolo, Giovanni d'Asia, cio che altrimenti avremmo ignorato non essendosi conservata quella parte della sua cronica che abbracciava questo periodo $\left({ }^{+}\right)$. Ora vediamo onde Barebreo derivò questa confusione fra Costanzo e Costantino: egli non faceva che ripetere le stesse parole di Giovanni d'Asia, e percio questa variante della leggenda è antichissima e risale al tempo quando l'influenza degli atti non erasi ancora fatta sentire in Oriente.

\section{Aggiunta alla p. 26, l. 32.}

Ai musaici del portico Lateranense possiamo aggiungere gli affreschi che adornavano la loggia, ovvero «pulpitum », della medesima basilica, fatta fabbricare da Bonifacio VIII, nel 1300 , in occasione del Giubileo. T'utti convengono nell' attribuirne gli affreschi alla mano di Giotto $\left({ }^{5}\right)$. Sappiamo dal Panvinio $\left({ }^{6}\right)$ e da altri, che figuravano l'Istituzione del Giubileo, il Battesimo di Costantino, e la Costruzione della basilica Lateranense.

(1) Egli era Patriarca dei Giacobiti sulla fine del XII secolo, e la cronaca, ch'egli scrisse in siriaco, ci è conservata nella traduzione armena.

(2) Chronique de Michel le Grand, Patriarche des Syriens Jacobites, traduite sur la version arménienne du prêtre Ischôk, par Victor Langlois, Venise 1868, p. 113.

$\left.{ }^{(}\right)$In una nota il Langlois osserva: \& Sur le Bapteme de Constantin il existe un autre document dans l'ancienne litérature Arménienne dont nous parlerons dans la suite ». Di ciò non ho trovato traccia; ̀̀ lecito però di credere che alluda al trattato fra Costantino e Silvestro da una parte, e Tiridate e S. Gregorio dall' altra.

(4) Di questa cronaca si è conservato parte del secondo libro, che tratta di Giustino e Giustiniano (pubb. in Land, Anecd. T. II), e la maggior parte del terzo, che fu dato alla stampa dal Cureton nel 1853.

(5) Cfr. Müntz, Éludes sur l'hist. des Arts à Rome pendant le Moyen-Age, Boniface VIII el Giolto; p. 19-24 (Estratto dai Mélanges d'arch. et d'hist. della scuola francese di Roma).

$\left.{ }^{6}\right)$ De praecipuis urbis Romae sanctioribusque basilicis, quas septem ecclesias vulgo vocant, liber, Roma $15 \% 0$, p. 182. Panvinio attribuisce questi affreschi a Cimabue. 
- 
No.10

NOTICE

\section{SUR UN OUVRAGE ASTRONOMIQUE INÉDIT}

\section{D'IBN HAITHAM}

PAR

MA URICE STEINSCHNEIDER

EXTRAIT DU BULLETTINO DI EIBLIOGRAFIA E DI STOHAA

DELLE SCIENZE MATEA ATICHE E FISICHE

(TOMO XIV. - DICẸMBE 1881.)

$$
\text { ROME }
$$

IMPRIMERIE DES SCIENCES MATHÉMATIQUES ET PHYSIQUES

Via Lata No. 3 .

1883 


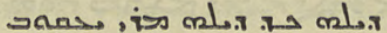

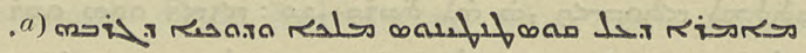

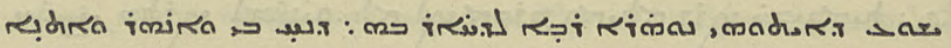

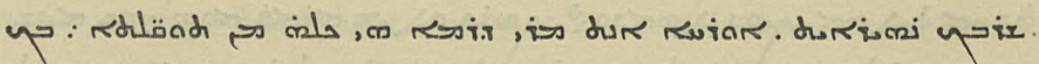

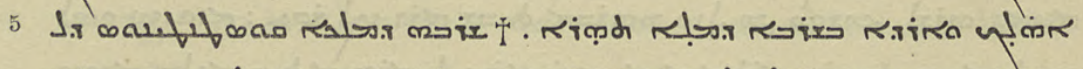
صs כis

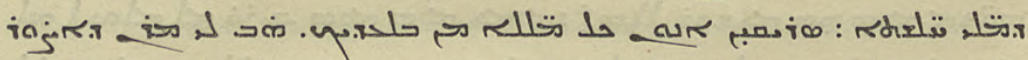

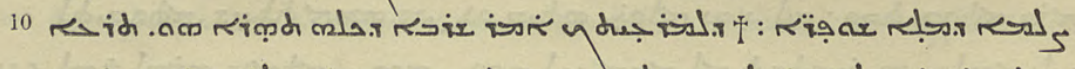
p

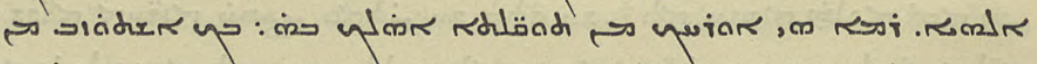

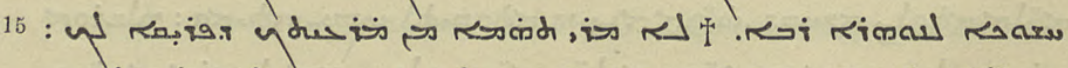

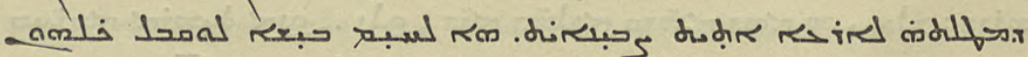

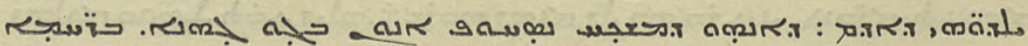

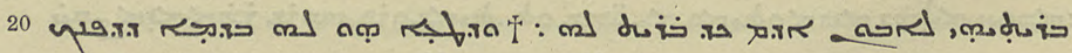

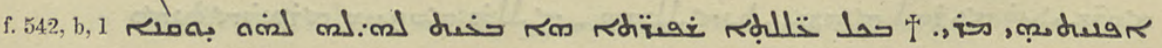

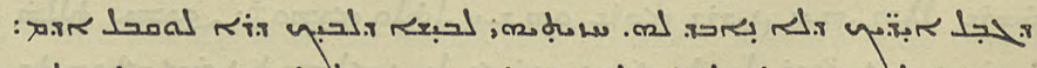

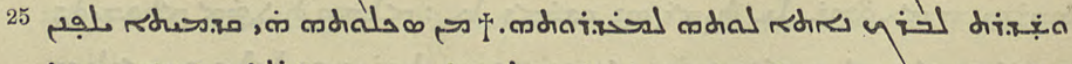

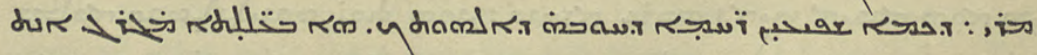
r w w

a) Il testo è quello del Codice Vaticano 117, designato colla lettera V. Le principali varianti provengono dal Codice 803 (add. 14, 588) del Brit. Mus. segnato M, e quelle che leggonsi fra i versi 495 e 686 sono tratte dal Codice Bodleiano pubblicato dal Overbeck: S. Ephraemi Syri Rabulae Balaei etc. Opera Selecta: p. 355 a 361 , e distinguonsi colla lettera $\mathrm{B}$. 


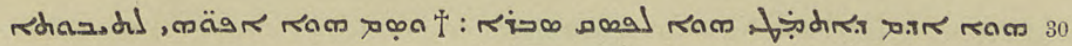

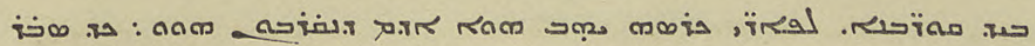

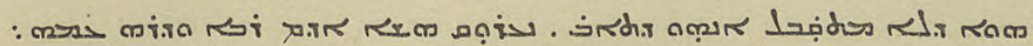

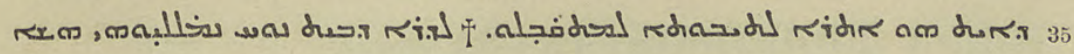

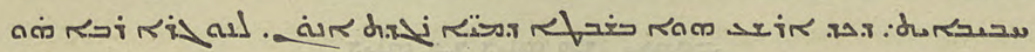

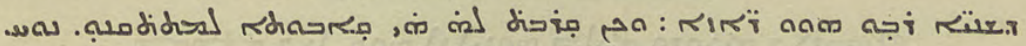

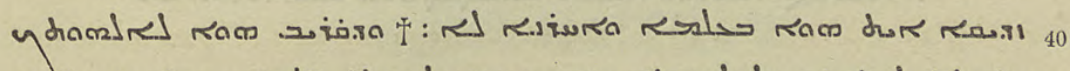

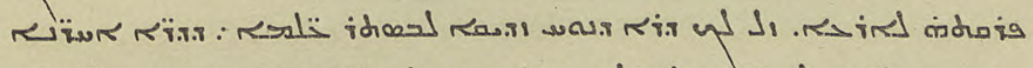
טis r. 45

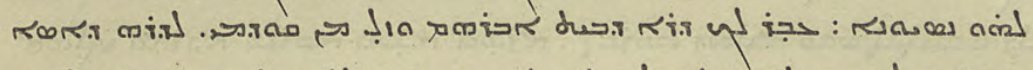

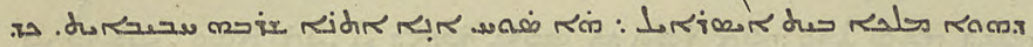

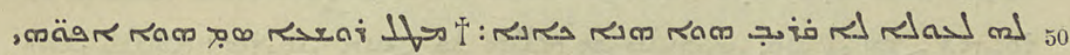

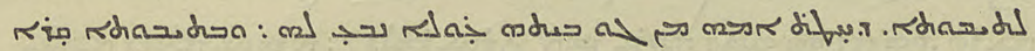

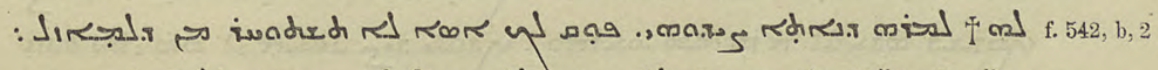

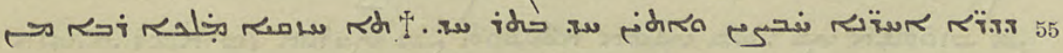

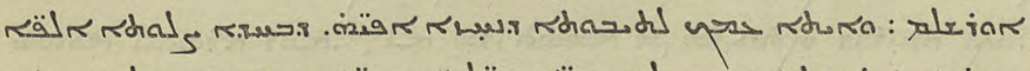

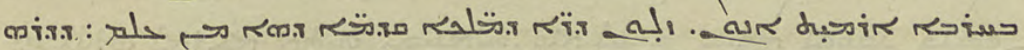

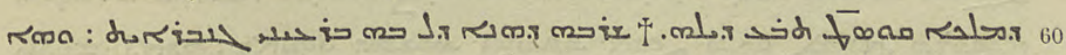

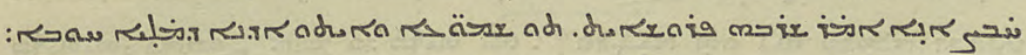

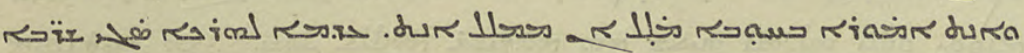

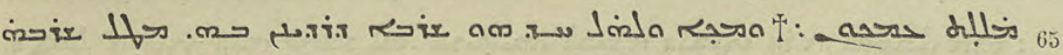

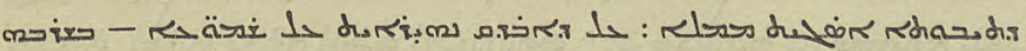

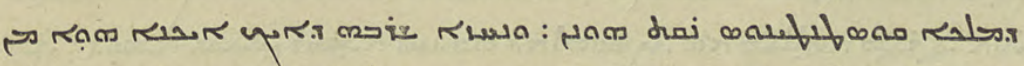

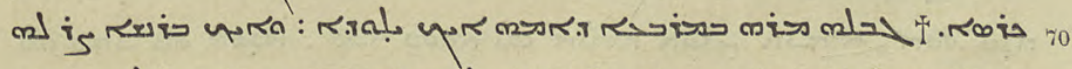
م

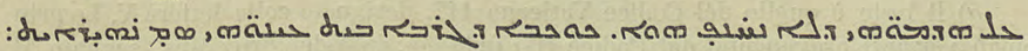

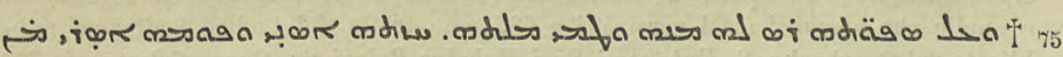

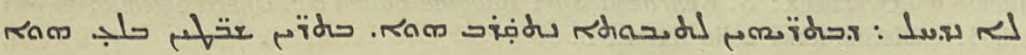




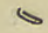

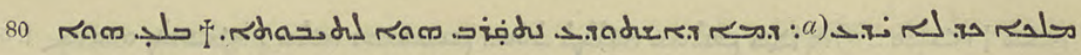

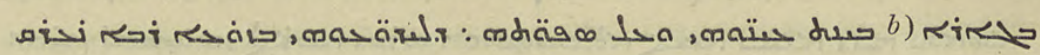

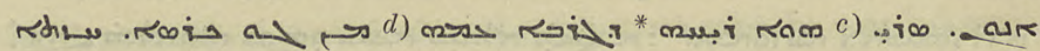

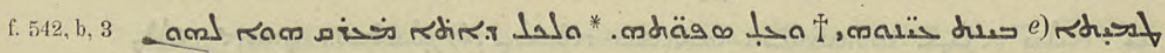

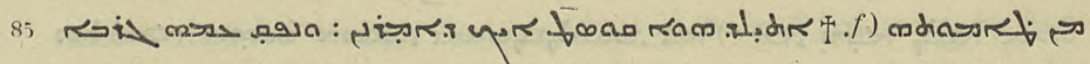

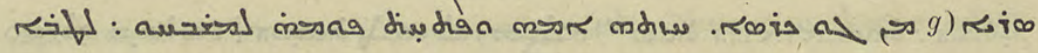
ה

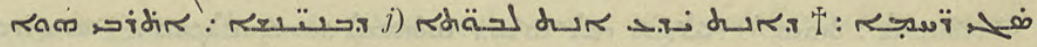

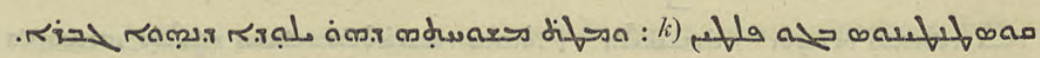
s.

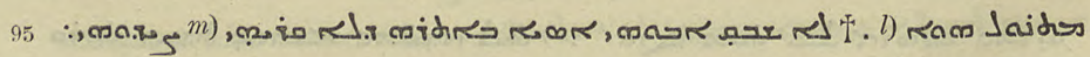

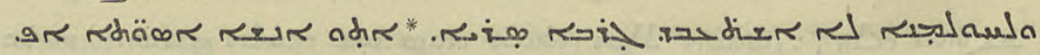

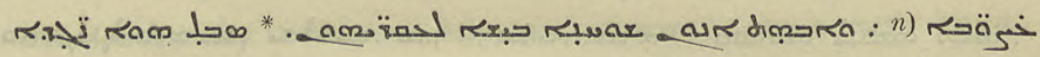

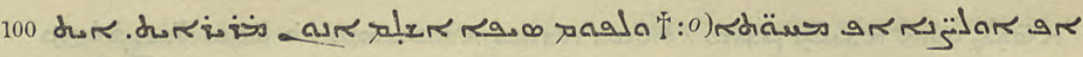
ल259

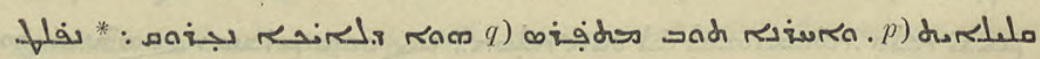

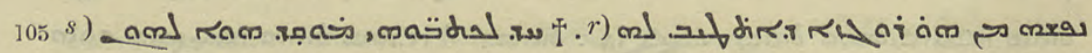

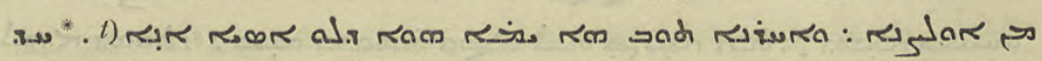
, manos Jạ Is :

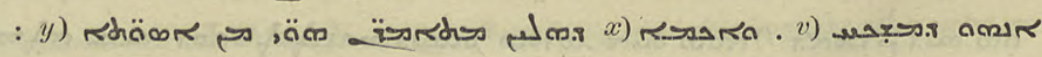

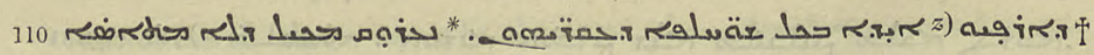

a) Il Cod. M principia alla fine del verso 78 : :Joa ...-b) M bis $-c$ ) M

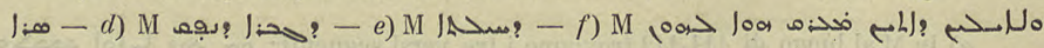

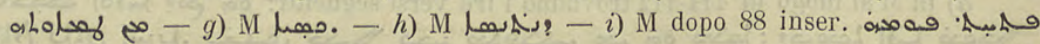

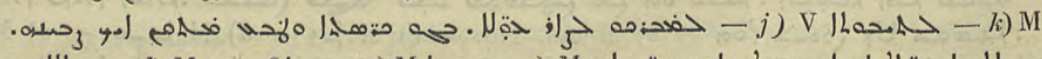

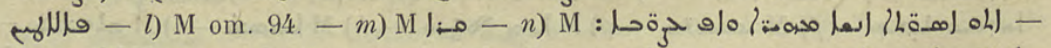

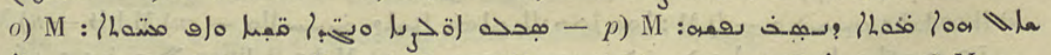
(Loحos $\rightarrow$ - - ) M x) M L L

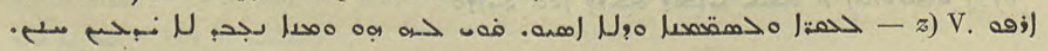




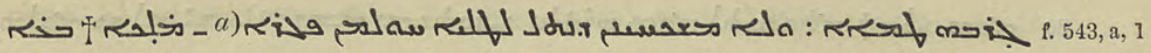

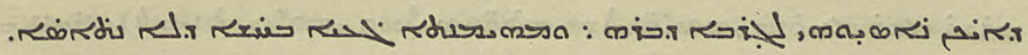

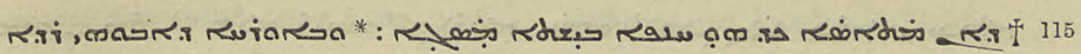

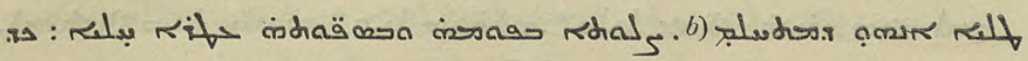

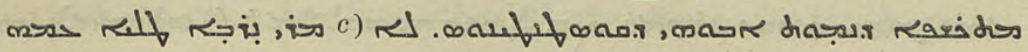
120

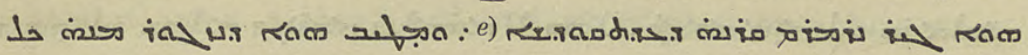

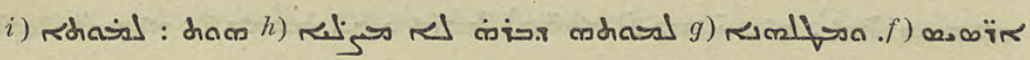

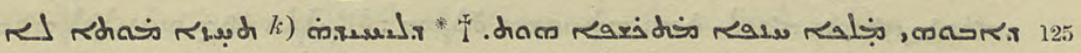

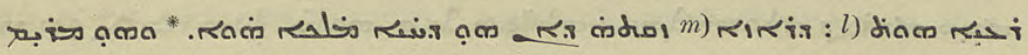

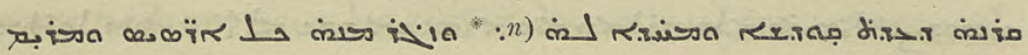
a) dies 130

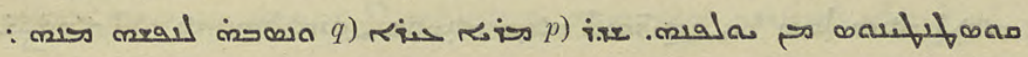

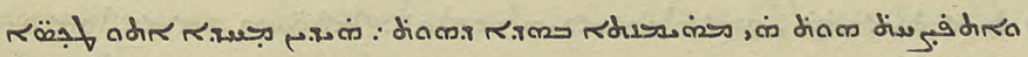
if 935

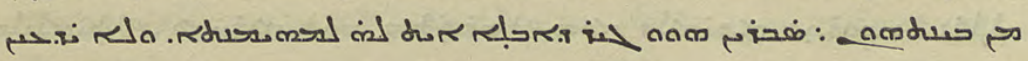

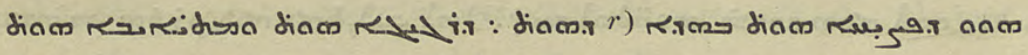

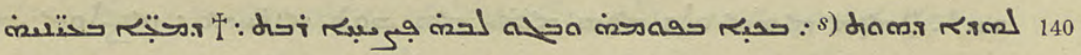

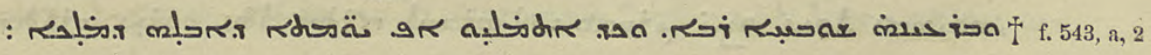

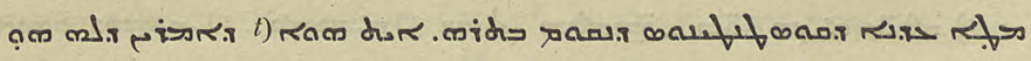

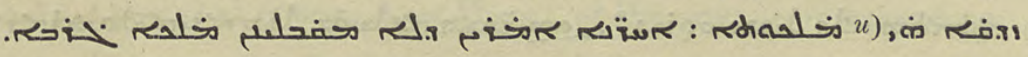
דo

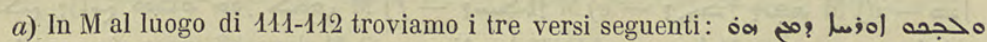

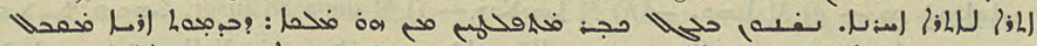

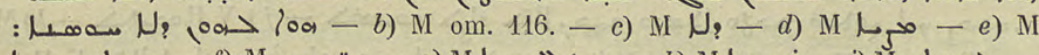

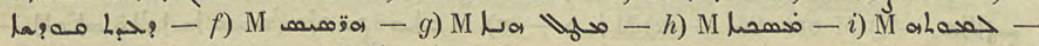
k) Il cod. ha '

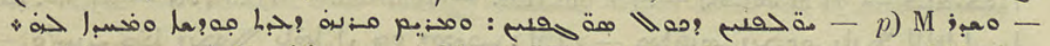

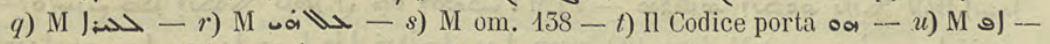

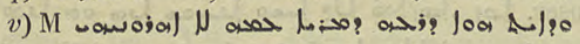




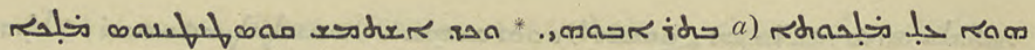

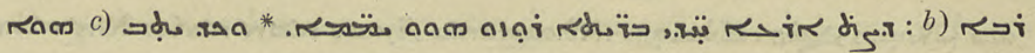

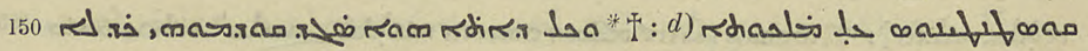

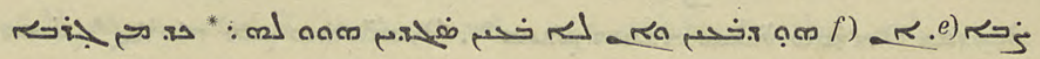

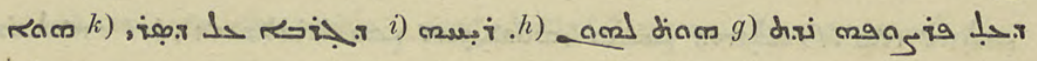

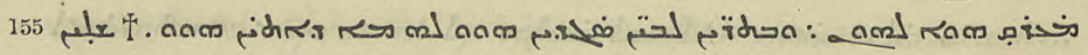

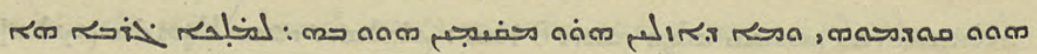

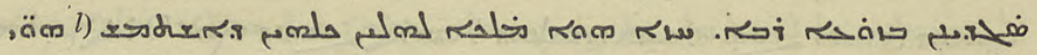

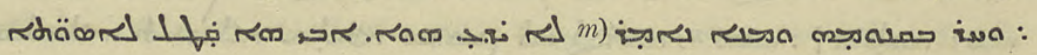

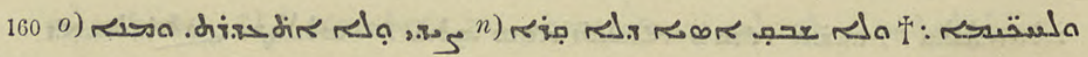

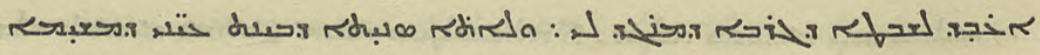

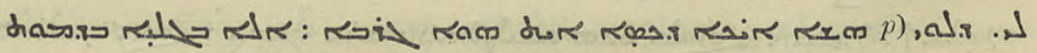

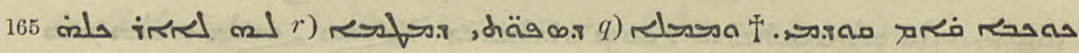

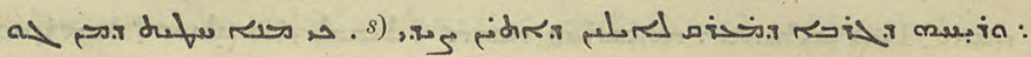

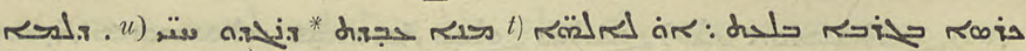

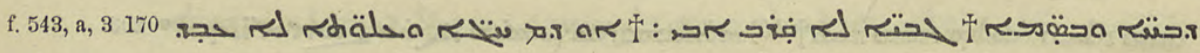

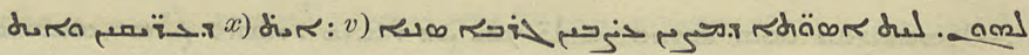

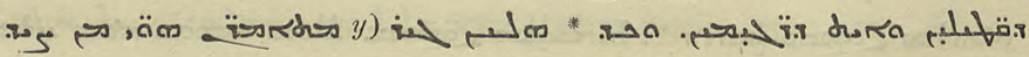
175 z) fila.

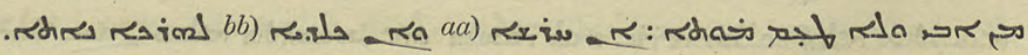

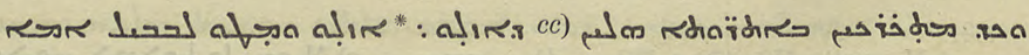

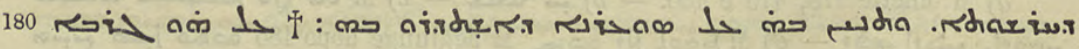

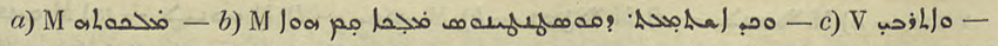

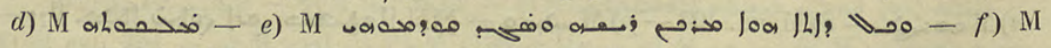

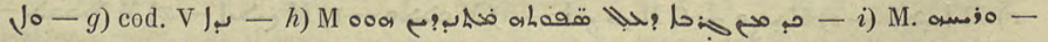

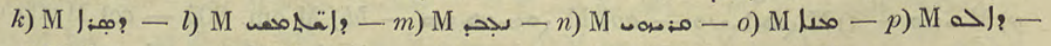

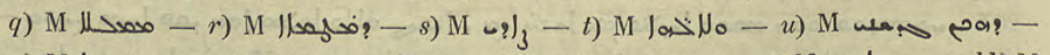

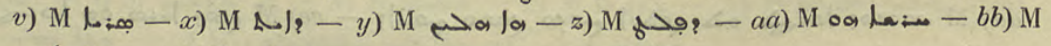
| مخدم م-cc) 


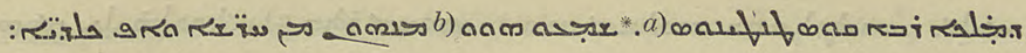

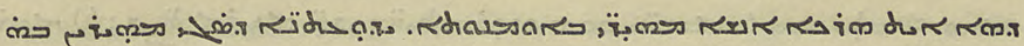

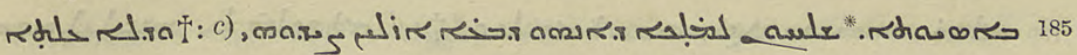

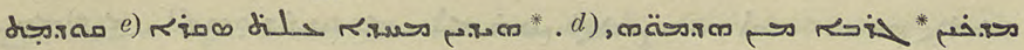

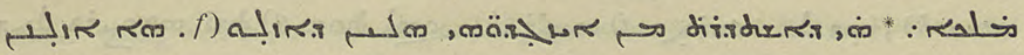

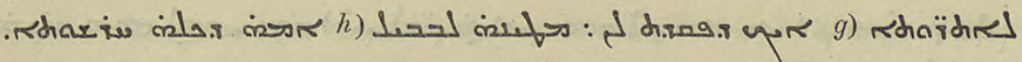

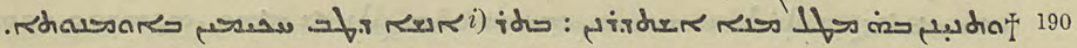

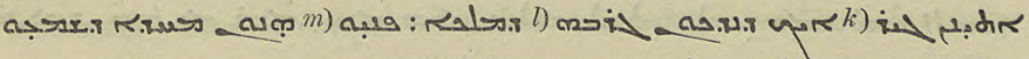
195

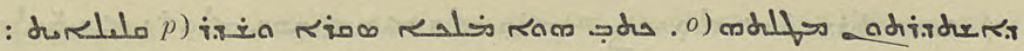

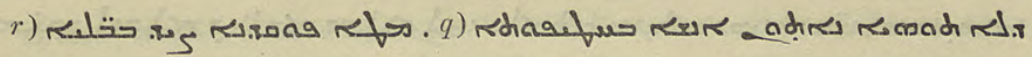

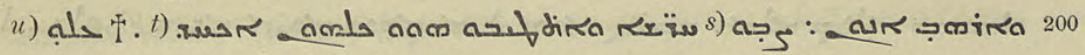

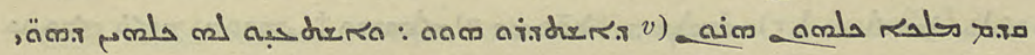

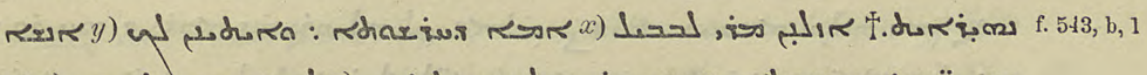

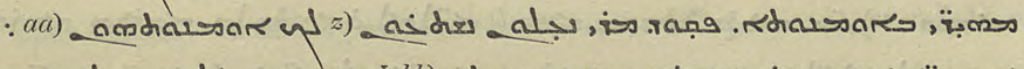

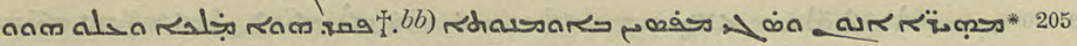

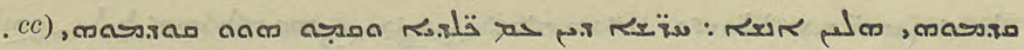

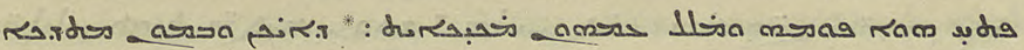

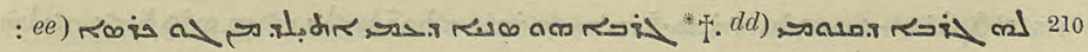

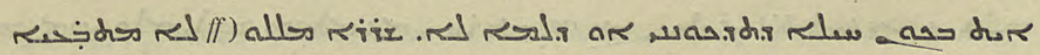

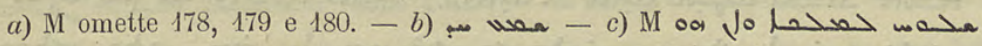
(a)

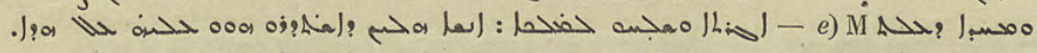

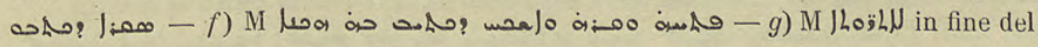

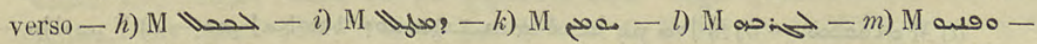

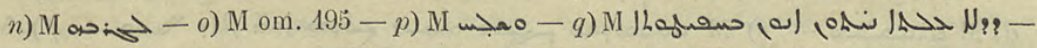

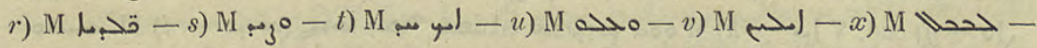
y) M 0 - - ) M (a) ( - 207 -

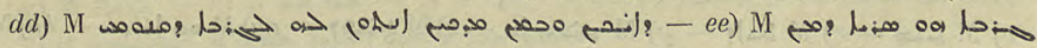

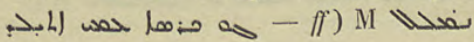




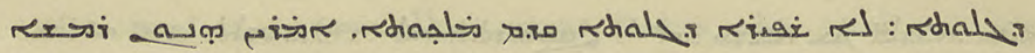

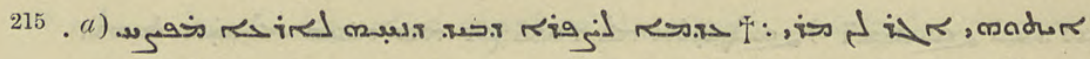

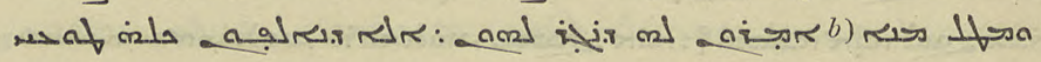

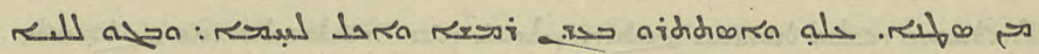

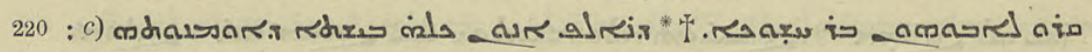

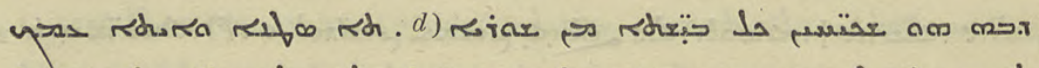

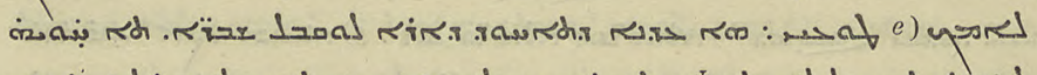

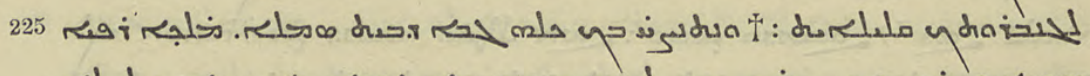

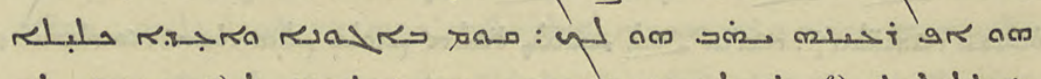

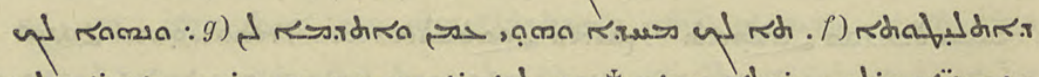

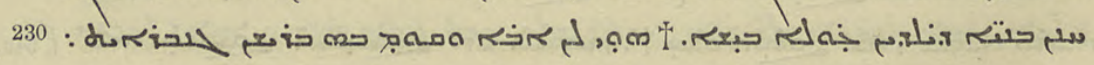

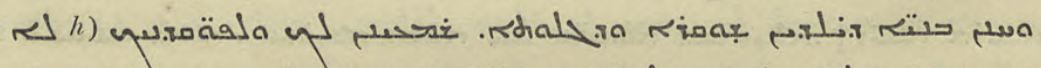

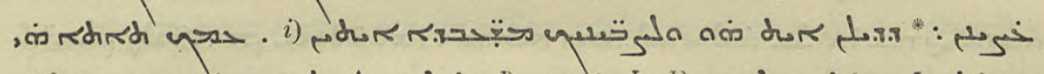

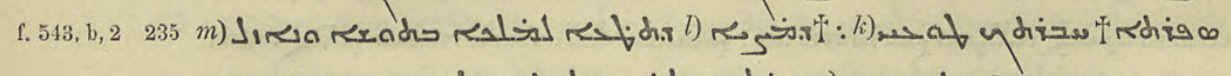

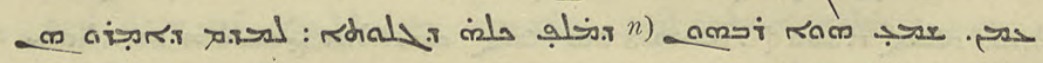

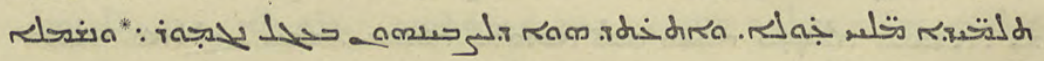

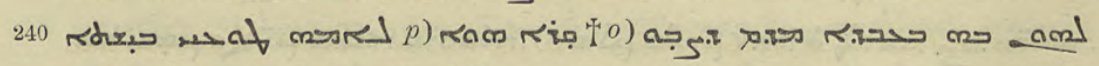

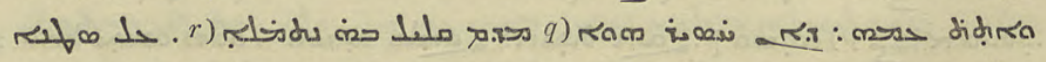

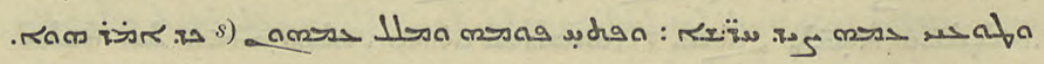

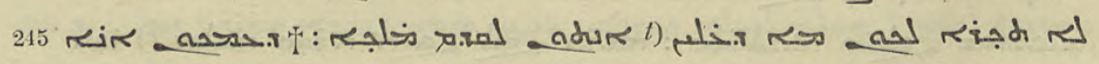
acia

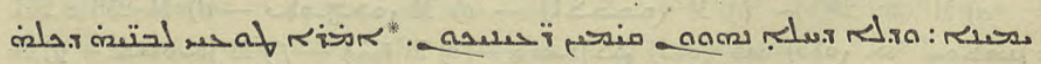

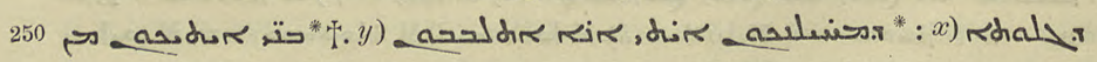

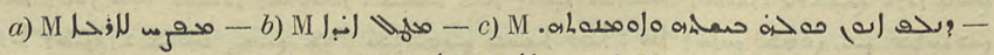

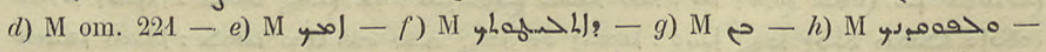

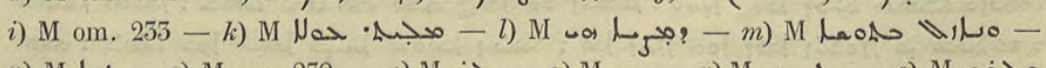
n) M b; - o) M om. $239-p$ ) M (م) - $t$ ) M

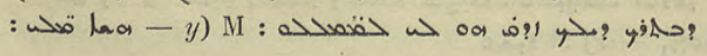




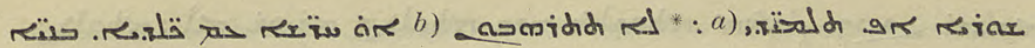

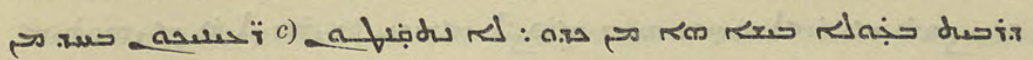

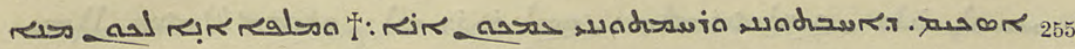

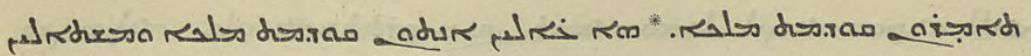

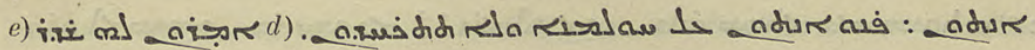
260

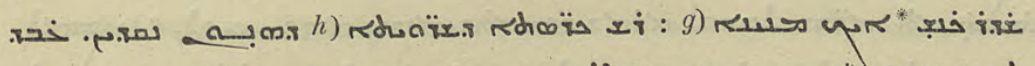

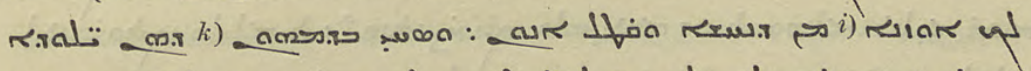

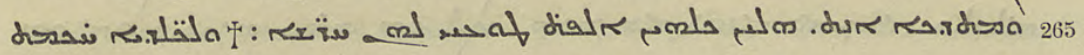

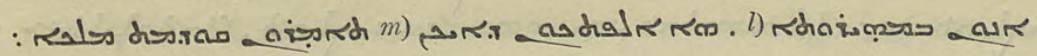

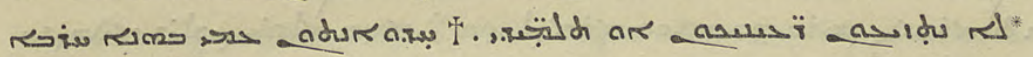

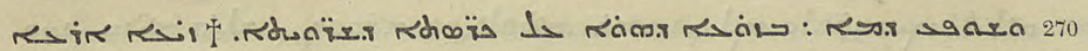

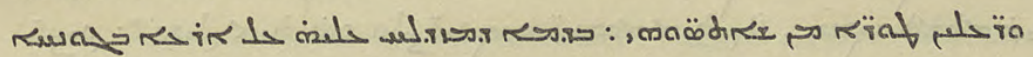

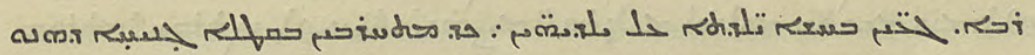

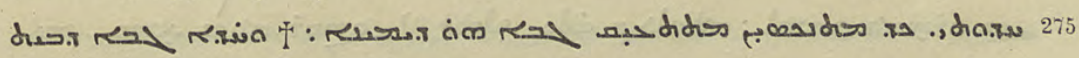

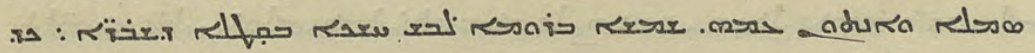

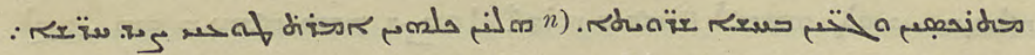

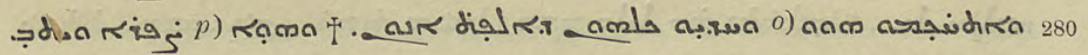

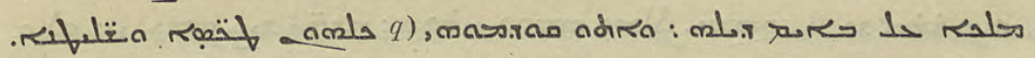

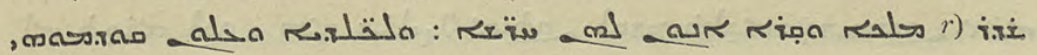

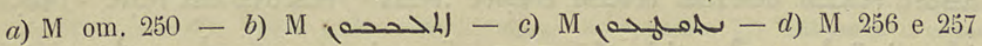

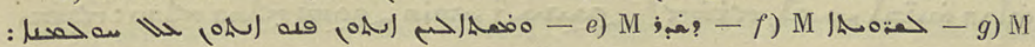

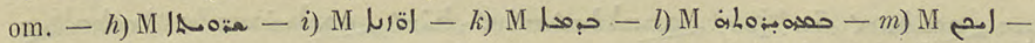

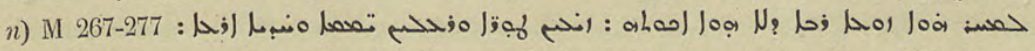

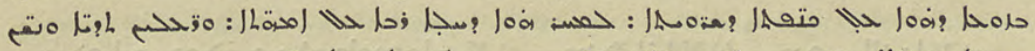

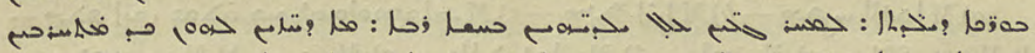

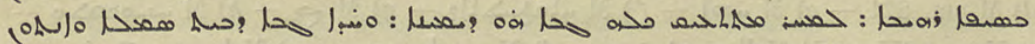

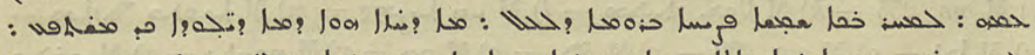

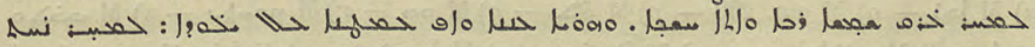
-

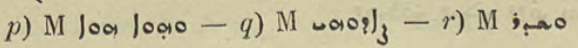




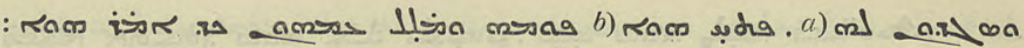

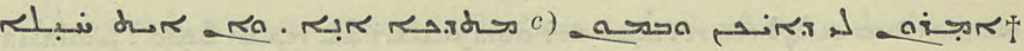

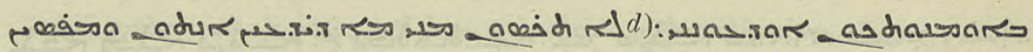

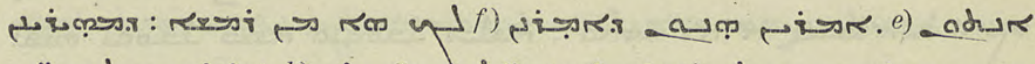

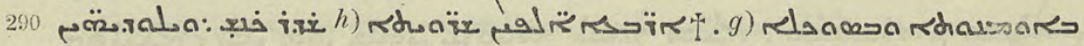

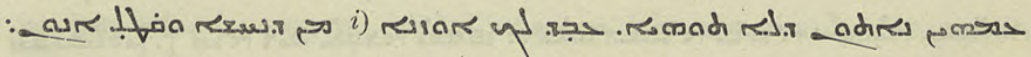

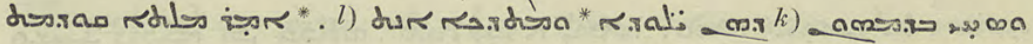

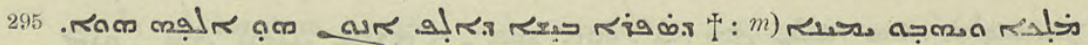

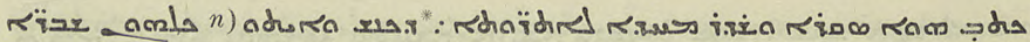

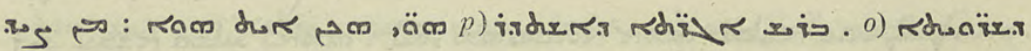

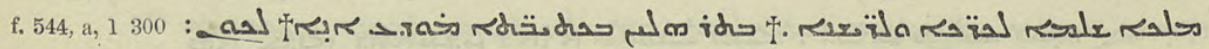

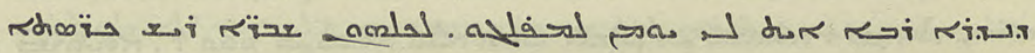

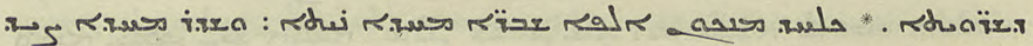
$305: q$ (q)

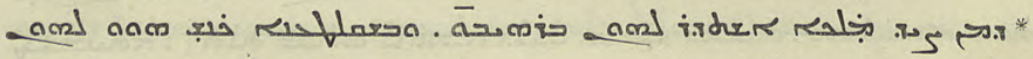

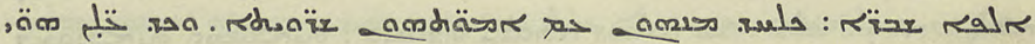

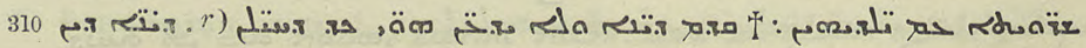

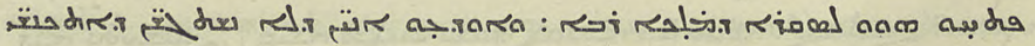

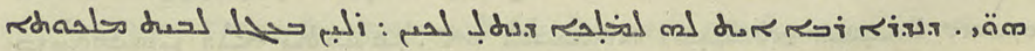

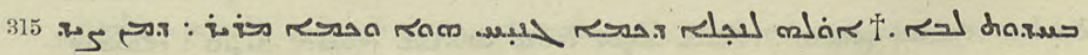

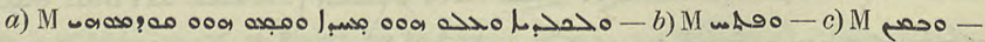

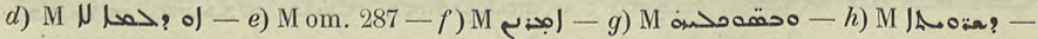

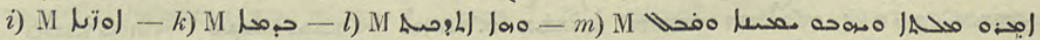

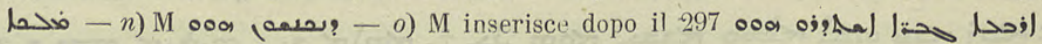

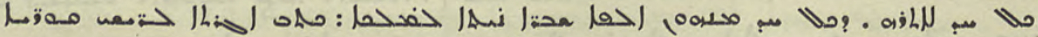

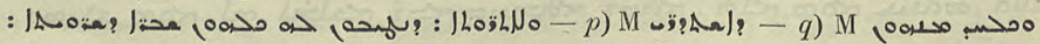

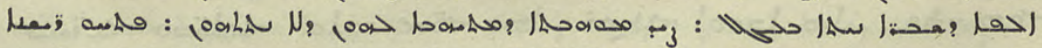

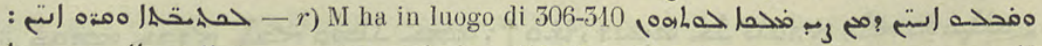

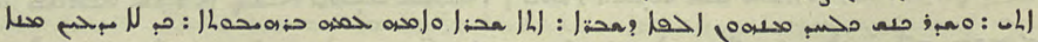
: فم quil termina il fol. 125, b, del cod. M, dopo che mancano uno o più fogli. 


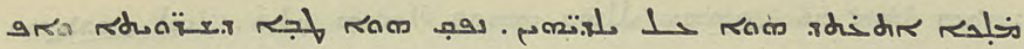

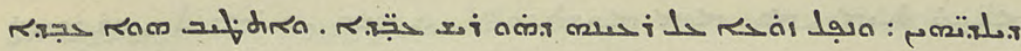
小市.

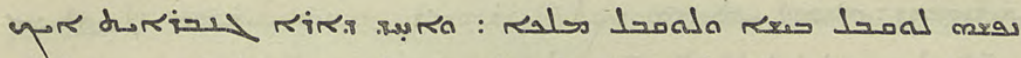

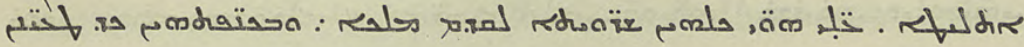

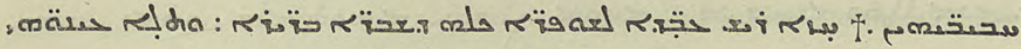

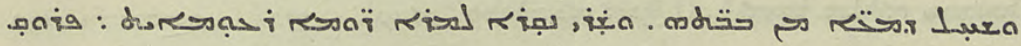

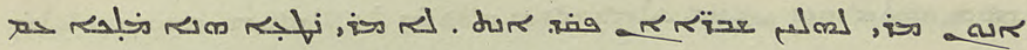

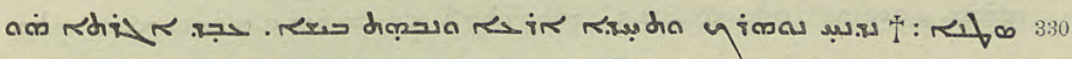

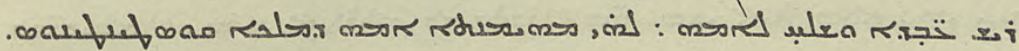

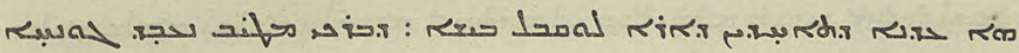
335

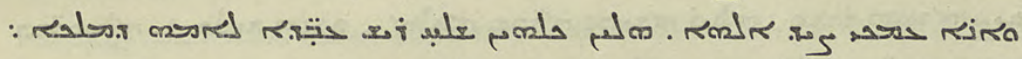

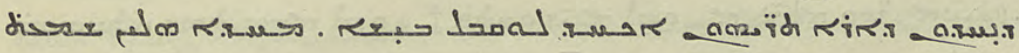

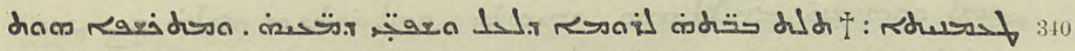

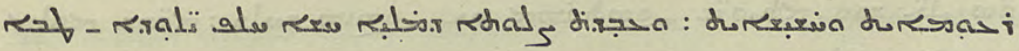

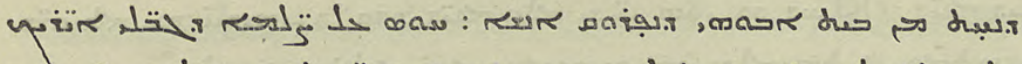

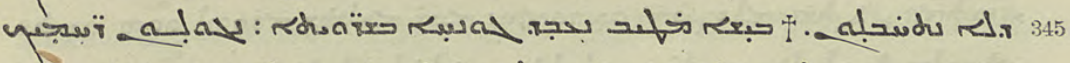

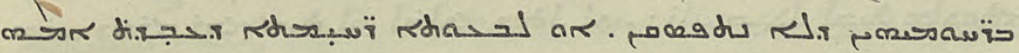

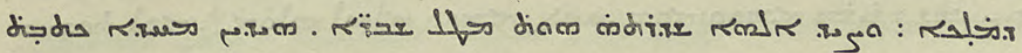

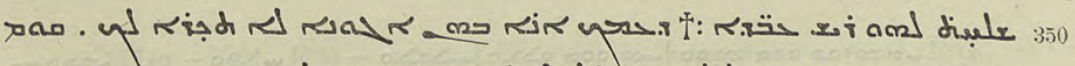

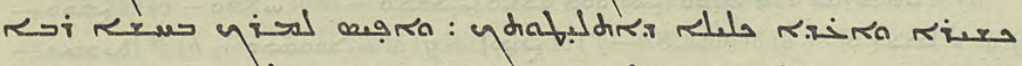

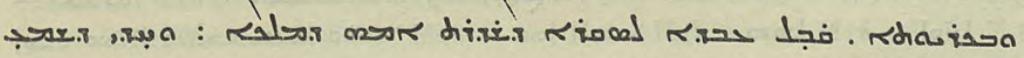
: 355

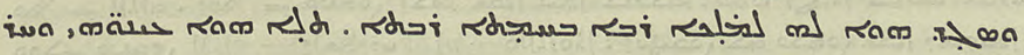

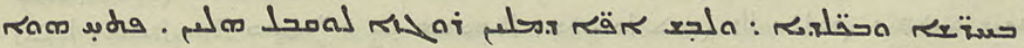
360 له s

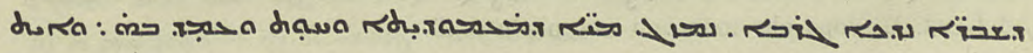




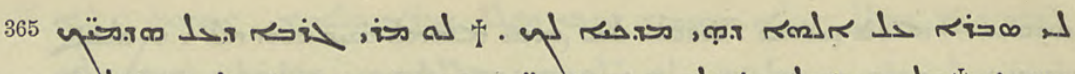

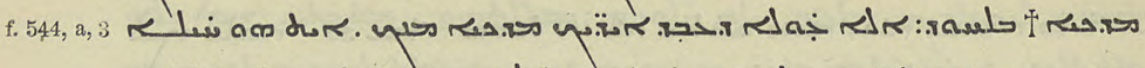

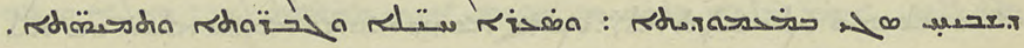

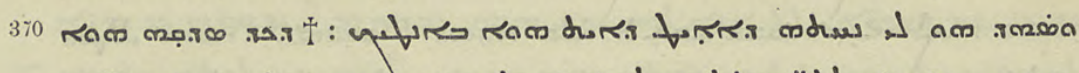

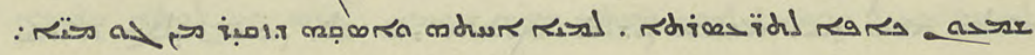

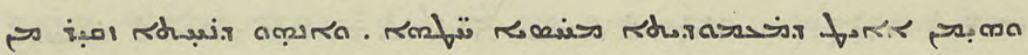

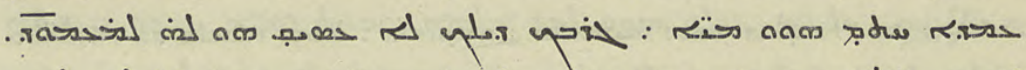

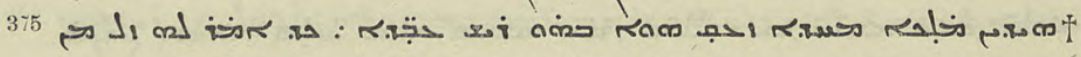

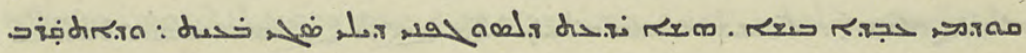

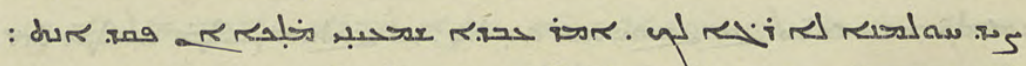

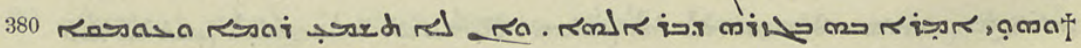

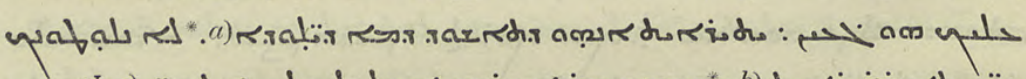

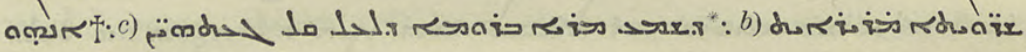

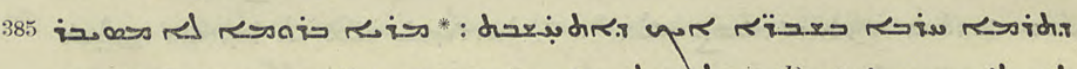

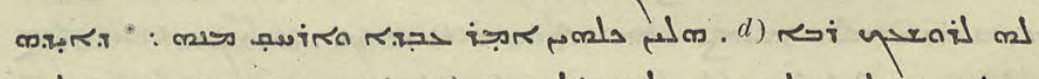

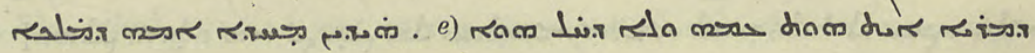

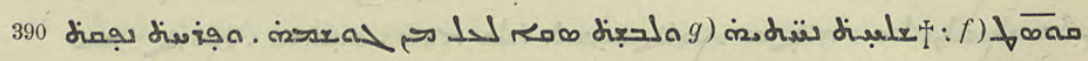

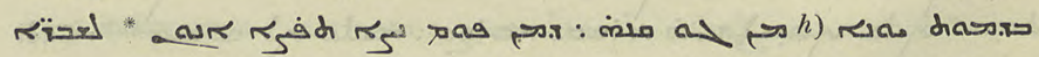

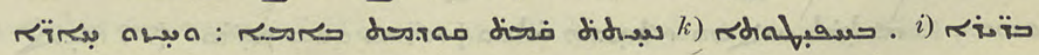

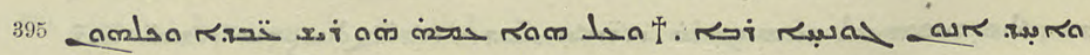

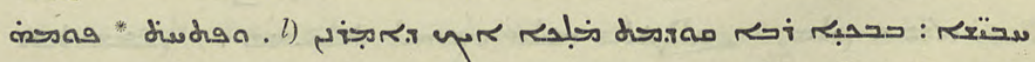

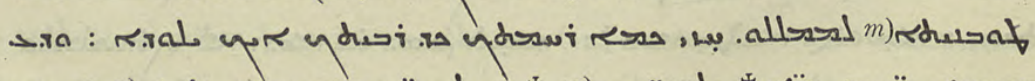

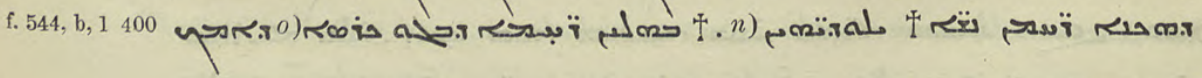

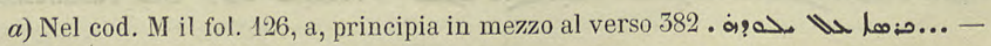

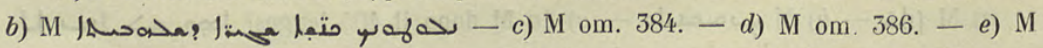
-

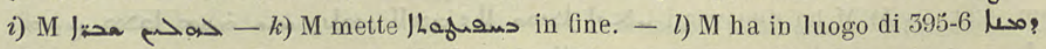

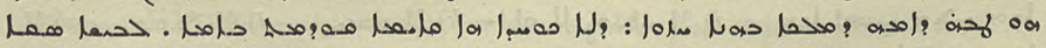

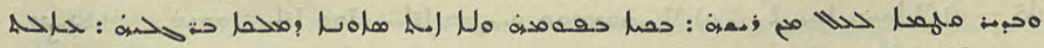
صis of 
مص

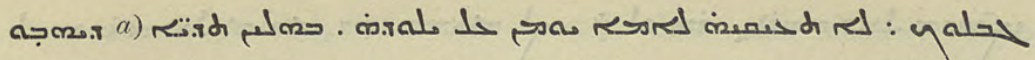
.

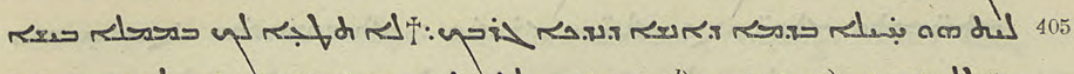

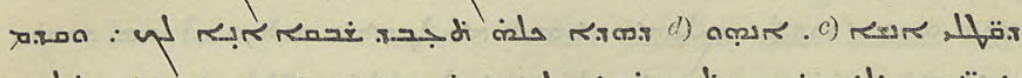

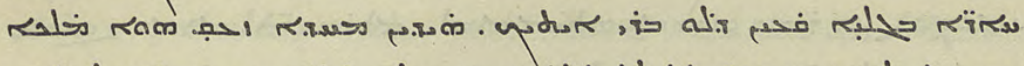

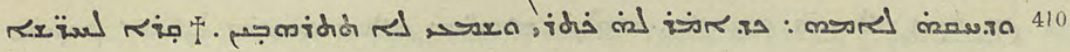

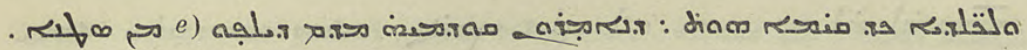

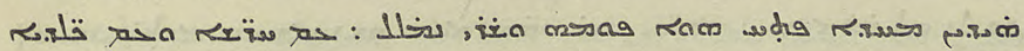

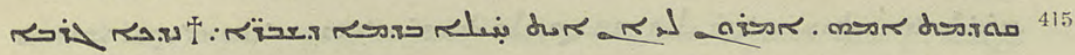
א'

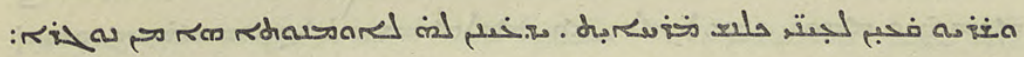

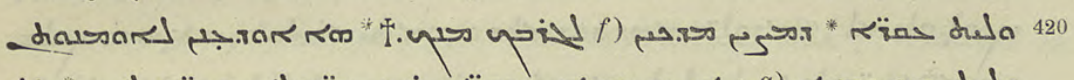

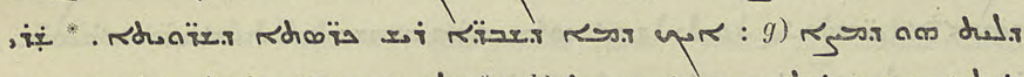

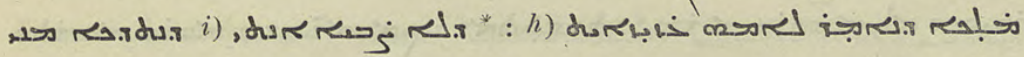

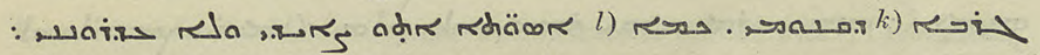

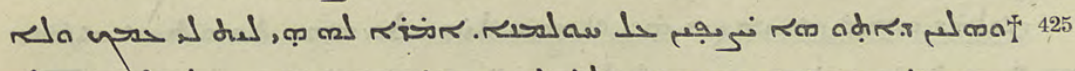

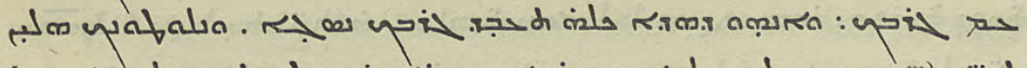

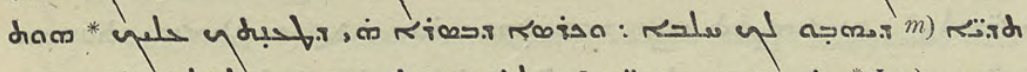

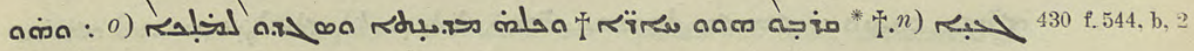

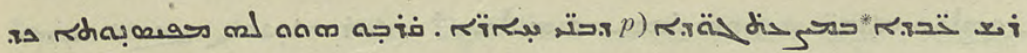

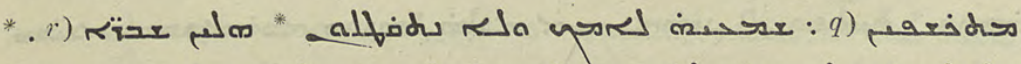
435

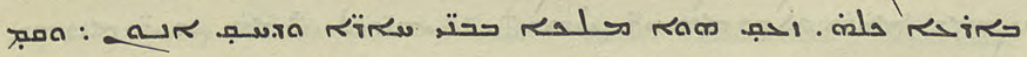
كل

a) M (N

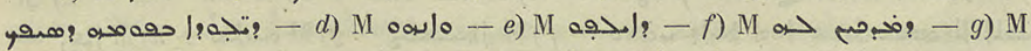
om. 420. - h) M ha due versi u

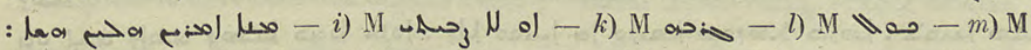

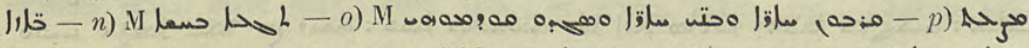

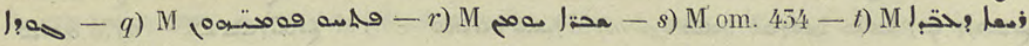




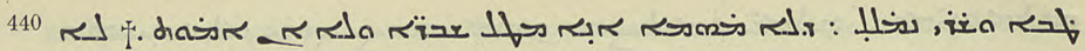

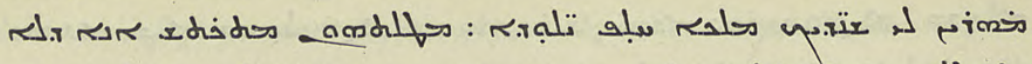

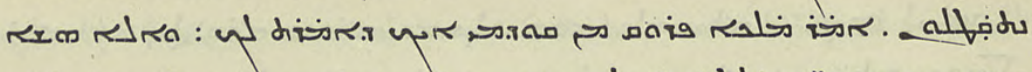

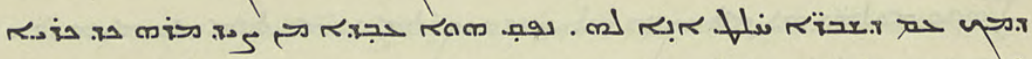

445 a

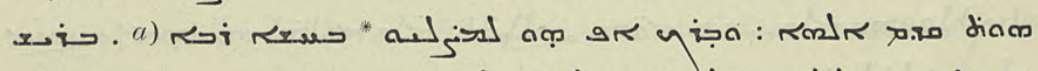

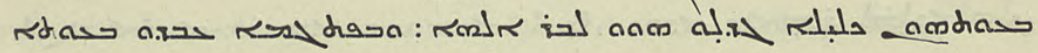
450 q

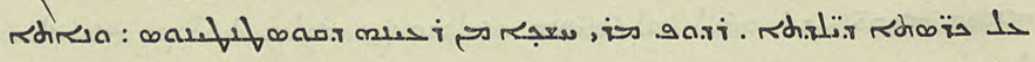

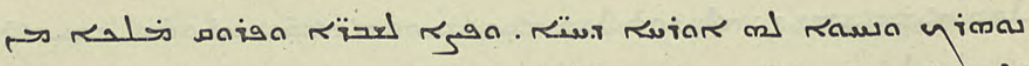

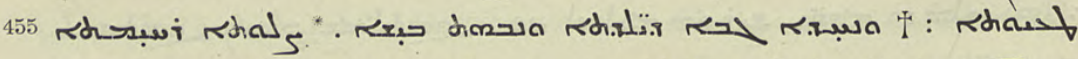

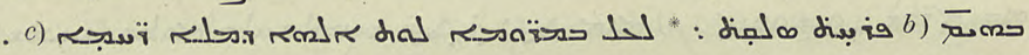

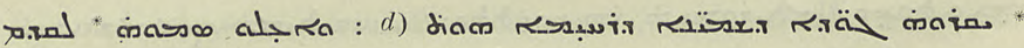

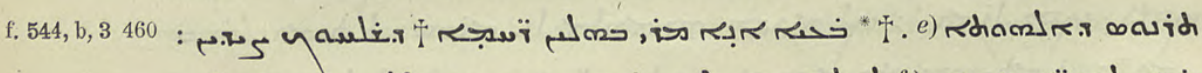

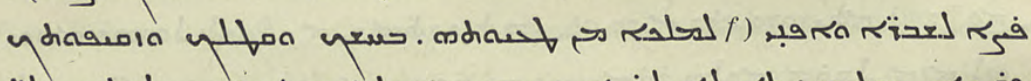

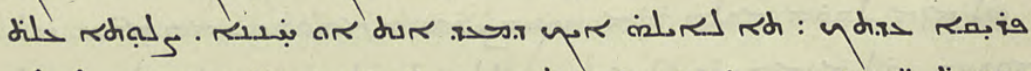

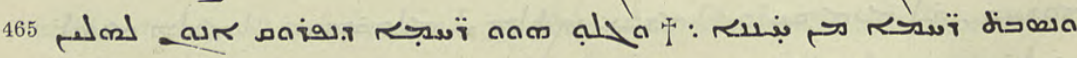

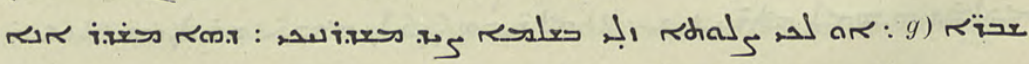

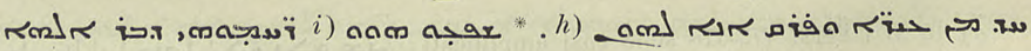

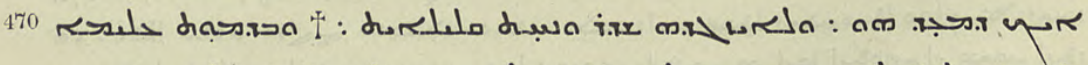

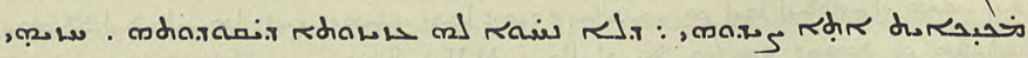

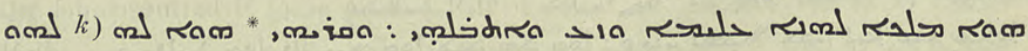

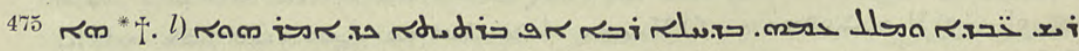

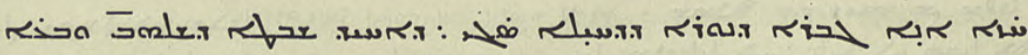

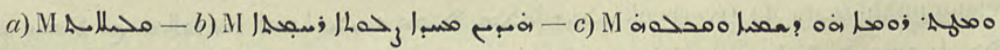

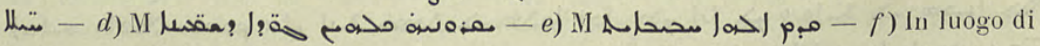

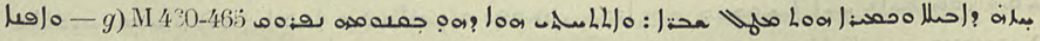

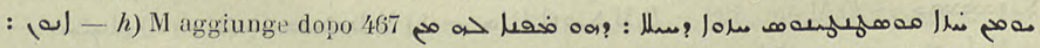

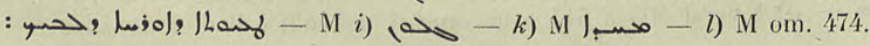




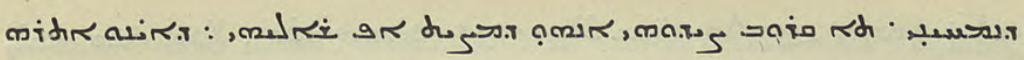
م

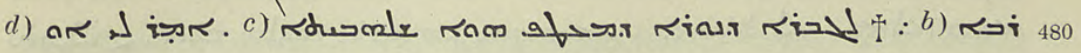

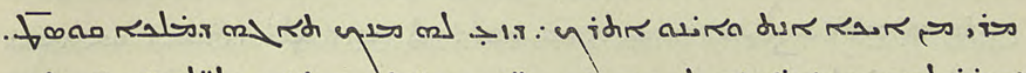

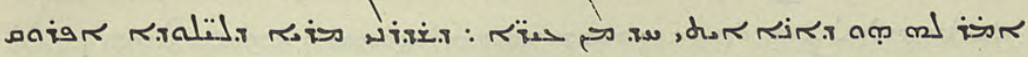

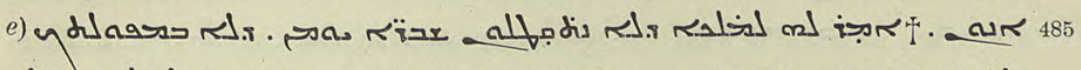

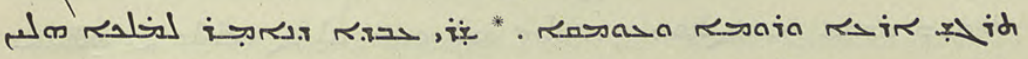
an ram ل

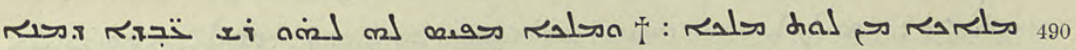

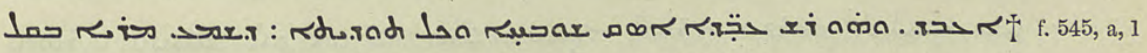
S 495 ת

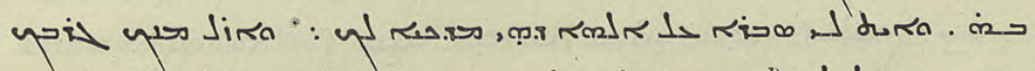
Rم:

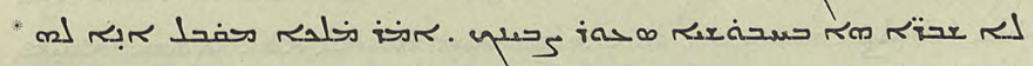

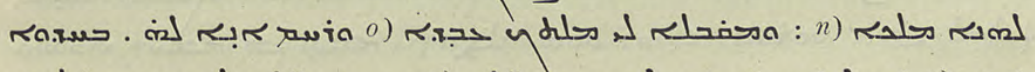
i

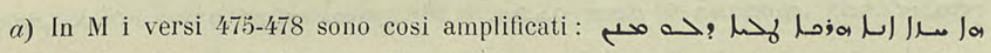

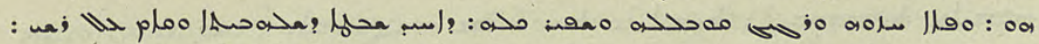
osal:

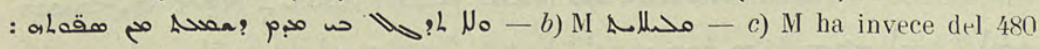

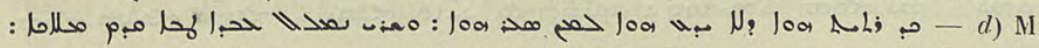

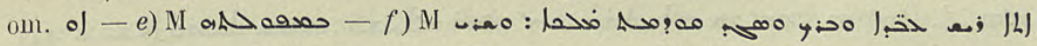
:

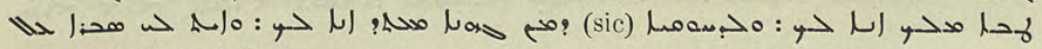

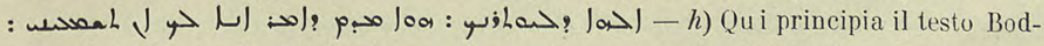

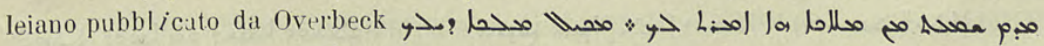

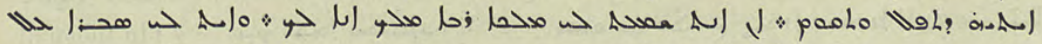

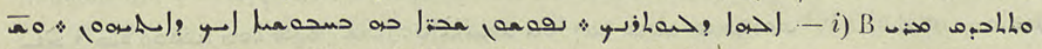

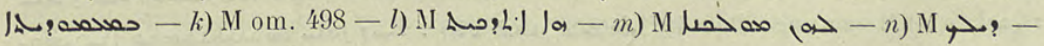
o) M I مسب - p) I versi 503 a 545 sono omessi nel codice Bodleiano. 


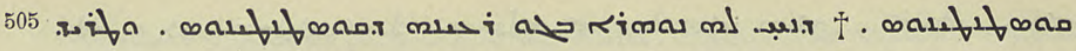
ف y)

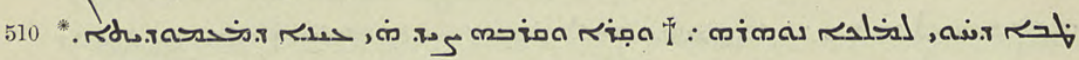

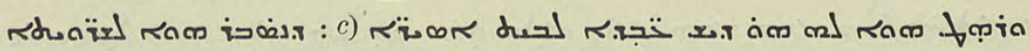

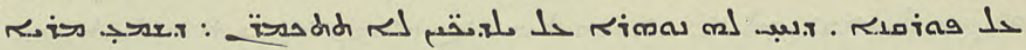

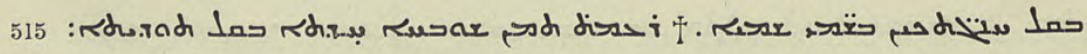

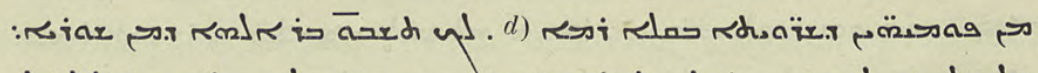

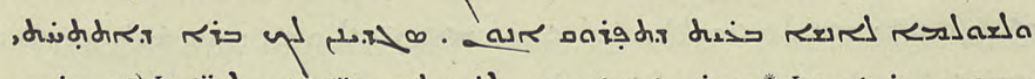

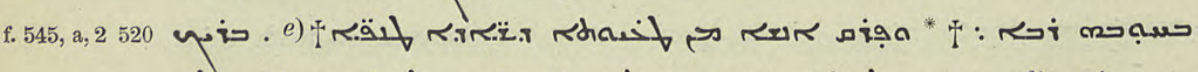

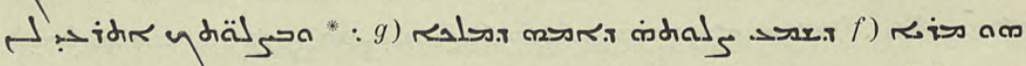
a مa

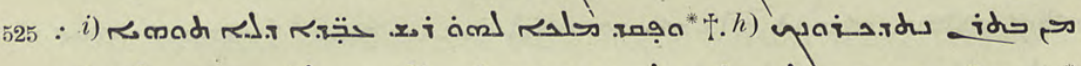
"

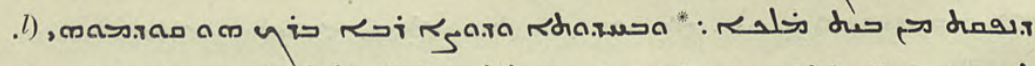

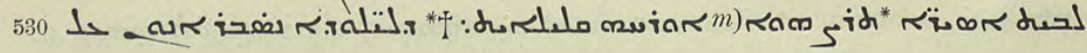

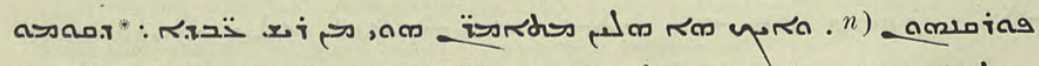

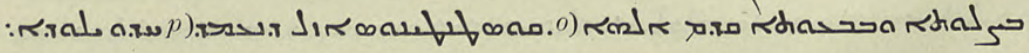

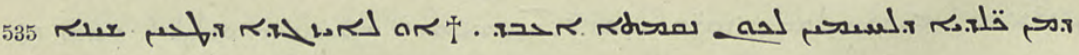

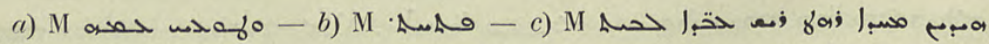

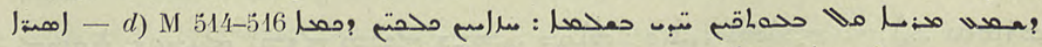

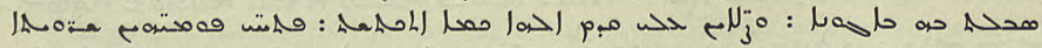

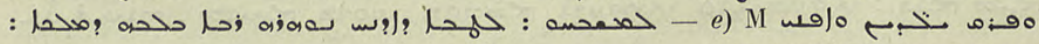

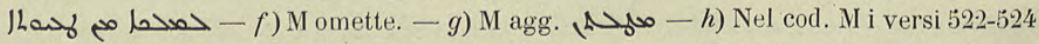

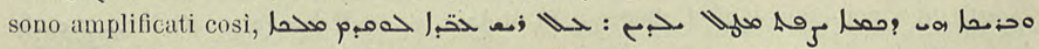

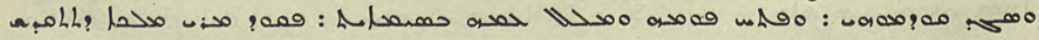
: :

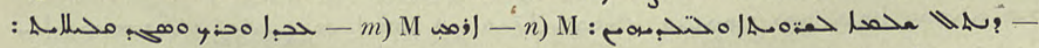

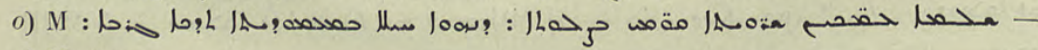
p) II Uת 


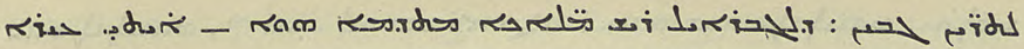
or 540

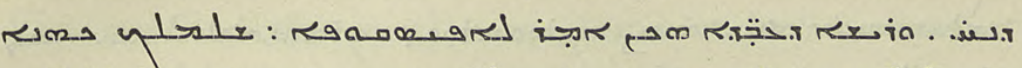

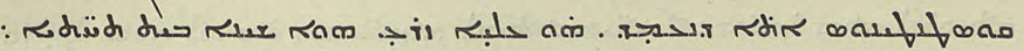

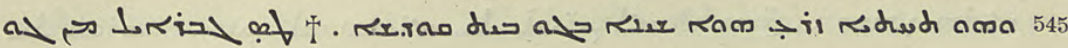

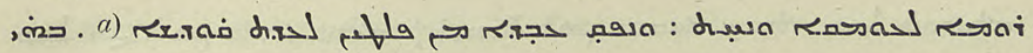

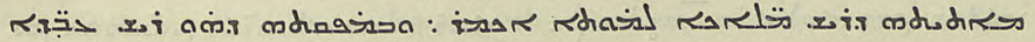

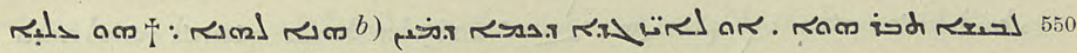

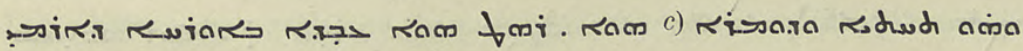
rom mal.fif

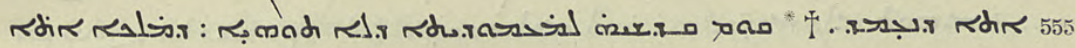

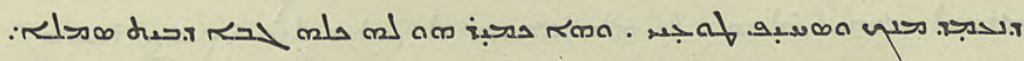
נה.بes . Kल 560

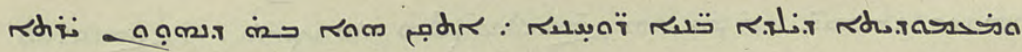

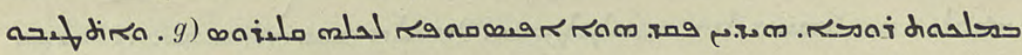
565

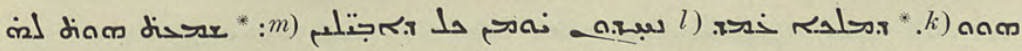

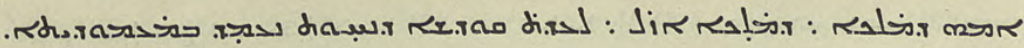

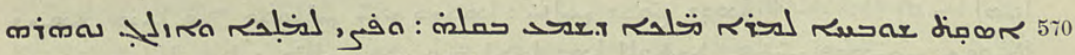

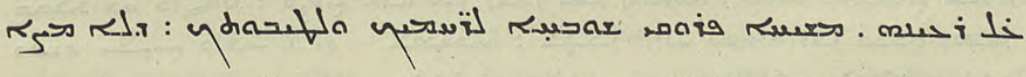

a) Qui riprincipia il Cod. Bod. - b) M lo حبر (

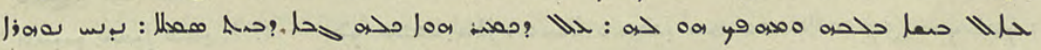

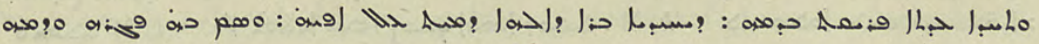

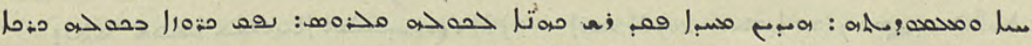

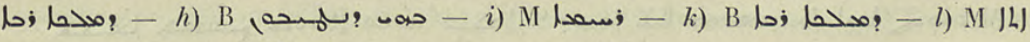

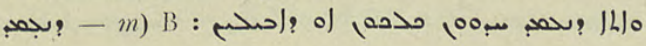




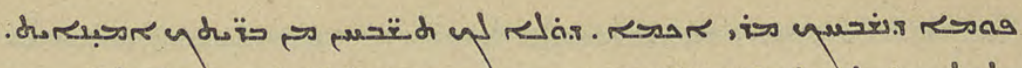

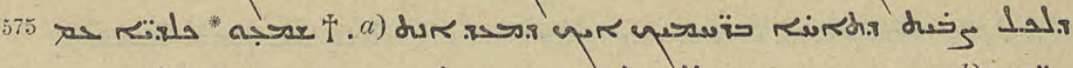

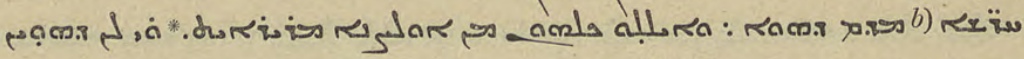

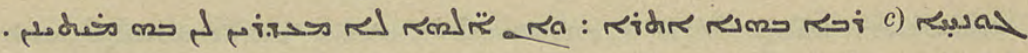
580 id * ה

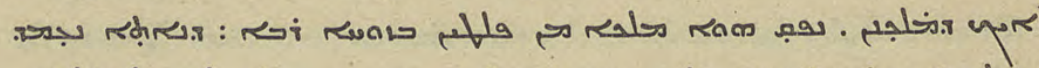

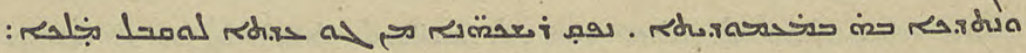

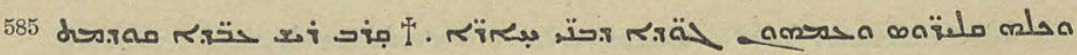

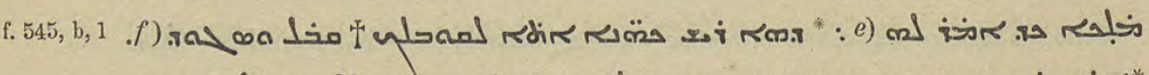

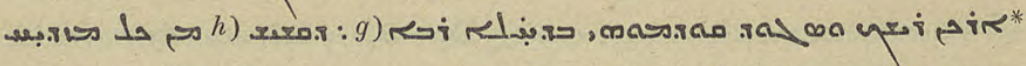

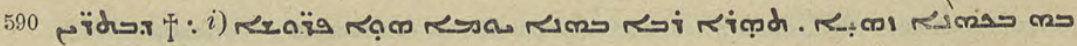

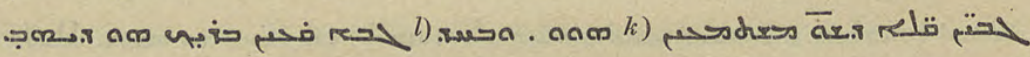

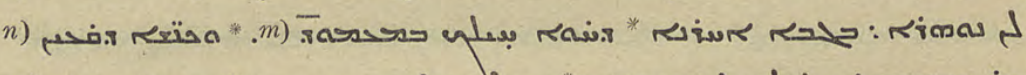

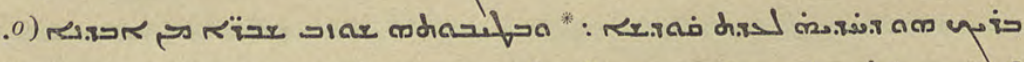

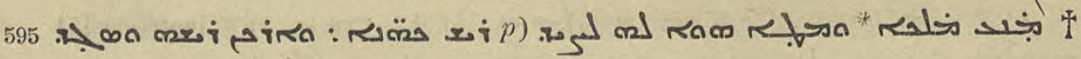

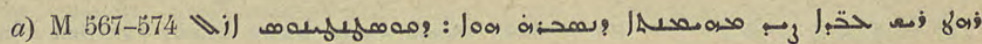

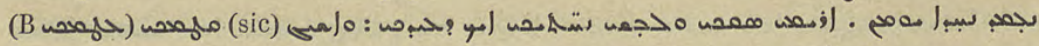

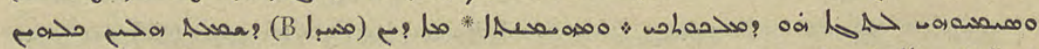

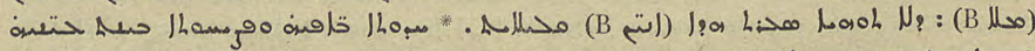

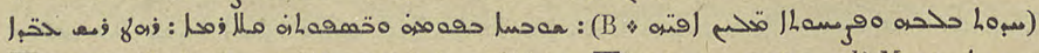

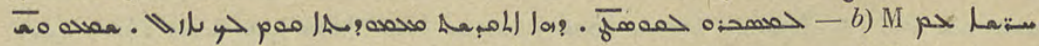

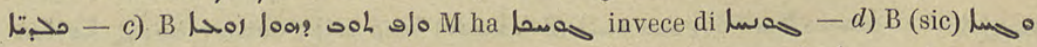

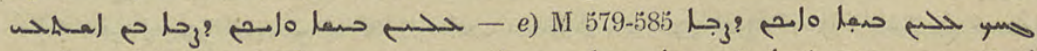

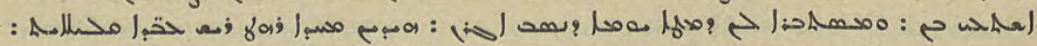

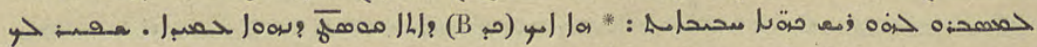
: (Bnoltre seguono in B

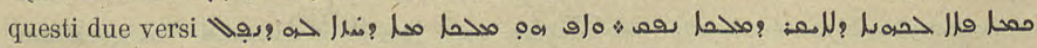

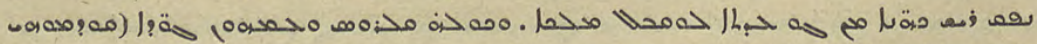

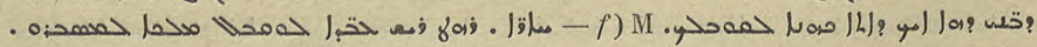

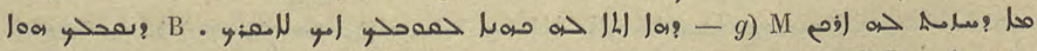

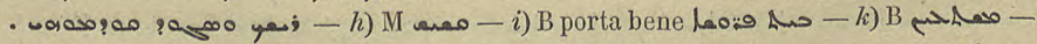

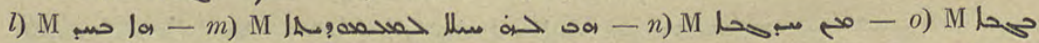

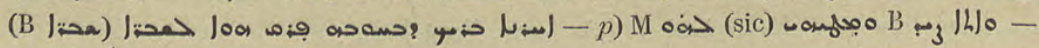




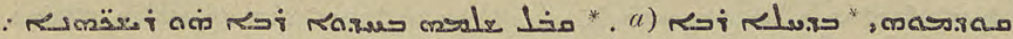

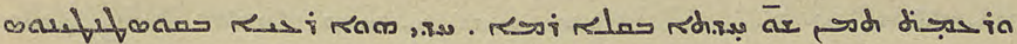

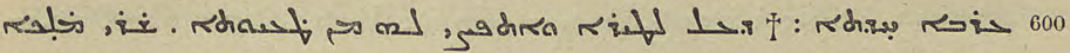

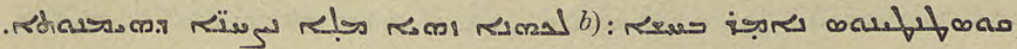

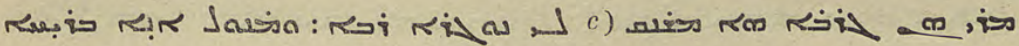

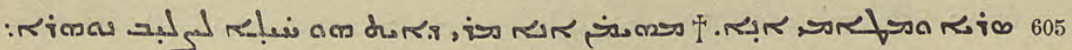

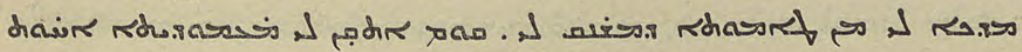

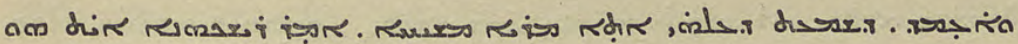

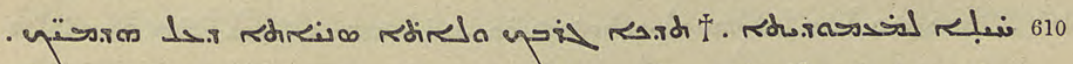

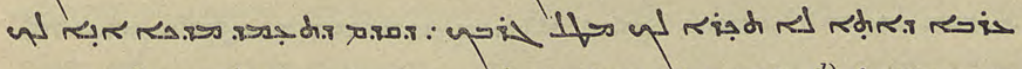

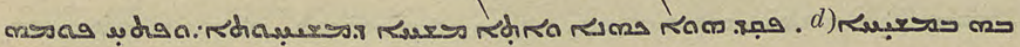

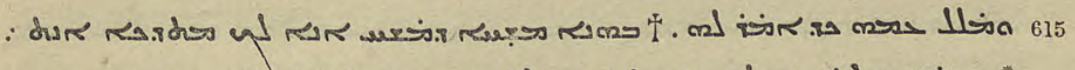

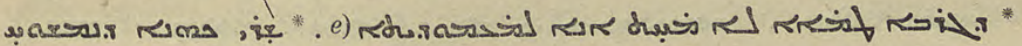

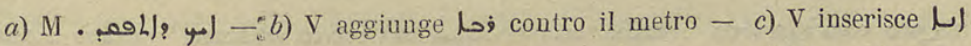

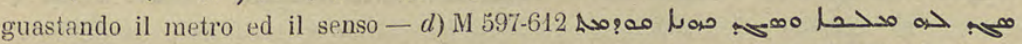

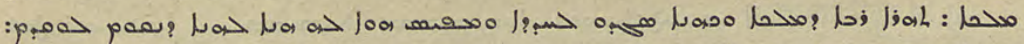

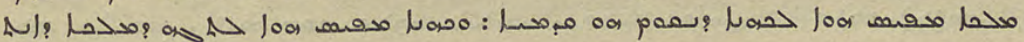

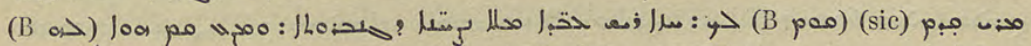

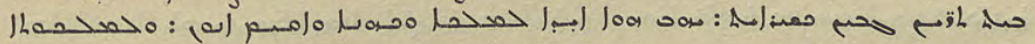

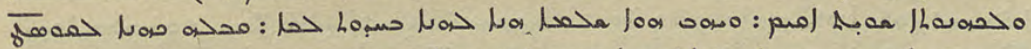

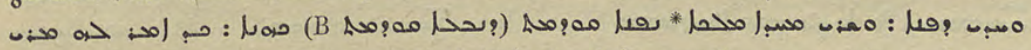

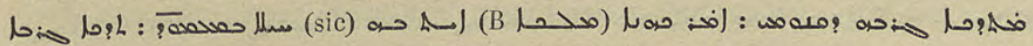

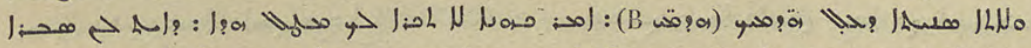
: B مب poscia segue in B un tratto che non trovasi altrove e che citiamo testualmente da Overbeck: esso precede il verso $613 \mathrm{~V}$. (00m :

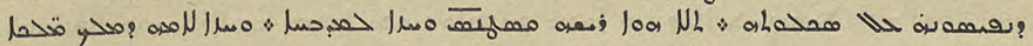

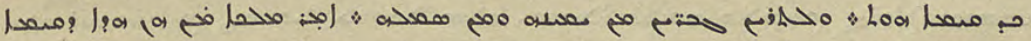

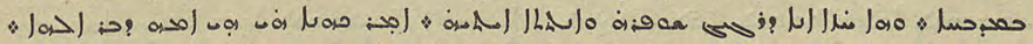
?

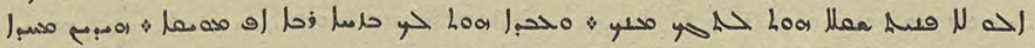

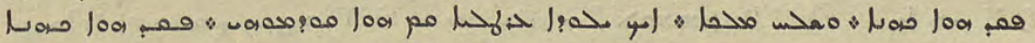

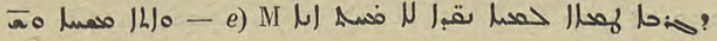




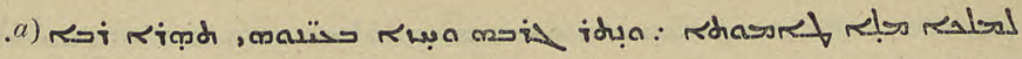

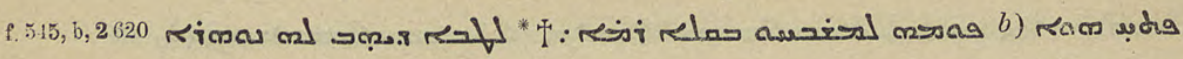

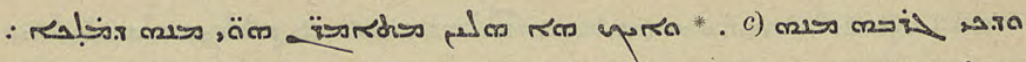

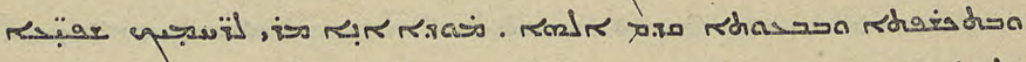
625 rẹ r.

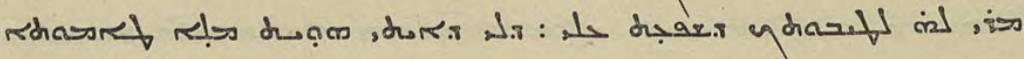

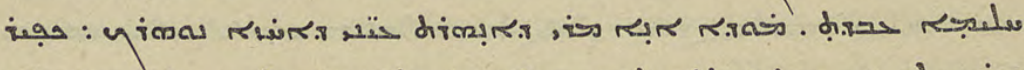

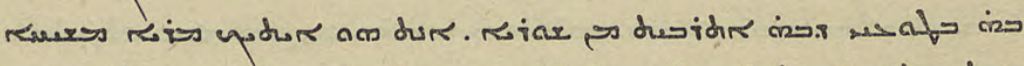

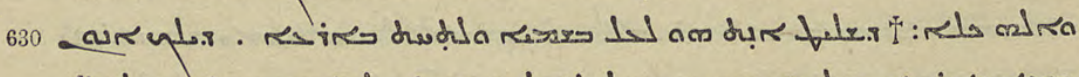

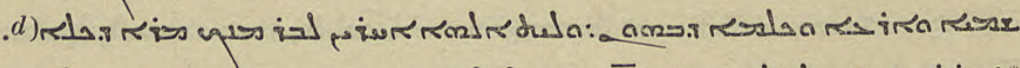

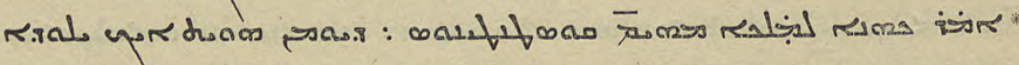
$635 \mathrm{Kh}:$ : q id a

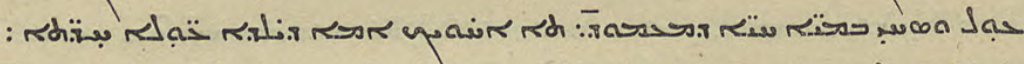

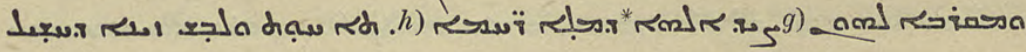
640 Ja da

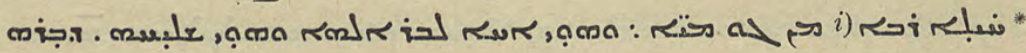

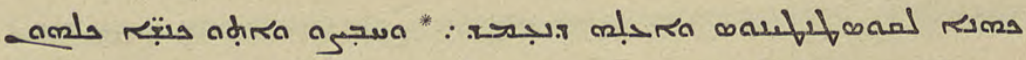

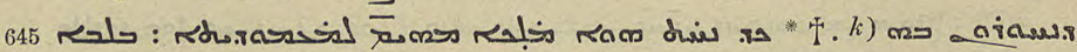

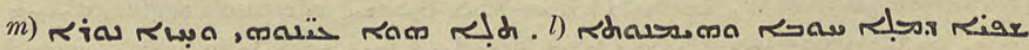

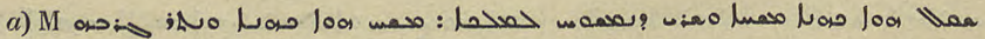
o

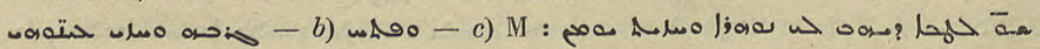

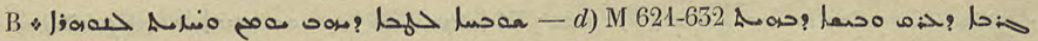

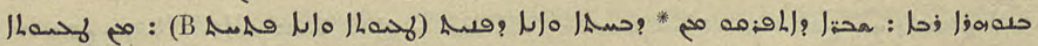

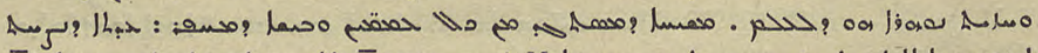

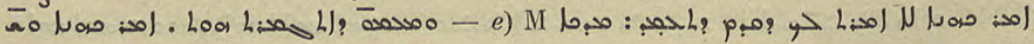

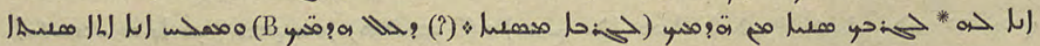

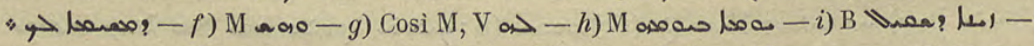

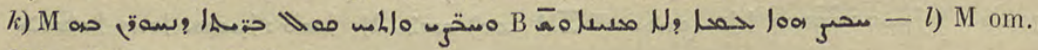
645 e $646,-m$ ) M liod 


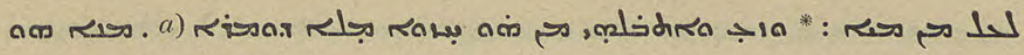

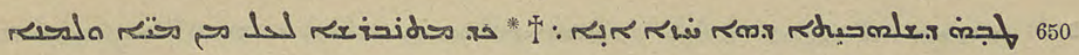

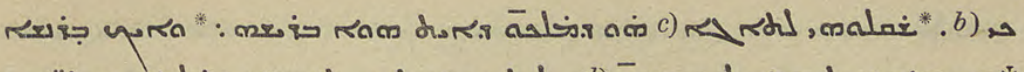

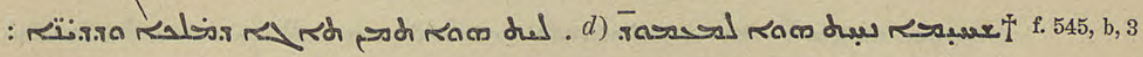

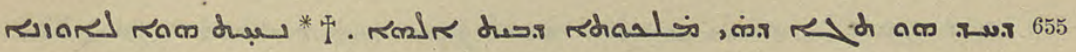

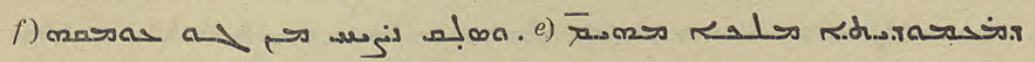

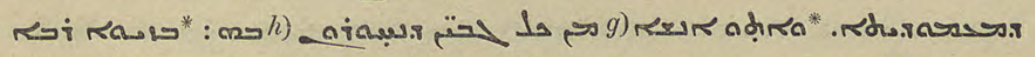

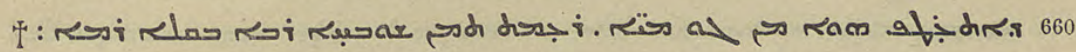

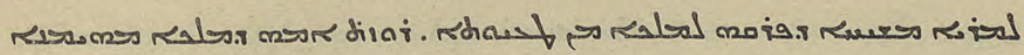

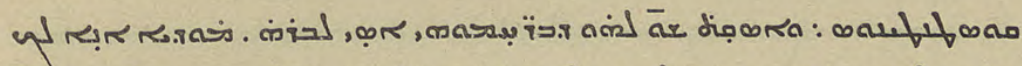

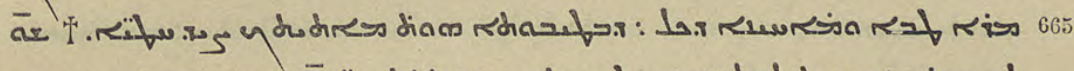

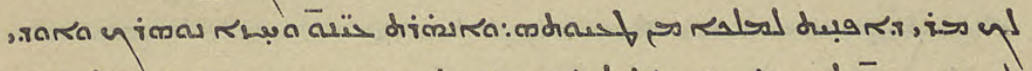

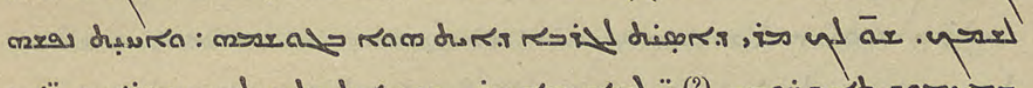

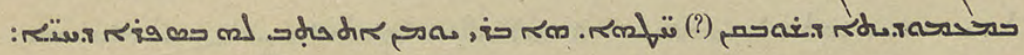

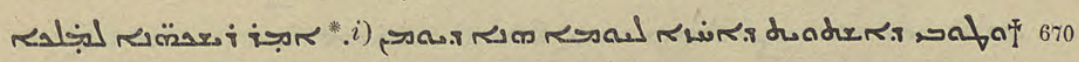

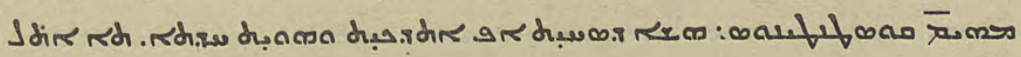

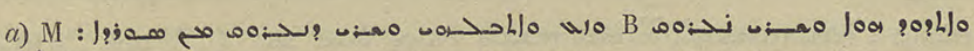

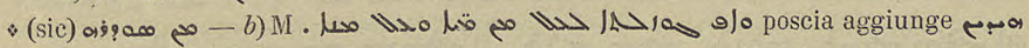

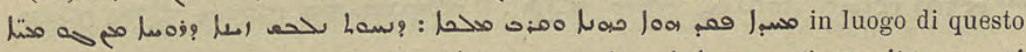
verso e del seguente leggesi in B * ol

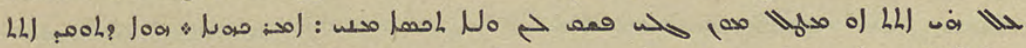

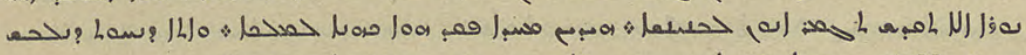

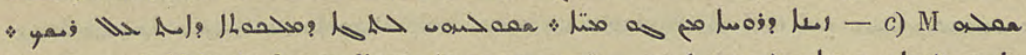

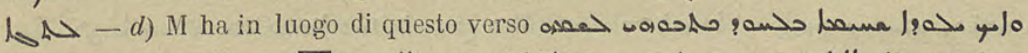

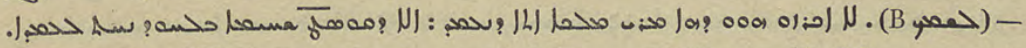

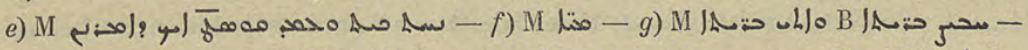

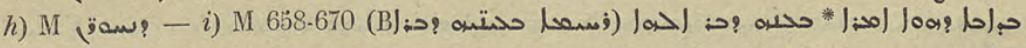

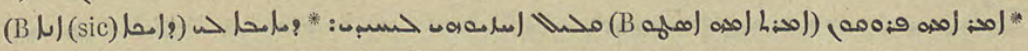

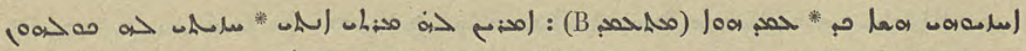

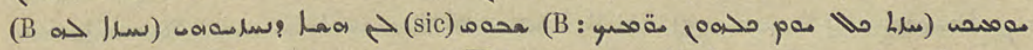

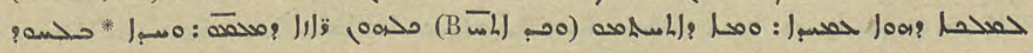

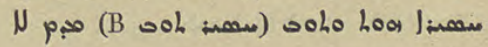




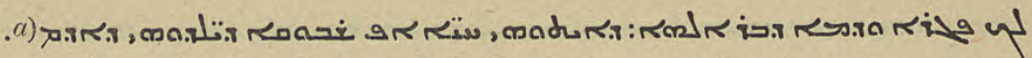
675 Ri

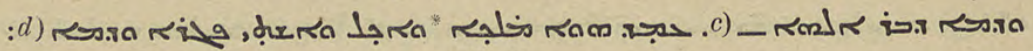

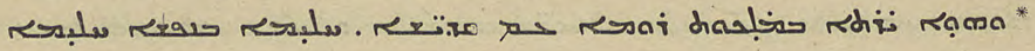

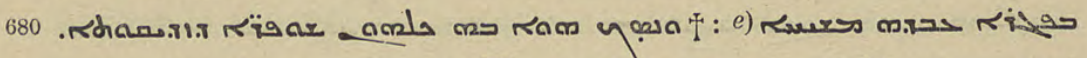

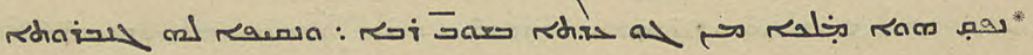

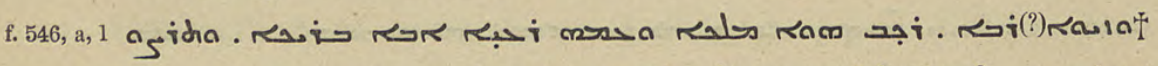

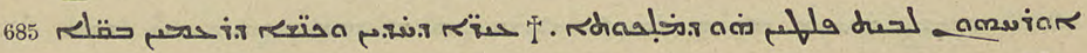

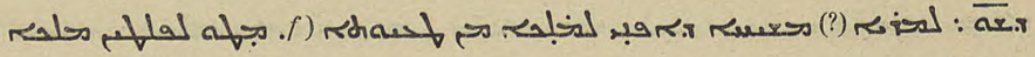

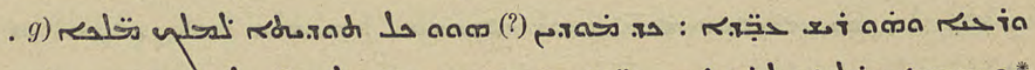

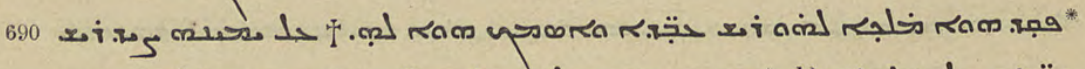

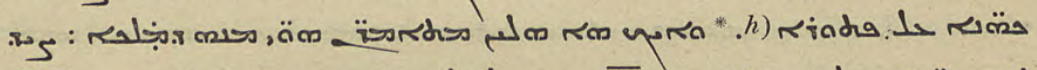

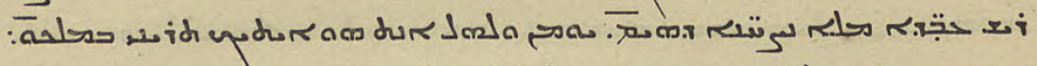

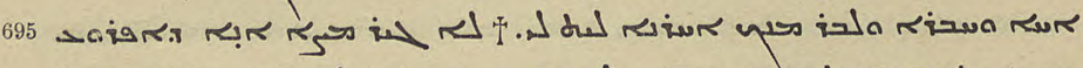

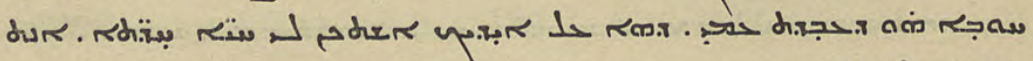
"1

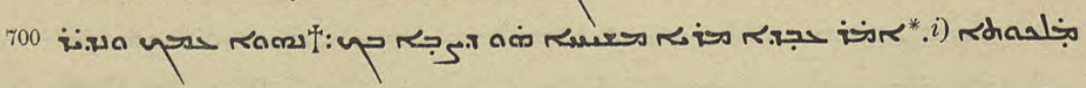

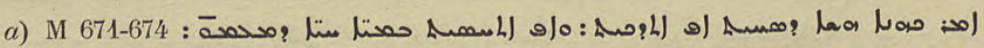

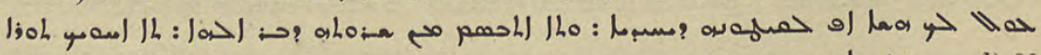

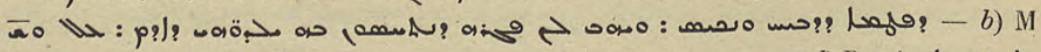

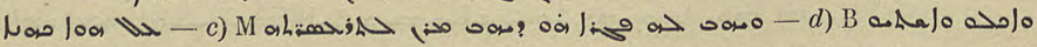

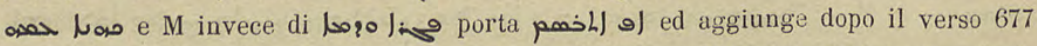

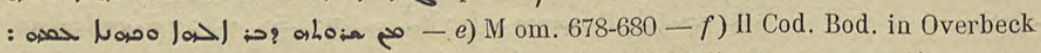
termina col verso 686. - g) M ha in luogo di 681-688 / Tha خ

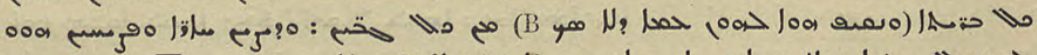

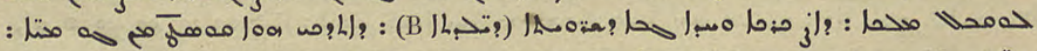

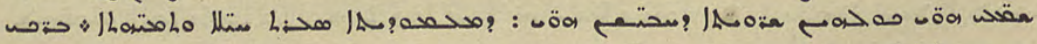

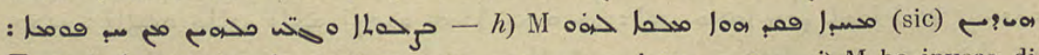

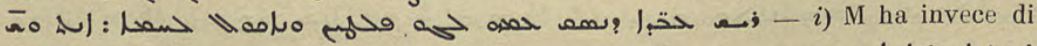

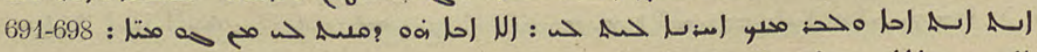
: wat pallo 


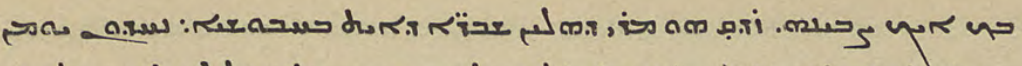

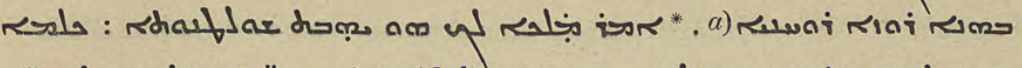

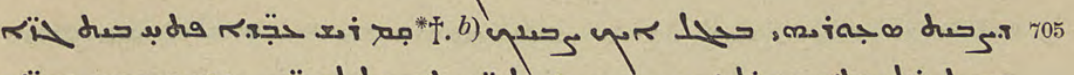

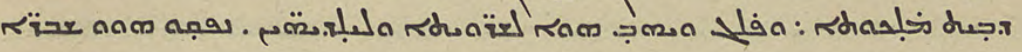

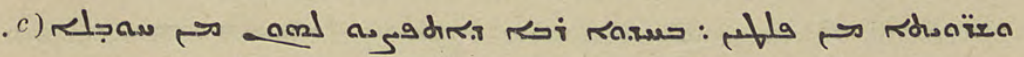
710

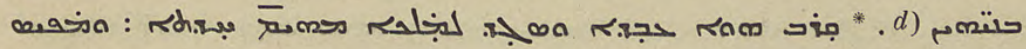

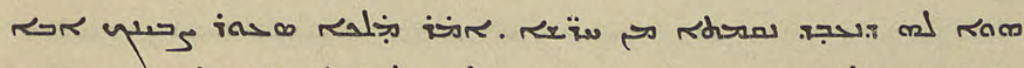

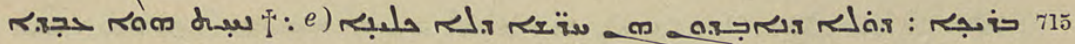

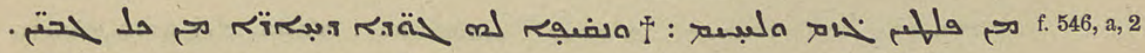
ص.

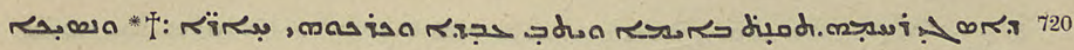

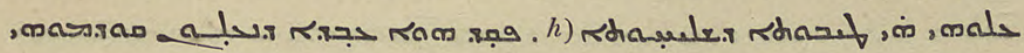

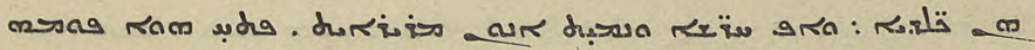

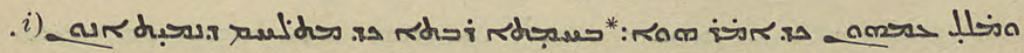

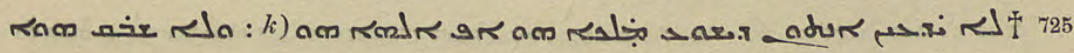

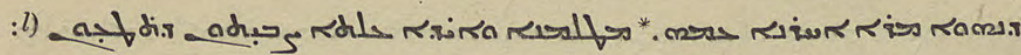

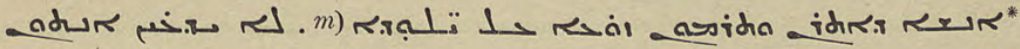
730

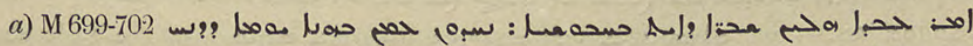

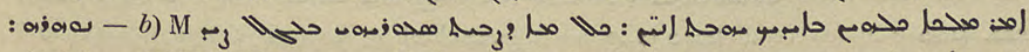

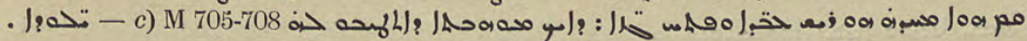

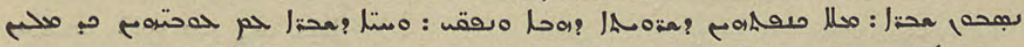
-

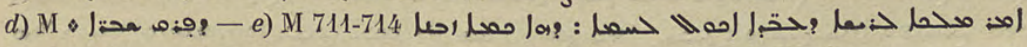

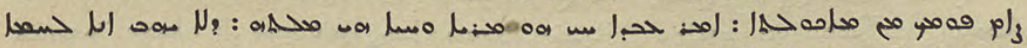

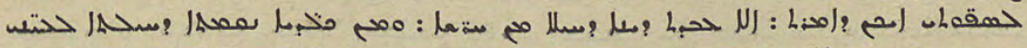

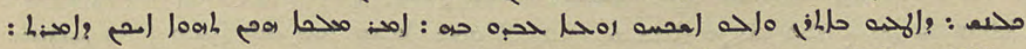

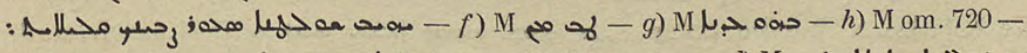

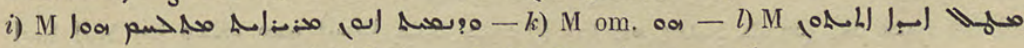

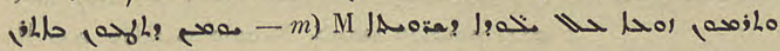


R.

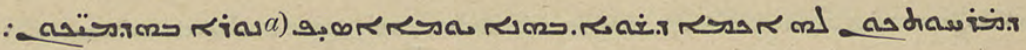

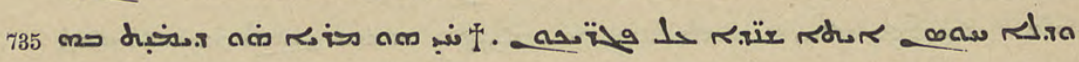

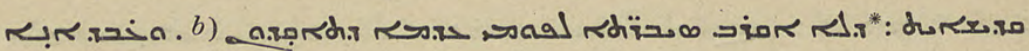

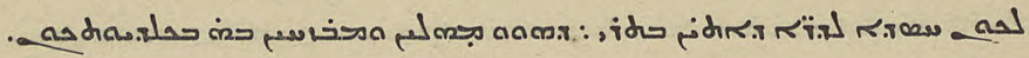

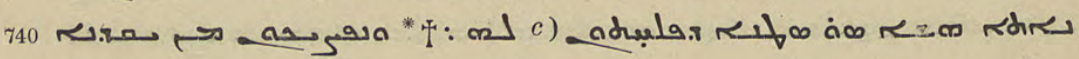

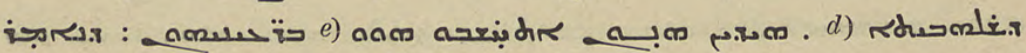

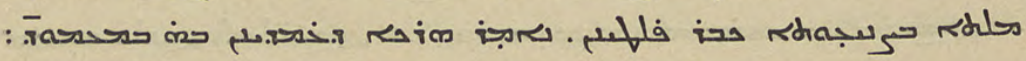
745 arsa

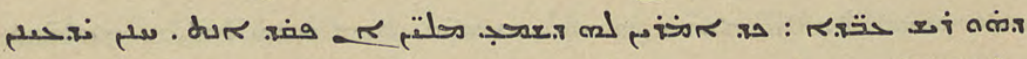
: am

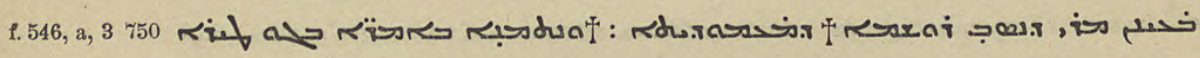

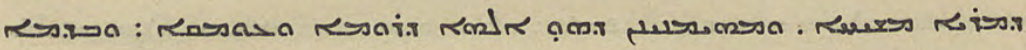

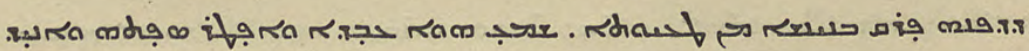
755 adus

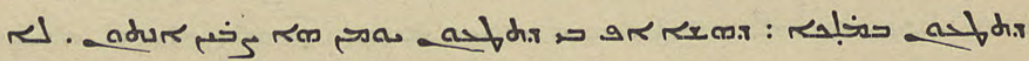

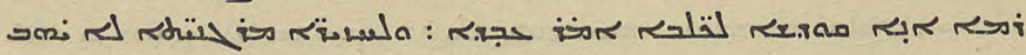

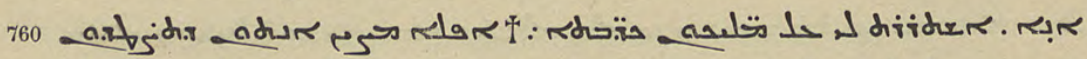

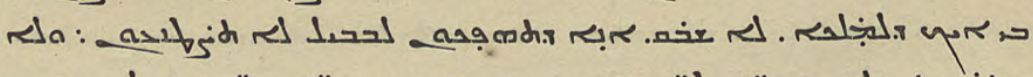

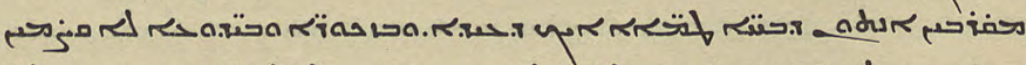

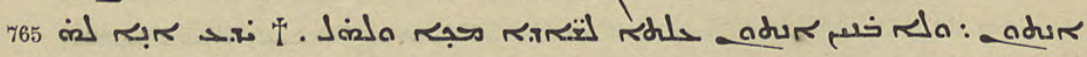

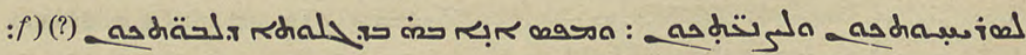

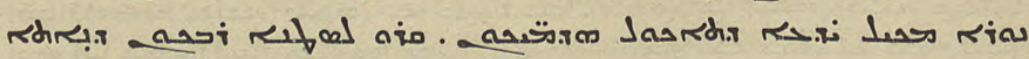

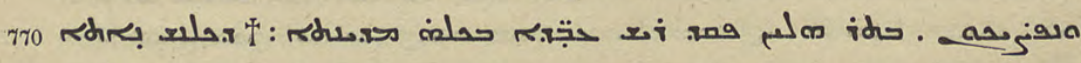

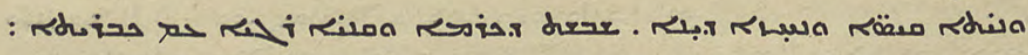

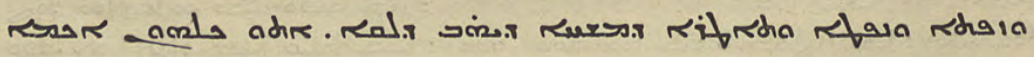

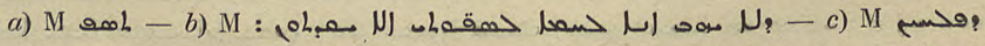

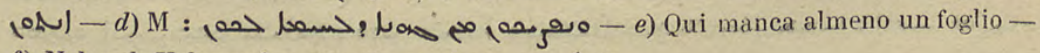
f) Nel cod. V leggesi a quanto sembra (anohol? 
?

775

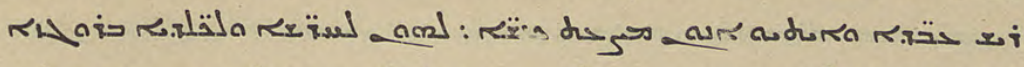

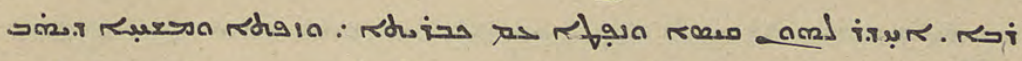
780

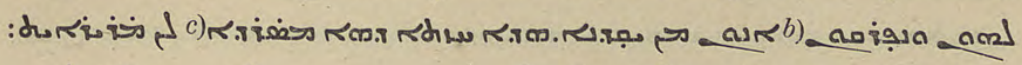

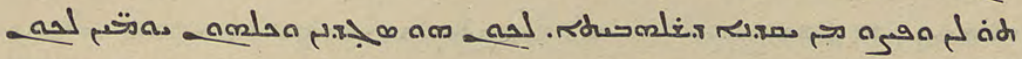

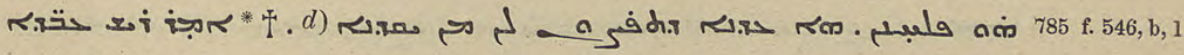

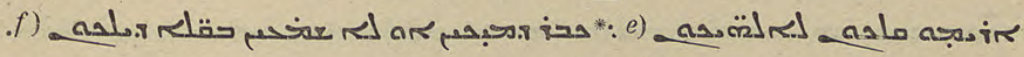

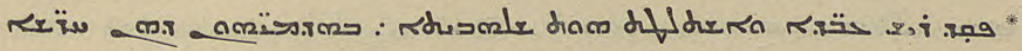
790

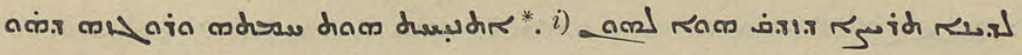

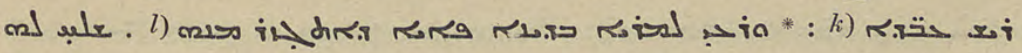
דبلar : 795

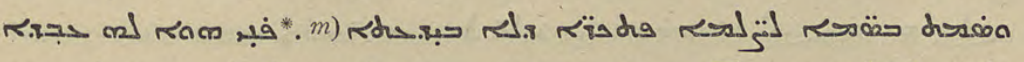

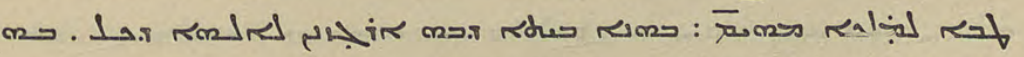

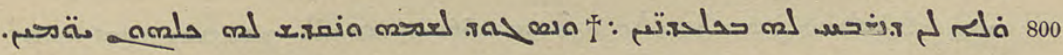

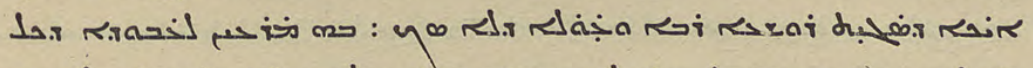

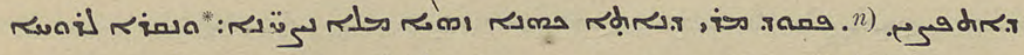

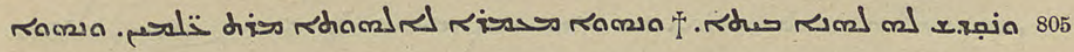

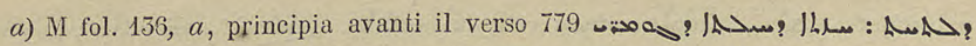

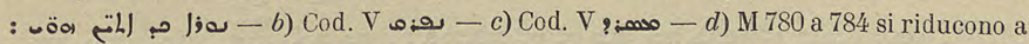

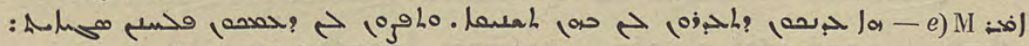

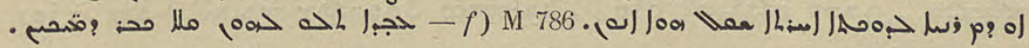

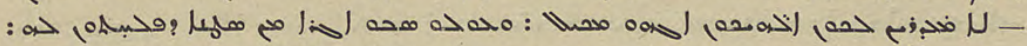

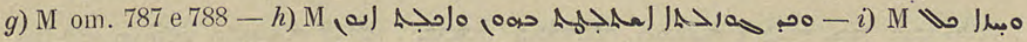

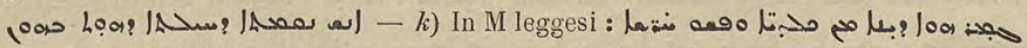

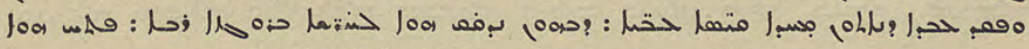

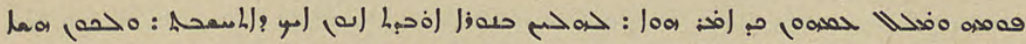

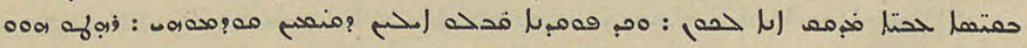

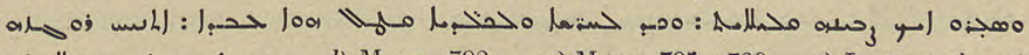
: l) M om. 792 - m) M om. 795 e 796 - n) Lacuna: devesi 


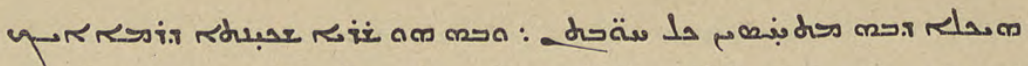

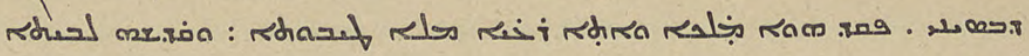

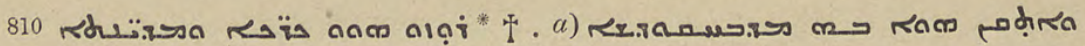

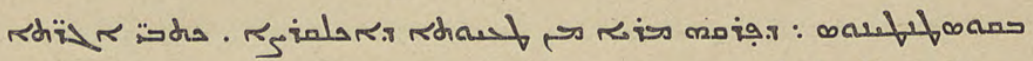

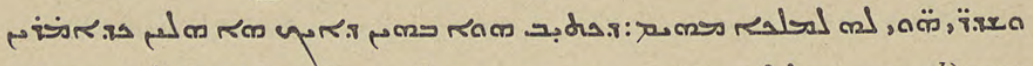

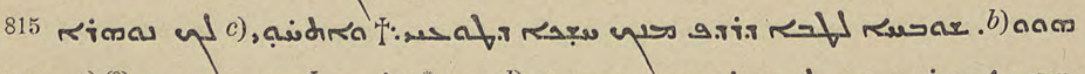

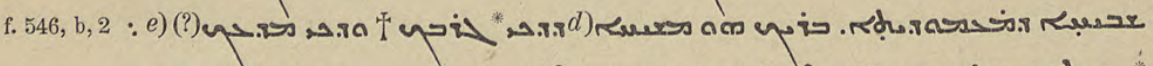

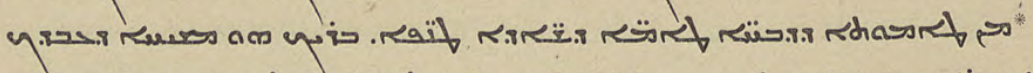

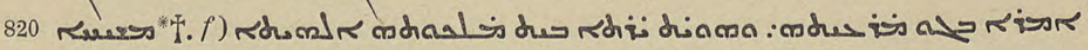

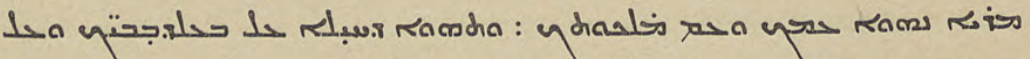

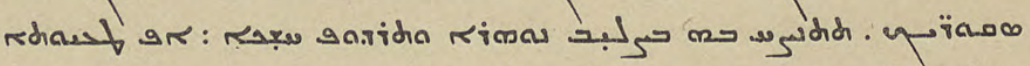

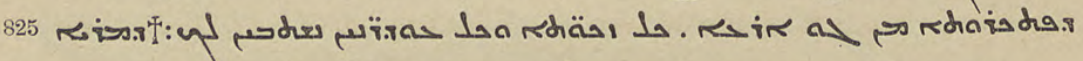

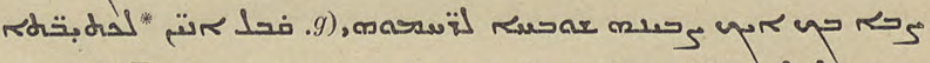
مصa

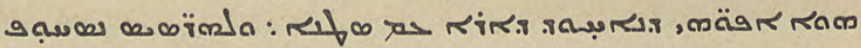

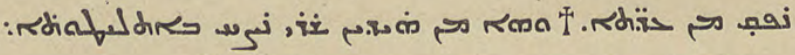

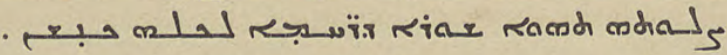
l) rals oasififoa to risks ple

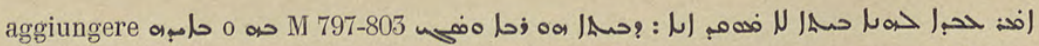

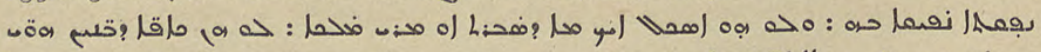

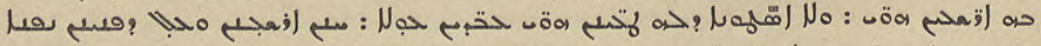

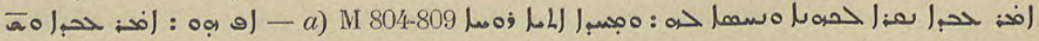

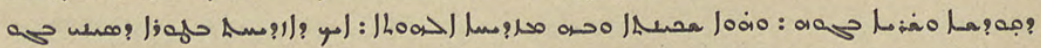

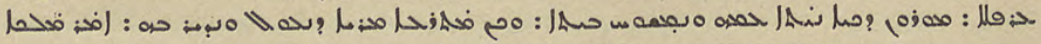

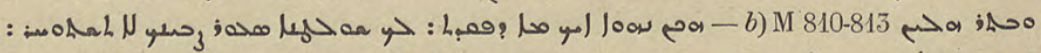

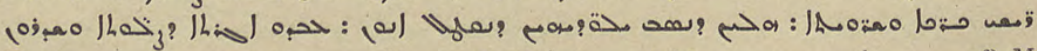

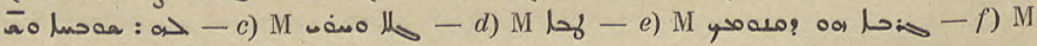
o90

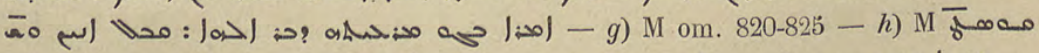

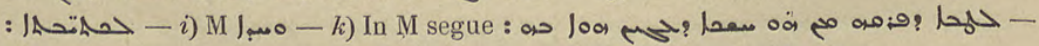

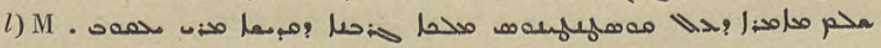

